\title{
Cytotoxicity and Bioactivity of Dental Pulp-Capping Agents towards Human Tooth-Pulp Cells: A Systematic Review of In-Vitro Studies and Meta-Analysis of Randomized and Controlled Clinical Trials
}

\author{
Mariano S. Pedano ${ }^{1,+}+\mathbb{C}$, Xin Li ${ }^{1, \dagger}$, Kumiko Yoshihara ${ }^{2,3}$, Kirsten Van Landuyt ${ }^{1}$ \\ and Bart Van Meerbeek $1, *$ (D) \\ 1 Department of Oral Health Sciences, KU Leuven (University of Leuven), BIOMAT-Biomaterials Research \\ Group \& UZ Leuven, University Hospitals Leuven, 3000 Leuven, Belgium; \\ simon.pedano@kuleuven.be (M.S.P.); xin.li@kuleuven.be (X.L.); kirsten.vanlanduyt@uzleuven.be (K.V.L.) \\ 2 National Institute of Advanced Industrial Science and Technology (AIST), Health Research Institute, \\ 2217-14 Hayashi-Cho, Takamaysu, Kagawa 761-0395, Japan; k-yoshi@md.okayama-u.ac.jp \\ 3 Department of Pathology \& Experimental Medicine, Dentistry and Pharmaceutical Sciences, \\ Graduate School of Medicine, Okayama University, 2-5-1 Shikata-cho, Kita-ku, Okayama 700-8558, Japan \\ * Correspondence: bart.vanmeerbeek@kuleuven.be \\ + These authors contributed equally to this work.
}

Received: 15 May 2020; Accepted: 8 June 2020; Published: 12 June 2020

\begin{abstract}
Background. In the era of biology-driven endodontics, vital pulp therapies are regaining popularity as a valid clinical option to postpone root-canal treatment. In this sense, many different materials are available in the market for pulp-capping purposes. Objectives. The main aim of this systematic review and meta-analysis was to examine literature regarding cytotoxicity and bioactivity of pulp-capping agents by exposure of human dental pulp cells of primary origin to these materials. A secondary objective was to evaluate the inflammatory reaction and reparative dentin-bridge formation induced by the different pulp-capping agents on human pulp tissue. Data sources. A literature search strategy was carried out on PubMed, EMBASE and the Web of Science databases. The last search was done on 1 May 2020. No filters or language restrictions were initially applied. Two researchers independently selected the studies and extracted the data. Study selection included eligibility criteria, participants and interventions, study appraisal and synthesis methods. In vitro studies were included when human dental pulp cells of primary origin were (in) directly exposed to pulp-capping agents. Parallel or split-mouth randomized or controlled clinical trials (RCT or CCT) were selected to investigate the effects of different pulp-capping agents on the inflammation and reparative bridge-formation capacity of human pulp tissue. Data were synthesized via odds ratios ( $95 \%$ confidence interval) with fixed or random effects models, depending on the homogeneity of the studies. The relative risks ( $95 \%$ confidence interval) were presented for the sake of interpretation. Results. In total, 26 in vitro and 30 in vivo studies were included in the systematic review and meta-analysis, respectively. The qualitative analysis of in vitro data suggested that resin-free hydraulic calcium-silicate cements promote cell viability and bioactivity towards human dental pulp cells better than resin-based calcium-silicate cements, glass ionomers and calcium-hydroxide cements. The meta-analysis of the in vivo studies indicated that calcium-hydroxide powder/saline promotes reparative bridge formation better than the popular commercial resin-free calcium-silicate cement Pro-Root MTA (Dentsply-Sirona), although the difference was borderline non-significant $(p=0.06)$, and better than calcium-hydroxide cements $(p<0.0001)$. Moreover, resin-free pulp-capping agents fostered the formation of a complete reparative bridge better than resin-based materials $(p<0.001)$. On the other hand, no difference was found among the different materials tested regarding
\end{abstract}


the inflammatory effect provoked at human pulp tissue. Conclusions. Calcium-hydroxide $(\mathrm{CH})$ powder and Pro-Root MTA (Dentsply-Sirona) have shown excellent biocompatibility in vitro and in vivo when tested on human cells and teeth. Their use after many years of research and clinical experience seems safe and proven for vital pulp therapy in healthy individuals, given that an aseptic environment (rubber dam isolation) is provided. Although in vitro evidence suggests that most modern hydraulic calcium-silicate cements promote bioactivity when exposed to human dental pulp cells, care should be taken when these new materials are clinically applied in patients, as small changes in their composition might have big consequences on their clinical efficacy. Key findings (clinical significance). Pure calcium-hydroxide powder/saline and the commercial resin-free hydraulic calcium-silicate cement Pro-Root MTA (Dentsply-Sirona) are the best options to provide a complete reparative bridge upon vital pulp therapy. Systematic review registration number. PROSPERO registration number: CRD42020164374.

Keywords: vital pulp therapy; biomaterials; calcium hydroxide; MTA; bioceramics; human dental pulp cells; hydraulic calcium-silicate cements

\section{Introduction}

Dental pulp-capping agents are defined as those materials used as a protective layer to an exposed tooth pulp to allow the tissue to recover and maintain its normal function and vitality $[1,2]$. Ideally, those materials should not only be inert, in the sense that they should not be toxic to the pulp cells, but they should be "bioactive" towards the tissues by stimulating migration, proliferation and osteogenic differentiation of the cells [3,4].

The tenets of minimal-invasive dentistry have caused a paradigm shift in the treatment of deep caries and vital pulp therapies. In this way, from total caries-excavation techniques, we have moved onto partial caries-excavation to avoid pulp exposures [5-8]. Something similar is happening in the treatment of reversible and irreversible pulpitis. Increasing evidence is showing that in the presence of strict aseptic conditions (rubber dam isolation) and with the aid of magnification, partial or full pulpotomy can serve as valid and less invasive alternatives to root-canal treatment [8-11]. This might have many advantages, since root-canal treatment is a more technically demanding and time-consuming treatment than (partial) pulpotomy [12].

Since the introduction of Pro-Root MTA (MTA; Dentsply-Sirona, Konstanz, Germany), the first hydraulic calcium-silicate cement developed, many other materials with similar compositions have been introduced into the market [13-15]. The main reasons for the increase of marketed materials are the good results obtained with MTA in terms of biocompatibility and long-term survival [16,17], and the need for materials with improved handling properties, lesser discoloration risks, better sealing abilities and reduced prices $[18,19]$. The latest developments in this search for improved dental pulp-capping agents are the resin-based calcium-silicate cements [20-22]. These materials possess enhanced handling properties by setting on command; they reduce the risk of discoloration; and, by optimizing the monomer composition, they may also adhere to tooth structure, by which improved sealing capacity can be expected [23-25]. Moreover, by adding resins to their composition, we may also better adhere them to resin composites and resin-modified glass ionomers, being put on top; reduce the treatment time; and reduce the risks of leakage and early filling loss [26]. However, the main drawback of this type of cement is the lack of biocompatibility of the monomers in contact with vital pulp tissue, which may hamper the formation of a complete hard tissue barrier at the exposed area [27-29]. Nevertheless, resins are not toxic by definition and many researchers are already working to develop biocompatible, naturally derived resin blends that may be suitable for biomedical applications [30-34]. These new type of photocurable resins are only prototypes but they have already been tested in in vitro and in vivo studies with promising results [35-37]. 
The Guide to Clinical Endodontics from the American Association of Endodontists recommends pulpotomy in permanent teeth only as an emergency or interim procedure until further root-canal treatment can be accomplished. However, recent randomized clinical trials have challenged this concept, as pulpotomy might be a successful treatment option for teeth with symptomatic irreversible pulpitis, even in cases with periapical involvement [11,38-42]. As this type of treatment is becoming more scientifically supported, a recent review and position statement article from the European Society for Endodontology [8] opened the door for this paradigm shift and clinicians are starting to perform such treatments [43-45]. In this way, the best available evidence suggests that the materials of choice for vital pulp therapy are calcium-hydroxide or MTA [46]. However, these materials have many side effects. The main problem of calcium hydroxide is its high solubility, which will create a gap between pulp tissue and final restorative material [47]. For MTA, the main drawbacks are: (1) risk of discoloration, (2) long-setting time and (3) difficult handling [19]. Therefore, recently introduced materials are gaining popularity among clinicians; for example, tricalcium-silicate cements such as Biodentine (Septodont, Saint-Maur-des-Fossés, France), and resin-based calcium-silicate cements, such as Theracal LC (Bisco, Schaumburg, IL, USA) and Biocal Cap (Harvard, Hoppegarten, Germany). However, very limited information is available regarding their biocompatibility when they are exposed to human dental pulp cells and tissue.

Recently, many reviews have been written about dental pulp-capping therapies and materials [48-51]. However, concerningly, this is the first systematic review aiming to compare all kinds of pulp-capping agents, including resin-based materials. Moreover, by using an indirect meta-analytical approach, we have tried to shed some light on the controversy that some studies found no difference in terms of (long-term) survival and reparative bridge formation between calcium-hydroxide materials and calcium-silicate cements $[46,52,53]$, while others showed that calcium-silicate cements improved the prognosis of vital-pulp therapies $[49,50]$.

Therefore, the aim of this study was to assess the in vitro biocompatibility of dental pulp-capping materials for vital pulp therapy when exposed to human dental pulp cells. As a secondary objective, in vivo studies were reviewed for inflammatory reaction and the presence of reparative dentin formation after direct exposure of the pulp tissue of completely developed permanent teeth to pulp-capping materials.

The null-hypotheses tested were (1) that there is no difference in in vitro biocompatibility for the different pulp-capping agents when exposed to human dental pulp cells; (2) that there is no difference in the short-term ( $<30$ days) inflammatory reaction caused by the materials tested in vivo; and (3) that there is no difference in complete hard-tissue bridge formation in vivo after 30 days among the different materials tested.

\section{Material and Methods}

\subsection{Protocol and Registration}

The methodology of this review was based on the PRISMA (Preferred Reporting Items for Systematic Reviews and Meta-Analyses) guidelines [54,55]. This review was registered at the PROSPERO database (number: CRD42020164374).

The protocol for this review was designed by the authors with the support of an expert librarian from the Biomedical Sciences group of KU Leuven.

\subsection{Eligibility Criteria}

The eligibility criteria were different depending on the type of study (i.e., in vitro vs. in vivo studies) and the specific characteristics (inclusion and exclusion criteria) for each type of study are shown in Table 1. 
Table 1. Eligibility criteria for in vitro and in vivo studies.

\begin{tabular}{|c|c|c|}
\hline \multicolumn{3}{|c|}{ Eligibility criteria for in vitro studies } \\
\hline Characteristics & Inclusion Criteria & Exclusion Criteria \\
\hline Publication year & Studies published from 1993 & Studies published before 1993 \\
\hline Language & English & Other languages different than English \\
\hline Population & $\begin{array}{l}\text { Human dental pulp cells from a } \\
\text { primary source } \\
\text { Cells from more than } 1 \text { patient }\end{array}$ & $\begin{array}{l}\text { Other type of oral/dental cells or cells not from } \\
\text { primary source (i.e.,: immortalized cells, commercial } \\
\text { cell lines, ... ) } \\
\text { Cells obtained from one single patient }\end{array}$ \\
\hline Tests performed & $\begin{array}{l}\text { At least } 2 \text { different tests were } \\
\text { performed }\end{array}$ & $\begin{array}{l}\text { Only } 1 \text { test was performed (i.e.,: cytotoxicity, } \\
\text { mineralization ability, ...) }\end{array}$ \\
\hline Materials tested & $\begin{array}{l}\text { Commercially available materials } \\
\text { Resin-based or resin-free } \\
\text { pulp-capping materials }\end{array}$ & $\begin{array}{l}\text { Experimental materials or modification of an } \\
\text { existing material } \\
\text { Materials for other purposes (sealers, fixation } \\
\text { cements, monomers, ... ) }\end{array}$ \\
\hline Analysis & $\begin{array}{l}\text { At least } 2 \text { different materials } \\
\text { compared between each other }\end{array}$ & Materials compared only to the control \\
\hline \multicolumn{3}{|c|}{ Eligibility criteria for in vivo studies } \\
\hline Publication year & Studies published from 1993 & Studies published before 1993 \\
\hline Language & English & Other languages different than English \\
\hline $\begin{array}{l}\text { Ethical } \\
\text { Committee }\end{array}$ & Mentioned & No ethical committee mentioned in the text \\
\hline Population & $\begin{array}{l}\text { Permanent teeth } \\
\text { Healthy teeth (free from caries } \\
\text { or infection) } \\
\text { Patients' age and amount of teeth } \\
\text { should be provided }\end{array}$ & $\begin{array}{l}\text { Primary teeth } \\
\text { Teeth exhibiting caries or periodontal disease } \\
\text { Sample size and age of the participants } \\
\text { not mentioned }\end{array}$ \\
\hline Materials tested & $\begin{array}{l}\text { Commercially available materials } \\
\text { Resin-based or resin-free } \\
\text { pulp-capping materials } \\
\text { Clear description and brand of the } \\
\text { materials used }\end{array}$ & $\begin{array}{l}\text { Experimental materials or modification of an } \\
\text { existing material } \\
\text { Materials for other purposes (sealers, fixation } \\
\text { cements, monomers, ... ) } \\
\text { No clear description and brand of the materials used }\end{array}$ \\
\hline Analysis & $\begin{array}{l}\text { Histology } \\
\text { Inflammation and hard-tissue } \\
\text { formation }\end{array}$ & $\begin{array}{l}\text { Studies where "only" clinical parameters } \\
\text { were evaluated } \\
\text { No bridge formation evaluated }\end{array}$ \\
\hline Type of study & $\begin{array}{l}\text { Randomized or controlled clinical } \\
\text { trials (RCT or CCT) }\end{array}$ & Other type of studies (case reports, case series, ... ) \\
\hline
\end{tabular}

\subsection{Information Sources}

A literature search was performed using the PubMed, EMBASE and Web of Science databases (Figures S1-S3). The first search was performed on 1 October 2019 and was updated for the last time on 1 May 2020. No filters were applied. After removing duplicates with Endnote X9 software (Clarivate Analytics), we chose studies starting from 1993, because it is the date when mineral trioxide aggregate was patented, and together with calcium hydroxide it is considered as gold-standard material for pulp-preserving procedures. Finally, a manual search was conducted from the reference lists of relevant review articles published in the last 5 years.

\subsection{Search Strategy}

The search strategy was designed by 2 reviewers (MSP and XL) in collaboration with an expert librarian. As the main purpose of the review was not to find a specific answer to a specific clinical question, we decided to conduct the literature search using the main terms of interest, instead of the classic PICO structure. The main terms of interest chosen were: (1) "Biocompatibility or pulp-tissue reaction"; (2) "Pulp-capping materials or agents"; and, (3) "(Human) dental pulp cells/tissue or (human) teeth." 
The complete search strategy used in the PubMed, EMBASE and Web of Science databases can be found in the Supplementary Information (Figures S1-S3).

\subsection{Study Selection and Data Collection Process}

Studies were selected and data collected by two independent investigators (MSP and XL) who revised the full list of articles and selected the papers that were potentially of interest, first by title and then by abstract screening. Later, texts were fully screened to identify the articles that met the inclusion criteria. In case of disagreement, differences were discussed until agreement was reached. Only articles published in the English language were chosen (Figure 1).

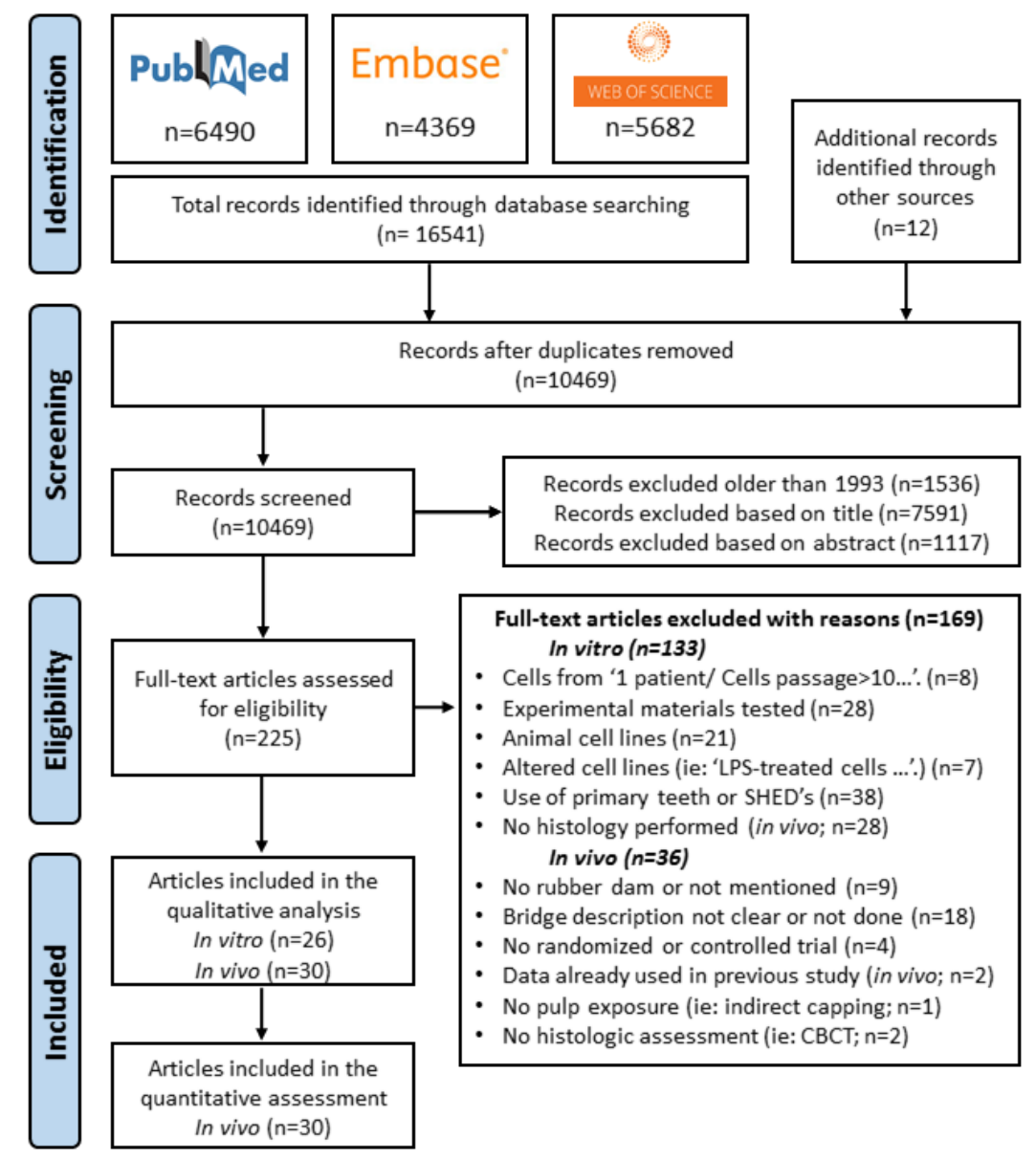

Figure 1. PRISMA flowchart describing the article screening procedure.

Data extraction was done separately for in vitro (Table 2) and in vivo studies (Tables 3 and 4). For the in vitro studies, the following data were obtained from the selected articles: (1) study characteristics: authors and year of publication, (2) materials tested, (3) type of exposure (direct/indirect) and the use of fresh or set materials, (4) parameters tested, (5) methods used and (6) results obtained. For the in vivo studies, the data collected were the following: (1) study characteristics: authors and year of publication, (2) type of study: randomized controlled trial (RCT) or controlled clinical trial (CCT), (3) method used for hemostasia, (4) materials tested, (5) etching of pulp tissue, (6) evaluation time, (7) presence (or not) of an independent examiner of the histological samples, (8) characteristics of the bridge formed, (9) characteristics of the inflammatory reaction and (10) amount and type of teeth used and age of the patients. When the data from the articles were unclear or could not be found, we contacted the authors by e-mail or ResearchGate. If no answer was received, the articles were excluded. 
Table 2. Included in vitro studies. Symbols of greater than $(>)$, less than $(<)$ or equal to $(=)$ are used to compare the results of the tested groups.

\begin{tabular}{|c|c|c|c|c|c|}
\hline Studies & Materials & Type of Exposure & Parameters & Methods & Results \\
\hline \multirow{5}{*}{$\begin{array}{l}\text { Alliot-Licht } \\
\text { et al. } \\
\text { (1994) [56] }\end{array}$} & \multirow{5}{*}{$\begin{array}{l}\text { Calcium hydroxide }(\mathrm{CH}) \\
\text { Hydroxyapatite (HAp) }\end{array}$} & \multirow{5}{*}{$\begin{array}{l}\mathrm{CH} \text { particles sterilized by } \\
\text { heating }\left(180^{\circ} \mathrm{C}-1 \mathrm{~h}\right) ; \\
\text { direct contact } \\
\text { (materials powder in } \\
\text { culture medium) }\end{array}$} & Cell morphology & Light microscopy (at $3 \& 5$ days) & $\begin{array}{c}\text { CH inhibited pulp fibroblasts growth (<cell density than } \\
\text { control; subjective observation) } \\
\text { HAp did not affect the cell density }(\approx \text { cell density as the } \\
\text { control; subjective observation) }\end{array}$ \\
\hline & & & Phagocytotic activity & TEM (at day 5) & $\begin{array}{l}\text { Close contact of } \mathrm{CH} \text { particles with fibroblasts' membrane } \\
\text { HAp particles were closely bound to cell membrane or } \\
\text { internalized by the cells. } \\
\text { Cells cultured in the presence of } \mathrm{CH} \text { exhibited ghost cells } \\
\text { and electron-dense spherical vesicles in the cytoplasm of } \\
\text { living cells. TEM revealed HAp particles within the cells }\end{array}$ \\
\hline & & & & DNA synthesis (at 1, 2, $3 \& 4$ days) & $\begin{array}{l}\mathrm{CH} \text { and HAp delayed the proliferation of cells at all } \\
\text { time points. }\end{array}$ \\
\hline & & & Cell proliferation & Protein synthetic activity (at 6 days) & $\begin{array}{c}\mathrm{CH}<\text { incorporation of }\left[{ }^{3} \mathrm{H}\right] \text {-leucine and }\left[{ }^{3} \mathrm{H}\right] \text {-proline by } \\
\text { pulp fibroblasts at day } 6 . \\
\text { HAp }>\text { incorporation of }\left[{ }^{3} \mathrm{H}\right] \text {-leucine and }\left[{ }^{3} \mathrm{H}\right] \text {-proline by } \\
\text { the pulp fibroblasts at day } 6 .\end{array}$ \\
\hline & & & Cell differentiation & $\begin{array}{l}\text { ALP activity (at } 8 \text { days for } \mathrm{CH} \text {; at } 5 \\
\qquad \& 8 \text { days for HAp) }\end{array}$ & $\begin{array}{l}\text { CH inhibited ALP activity of pulp fibroblasts at day } 8 \text {. } \\
\text { HAp inhibited ALP activity of pulp fibroblasts at } 5 \text { and } \\
8 \text { days. }\end{array}$ \\
\hline \multirow{3}{*}{ Min et al. (2007) [57] } & \multirow{3}{*}{$\begin{array}{l}\text { Portland cement (PC) } \\
\text { Portland cement with } \\
\text { bismuth oxide (BPC) }\end{array}$} & \multirow{3}{*}{$\begin{array}{l}\text { Indirect contact } \\
\text { (SET materials) }\end{array}$} & Cell viability & MTT assay (at $12,24,48 \& 72 \mathrm{~h}$ ) & $\begin{aligned} \mathrm{PC} & >\mathrm{BPC} \text { at } 12 \text { and } 24 \mathrm{~h} \\
\mathrm{PC} \approx \mathrm{BPC} & \end{aligned}$ \\
\hline & & & Nitric oxide production & Griess reaction(at $12,24,48 \& 72 \mathrm{~h}$ ) & $\begin{array}{l}\mathrm{BPC}>\text { nitrite production than } \mathrm{PC} \text { at } 12 \text { and } 24 \mathrm{~h} . \\
\mathrm{PC} \approx \mathrm{BPC} \text { nitrite production at } 48 \text { and } 72 \mathrm{~h} .\end{array}$ \\
\hline & & & Ho-1 and iNOS & RT-PCR (at 12, 24, 48 \& 72 h) & $\begin{array}{l}\text { Ho-1: } \mathrm{PC}<\mathrm{BPC} \text { at all study periods } \\
\text { iNOS: } \mathrm{PC}<\mathrm{BPC} \text { at all study points }\end{array}$ \\
\hline \multirow{3}{*}{ Min et al. (2007) [58] } & \multirow{3}{*}{$\begin{array}{l}\text { Portland cement (PC) } \\
\text { Fuji-II LC (Fuji-II, GC) } \\
\text { Zinc-oxide Eugenol (IRM; } \\
\text { Dentsply-Sirona) } \\
\text { CH cement Dycal } \\
\text { (Dentsply-Sirona) }\end{array}$} & \multirow{3}{*}{$\begin{array}{l}\text { Direct and indirect contact } \\
\text { (SET materials) }\end{array}$} & Cell morphology & SEM (at $24 \mathrm{~h})$ & $\begin{array}{l}\text { PC: showed flattened cells close to one another and } \\
\text { spreading across the substrate. } \\
\text { Fuji-II, IRM, and Dycal: no living cells were seen. }\end{array}$ \\
\hline & & & Cell viability & MTT assay (at $12,24,48 \& 72 \mathrm{~h}$ ) & $\begin{array}{c}\text { PC } \approx \text { control at all study periods. } \\
\text { PC > Fuji-II, Dycal and IRM at all study periods. } \\
\text { Control > Fuji II LC, IRM, and Dycal at all study points. }\end{array}$ \\
\hline & & & Cell differentiation & RT-PCR (ON, DSPP) (at 7 days) & $\begin{array}{l}\text { ON: PC } \approx \text { positive control group. } \\
\text { DSPP: PC stimulated mineralization but less than the } \\
\text { positive control. }\end{array}$ \\
\hline
\end{tabular}


Table 2. Cont

\begin{tabular}{|c|c|c|c|c|c|}
\hline Studies & Materials & Type of Exposure & Parameters & Methods & Results \\
\hline \multirow{3}{*}{$\begin{array}{l}\text { Laurent } \\
\text { et al. (2008) [59] }\end{array}$} & \multirow{3}{*}{$\begin{array}{l}\mathrm{Ca}_{3} \mathrm{SiO}_{-5} \text { cement (CS) } \\
\text { Dycal (Dentsply-Sirona) } \\
\text { Pro-Root MTA (MTA; } \\
\text { Dentsply-Sirona) }\end{array}$} & \multirow{3}{*}{$\begin{array}{l}\text { Indirect contact* } \\
\text { (SET materials) } \\
\text { [ISO-Standard-(Nr. } \\
\text { Not mentioned)] }\end{array}$} & Cell viability & MTT assay (at $24 \mathrm{~h}$ ) & $\begin{array}{l}\text { No contact (disk diffusion): } \mathrm{CS} \approx \mathrm{MTA} \approx \mathrm{Dyc} \\
\text { Indirect contact (eluates from materials): } \\
\mathrm{Cs} \approx \mathrm{MTA}>\text { Dycal }\end{array}$ \\
\hline & & & Cell differentiation & $\begin{array}{l}\text { Immunohistochemistry (at } \\
\quad 4 \text { weeks) }\end{array}$ & $\begin{array}{l}\text { MTA and CS expressed Nestin and Collagen I at a similar } \\
\text { level as the control group. Both materials generated } \\
\text { mineral deposits at a similar level as the control group. }\end{array}$ \\
\hline & & & Genotoxicity & $\begin{array}{l}\text { Micronuclei test } \\
\text { Comet assay }\end{array}$ & $\begin{array}{l}\text { CS does not induce reverse mutations with/without the } 59 \\
\text { metabolic activation system. } \\
\text { CS generated lymphocytes with micronuclei } \approx \text { as the } \\
\text { negative control. } \\
\text { CS generated DNA in the tail } \approx \text { as the negative control } \\
\text { and }<\text { than the cytotoxic control. }\end{array}$ \\
\hline \multirow{3}{*}{ Min et al. (2009) [60] } & \multirow{3}{*}{$\begin{array}{l}\text { Radiopaque Portland } \\
\text { cement (RPC) } \\
\text { Portland cement (PC) IRM } \\
\text { (Dentsply-Sirona) }\end{array}$} & \multirow{3}{*}{$\begin{array}{l}\text { Direct and indirect contact } \\
\text { (SET materials) }\end{array}$} & Cell morphology & SEM (at $48 \mathrm{~h}$ ) & $\begin{array}{l}\text { PC and RPC: Spread and flattened HDPCs. The density } \\
\text { and characteristics of the HDPCs in both groups were } \\
\text { similar to that on control samples. } \\
\text { IRM: no living cells were seen in contact with the }\end{array}$ \\
\hline & & & \multirow[b]{2}{*}{ Cell differentiation } & $\begin{array}{c}\text { ALP activity } \\
\text { (at } 1,3,7 \& 14 \text { days) } \\
\text { ARS staining }(1,2 \& 3 \mathrm{wk})\end{array}$ & $\begin{array}{l}\text { 1d: PC and RPC > control; 3d, 7d, 14d: control > PC } \\
\text { and RPC }\end{array}$ \\
\hline & & & & $\begin{array}{l}\text { AKs staining }(1, \angle \& 3 \text { WK) } \\
\text { RT-PCR (DSPP, ON) } \\
\text { (at } 1,3,7 \text { \& } 14 \text { days) }\end{array}$ & $\begin{array}{l}\text { 2wk and } 3 w k \text { : PC and PCR }>\text { control } \\
\text { DSPP: PC and RPC }>\text { control at day } 14 ; \text { OCN: control } \approx \mathrm{PC} \\
\text { and RPC at all study periods. }\end{array}$ \\
\hline \multirow[b]{3}{*}{ Lee et al. (2014) [61] } & \multirow{3}{*}{$\begin{array}{c}\text { ProRoot MTA (MTA; } \\
\text { Dentsply-Sirona) } \\
\alpha \text {-tricalcium } \\
\text { phosphate-based cement } \\
\quad(\alpha-\mathrm{TCP})\end{array}$} & \multirow[b]{3}{*}{$\begin{array}{l}\text { Direct and indirect contact } \\
\text { (SET materials) }\end{array}$} & Cell morphology & SEM (at $72 \mathrm{~h}$ ) & $\begin{array}{l}\text { hDPCs in contact with MTA and } \alpha \text {-TCP were well-spread } \\
\text { and flattened. }\end{array}$ \\
\hline & & & Cell viability & MTT assay (at 1, 2, 3, $7 \& 14$ days) & $\begin{array}{l}\text { MTA and } \alpha-\mathrm{TCP} \approx \text { control until day } 7 \\
\alpha-\mathrm{TCP}>\mathrm{MTA} \text { at } 14 \mathrm{~d} ; \alpha-\mathrm{TCP} \approx \text { control }\end{array}$ \\
\hline & & & Cell differentiation & $\begin{array}{l}\text { Western blot (DSPP, DMP- } 1 \text { and } \\
\text { ON) (at } 3 \text { days) } \\
\text { ARS staining (at } 14 \text { days) } \\
\text { Immunofluorescence (DSPP, } \\
\text { DMP- } 1 \text { and ON) (at } 7 \text { days) }\end{array}$ & $\begin{array}{c}\alpha-\mathrm{TCP} \approx \text { MTA for DSPP, DMP- } 1 \text { and ON. } \\
\alpha-\mathrm{TCP} \approx \text { MTA for DSPP, DMP- } 1 \text { and ON. } \\
\alpha-\mathrm{TCP} \text { and MTA induced higher protein signals than the } \\
\text { control group. }\end{array}$ \\
\hline
\end{tabular}


Table 2. Cont.

\begin{tabular}{|c|c|c|c|c|c|}
\hline Studies & Materials & Type of Exposure & Parameters & Methods & Results \\
\hline \multirow[t]{2}{*}{$\begin{array}{l}\text { Bortoluzzi et al. } \\
\text { (2015) [62] }\end{array}$} & \multirow[t]{2}{*}{$\begin{array}{l}\text { Biodentine }(B d ; \\
\text { Septodont) } \\
\text { Theracal LC (Th; Bisco) } \\
\text { MTA Angelus } \\
\text { (MTA-A; Angelus) }\end{array}$} & \multirow[t]{2}{*}{$\begin{array}{l}\text { Indirect contact* } \\
\text { (SET materials) }\end{array}$} & Cell viability & $\begin{array}{l}\text { XTT assay (direct and indirect } \\
\text { eluate evaluations) } \\
\text { Flow cytometry- } \\
\text { Annexin V-PI } \\
\text { (4 weekly cycles) }\end{array}$ & $\begin{array}{c}\text { Direct evaluation: } \\
\text { 1st cycle: control }>\mathrm{Bd}>\text { MTA-A and Th } \\
\text { 2nd cycle: control }>\mathrm{Bd} \approx \mathrm{MTA}-\mathrm{A}>\mathrm{Th} \\
\text { 3rd cycle: control } \geq \mathrm{MTA}-\mathrm{A} \geq \mathrm{Bd}>\mathrm{Th} \\
\text { 4th cycle: control } \approx \mathrm{Bd} \approx \mathrm{MTA}-\mathrm{A}>\mathrm{Th} \\
\text { Indirect eluate evaluation: } \\
\text { 1:1\&1:10 dilutions: control }>\text { MTA-A } \approx \mathrm{Bd}>\mathrm{Th} ; 1: 100 \\
\text { dilution: control } \approx \text { MTA-A } \approx \mathrm{Bd}>\mathrm{Th} \\
\text { Percentage of healthy, non-apoptotic and non-necrotic } \\
\text { cells: control }>\text { MTA-A } \approx \mathrm{Bd}>\text { Th } \\
\text { Th was the most cytotoxic material causing apoptosis } \\
\text { and necrosis. }\end{array}$ \\
\hline & & & Cell differentiation & $\begin{array}{l}\text { qRT-PCR (DSPP, OCN, BSP, RUNX } \\
\text { 2, DMP- } 1 \text { and ALP) (at } 7 \text { days) } \\
\text { ALP activity (at } 14 \text { days) } \\
\text { ARS and TEM (at } 21 \text { days) }\end{array}$ & $\begin{array}{c}\text { ALP; OCN; BSP; DSPP; DMP-1: Bd and } \\
\text { MTA-A > control } \approx \text { Th } \\
\text { RUNX 2: Bd } \approx \text { MTA-A } \approx \text { control } \approx \text { Th } \\
\text { Bd } \approx \text { control }>\text { MTA-A }>\text { Th } \\
\text { Bd }>\text { control }>\text { MTA-A }>\text { Th }\end{array}$ \\
\hline \multirow[t]{2}{*}{$\begin{array}{l}\text { Niu et al. } \\
\text { (2015) [63] }\end{array}$} & \multirow[t]{2}{*}{$\begin{array}{l}\text { ProRoot MTA (MTA; } \\
\text { Dentsply-Sirona) } \\
\text { Quick-Set2 (Qs; Avalon } \\
\text { Biomed Inc) }\end{array}$} & \multirow[t]{2}{*}{$\begin{array}{l}\text { Direct and indirect contact } \\
\text { (SET materials) }\end{array}$} & Cell viability & $\begin{array}{c}\text { Flow cytometry- } \\
\text { Annexin V-PI } \\
\text { ( } 3 \text { weekly cycles) } \\
\text { Leakage of cytosolic enzyme } \\
\text { ( } 3 \text { weekly cycles) } \\
\text { Caspase- } 3 \text { acitivity } \\
\text { ( } 3 \text { weekly cycles) } \\
\text { Oxidative stress ( } 3 \text { weekly cycles) }\end{array}$ & $\begin{array}{c}\text { Number of healthy cells: } \\
\text { 1st cycle: control }>\text { Qs }>\text { MTA }(p<0.001)>\text { IRM } \\
\text { 2nd cycle: control }>\text { Qs } \approx \text { MTA }>\text { IRM; 3rd cycle: control } \\
\geqslant \text { MTA } \geqslant \text { Qs }>\text { IRM } \\
\text { Percentage of cytotoxicity: } \\
\text { 1st cycle: } \text { IRM }>\text { MTA }>\text { Qs }>\text { control; } 2 \text { nd and 3rd cycles: } \\
\text { IRM }>\text { MTA } \approx \text { Qs }>\text { control } \\
\text { Relative caspase-3 activity: } \\
\text { 1st cycle: } \text { IRM }>\text { MTA }>\text { Qs }>\text { control; } 2 \text { nd cycle: IRM }> \\
\text { MTA }>\text { Qs }>\text { control } \\
\text { 3rd cycle: IRM }>\text { MTA } \approx \text { Qs }>\text { control } \\
\text { Oxidative stress: } \\
\text { 1st cycle: } \text { IRM }>\text { MTA }>\text { Qs }>\text { control; } 2 \text { nd cycle: IRM }> \\
\text { MTA } \approx \text { Qs }>\text { control } \\
\text { 3rd cycle: IRM }>\text { MTA } \approx \text { Qs } \approx \text { control }\end{array}$ \\
\hline & & & Cell proliferation & $\begin{array}{l}\text { MTT assay ( } 3 \text { weekly } \\
\text { cycles)Cellular DNA content } \\
\text { (3 weekly cycles) }\end{array}$ & $\begin{array}{c}\text { 1st cycle: control }>\text { Qs }>\text { MTA }>\text { IRM } \\
\text { 2nd cycle: control }>\text { Qs } \approx \text { MTA }>\text { IRM } \\
\text { 3rd cycle: control }>\text { Qs }>\text { MTA }>\text { IRM } \\
\text { DNA content: } \\
\text { 1st cycle: control }>\text { Qs }>\text { MTA }>\text { IRM } \\
\text { 2nd cycle: control }>\text { Qs } \approx \text { MTA }>\text { IRM } \\
\text { 3rd cycle: control }>\text { Qs } \approx \text { MTA }>\text { IRM }\end{array}$ \\
\hline
\end{tabular}


Table 2. Cont

\begin{tabular}{|c|c|c|c|c|c|}
\hline Studies & Materials & Type of Exposure & Parameters & Methods & Results \\
\hline & & & Cell viability & $\begin{array}{c}\text { XTT assay } \\
(24,48 \& 72 \mathrm{~h})\end{array}$ & $\begin{array}{c}24 \mathrm{~h} ; 1: 1 \text { and } 1: 2 \text { dilutions: } \mathrm{iBP}>\mathrm{W}-\mathrm{MTA}-\mathrm{A} ; 1: 5 \text { and } 1: 10 \\
\text { dilutions: } \mathrm{BPP} \approx \mathrm{MTA}-\mathrm{A} \\
48 \mathrm{~h} ; 1: 1 \text { dilution: } \mathrm{iBP}>\mathrm{W}-\mathrm{MTA}-\mathrm{A} ; 1: 2,1: 5 \text { and } 1: 10 \\
\text { dilutions: } \mathrm{BPP} \approx \mathrm{MTA}-\mathrm{A} \\
72 \mathrm{~h} \text {; all concentrations: } \mathrm{BBP} \approx \mathrm{MTA}-\mathrm{A}\end{array}$ \\
\hline $\begin{array}{l}\text { Öncel Torun et al. } \\
\text { (2015) [64] }\end{array}$ & $\begin{array}{l}\text { iRoot BP Plus (iBP; } \\
\text { Innovative Bioceramix) } \\
\text { White MTA Angelus } \\
\text { (MTA-A; Angelus) }\end{array}$ & $\begin{array}{l}\text { Indirect contact } \\
\text { (SET materials) }\end{array}$ & Cell differentiation & $\begin{array}{c}\text { qRT-PCR } \\
\text { (BMP-2, ON, BSP, OPN, DSPP, Col I } \\
\text { A1, HO- } 1 \text { at } 24 \& 72 \text { h) }\end{array}$ & 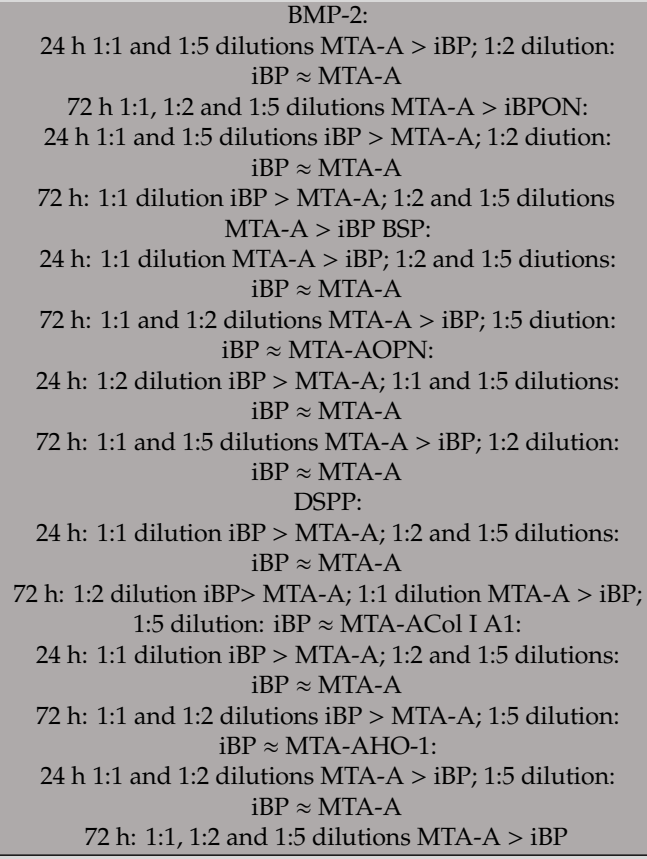 \\
\hline \multirow[b]{3}{*}{ Zhang et al.(2015) [65] } & \multirow[b]{3}{*}{$\begin{array}{l}\text { iRoot BP Plus (iBP; } \\
\text { Innovative Bioceramix) } \\
\text { ProRoot MTA (MTA; } \\
\text { Dentsply-Sirona) }\end{array}$} & \multirow[b]{3}{*}{$\begin{array}{l}\text { Indirect contact (SET } \\
\text { materials) } \\
\text { (ISO 10993-5) }\end{array}$} & Cell Viability & $\begin{array}{l}\text { Flow cytometry- } \\
\text { Annexin V-PI }\end{array}$ & $\mathrm{iBP} \approx \mathrm{MTA} \approx$ control \\
\hline & & & Cell Migration & $\begin{array}{l}\text { Wound-healing at } 24 \mathrm{~h} \\
\text { Transwell assay at } 24 \mathrm{~h}\end{array}$ & $\begin{array}{l}\mathrm{iBP} \approx \mathrm{MTA}>\text { control } \\
\mathrm{iBP}=\mathrm{MTA}>\text { control }\end{array}$ \\
\hline & & & $\begin{array}{l}\text { Cellular adhesion } \\
\text { and motility }\end{array}$ & $\begin{array}{c}\text { Western-Blot } \\
\text { (at } 5,10,30 \& 60 \mathrm{~min}) \\
\text { Cell Immunofluorescence assay } \\
\text { (at } 1 \mathrm{~h} \text { ) }\end{array}$ & $\begin{array}{c}\text { iBP led to phosphorylation of p38 MAPK, ERK 1/2, JNK, } \\
\text { Akt, and FGFR } \\
\text { iBP significantly increased p-focal adhesion kinase } \\
\text { (p-FAK), p-paxillin, and vinculin } \\
\text { Cells treated with iBP showed highly organized and } \\
\text { stretched stress fiber assembly }\end{array}$ \\
\hline
\end{tabular}


Table 2. Cont

\begin{tabular}{|c|c|c|c|c|c|}
\hline Studies & Materials & Type of Exposure & Parameters & Methods & Results \\
\hline \multirow{3}{*}{$\begin{array}{l}\text { Chung CJ et al. } \\
\text { (2016) [66] }\end{array}$} & \multirow{3}{*}{$\begin{array}{c}\text { Dycal (Dy; } \\
\text { Dentsply-Sirona) } \\
\text { Endocem Zr (E-Zr, } \\
\text { Maruchi) } \\
\text { White ProRoot MTA } \\
\text { (MTA; Dentsply-Sirona) } \\
\text { Retro-MTA (R-MTA; } \\
\text { Bio MTA) }\end{array}$} & \multirow{3}{*}{$\begin{array}{l}\text { Indirect and direct contact; } \\
\text { SET (s) and FRESH (f) } \\
\text { materials }\end{array}$} & $\begin{array}{c}\text { Cell } \\
\text { morphology/attachment }\end{array}$ & $\begin{array}{l}\text { Phase microscopy } \\
\text { (at } 3 \& 7 \text { days) } \\
\text { SEM (at } 3 \& 7 \text { days) }\end{array}$ & $\begin{array}{c}\text { 3d: MTA > cell morphology and attachement than } \\
\text { R-MTA and E-Zr } \\
\text { 7d: MTA, R-MTA and E-Zr sowed good cell morphology } \\
\text { and attachement } \\
\text { Dycal treated cells were dead after } 3 \text { and } 7 \text { days. Dycal } \\
\text { was not further used }\end{array}$ \\
\hline & & & Cell viability & XTT assay (at $3 \& 7$ days) & $\begin{array}{c}3 \mathrm{~d} \text { : control } \approx \text { MTA }(\mathrm{s}) \approx \mathrm{MTA}(\mathrm{f})>\mathrm{R}-\mathrm{MTA}(\mathrm{s}) \approx \mathrm{R}-\mathrm{MTA} \\
(\mathrm{f})>\mathrm{E}-\mathrm{Zr}(\mathrm{s}) \approx \mathrm{E}-\mathrm{Zr}(\mathrm{f}) \\
7 \mathrm{~d}: \operatorname{MTA}(\mathrm{f})>\operatorname{control} \approx \operatorname{MTA}(\mathrm{s}) \approx \mathrm{R}-\mathrm{MTA}(\mathrm{s}) \approx \mathrm{R}-\mathrm{MTA}(\mathrm{f}) \\
\approx \mathrm{E}-\mathrm{Zr}(\mathrm{f})>\mathrm{E}-\mathrm{Zr}(\mathrm{s})\end{array}$ \\
\hline & & & Angiogenic properties & $\begin{array}{l}\text { ELISA (VEGF, angiogenin, FGF-2) } \\
\text { (at } 3 \text { \& } 7 \text { days) }\end{array}$ & 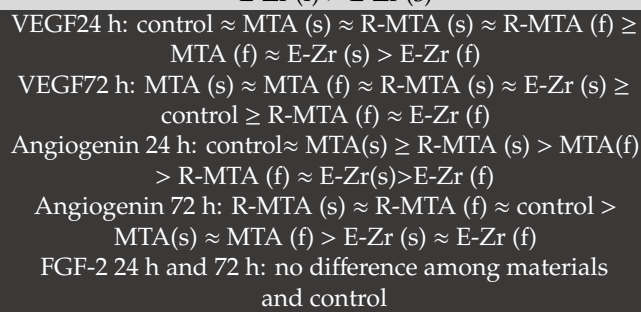 \\
\hline \multirow{2}{*}{$\begin{array}{l}\text { Daltoé M et al. } \\
\text { (2016) [67] }\end{array}$} & \multirow{2}{*}{$\begin{array}{c}\text { Biodentine (Bd; } \\
\text { Septodont) } \\
\text { White ProRoot MTA } \\
\text { (MTA; Dentsply-Sirona) }\end{array}$} & \multirow{2}{*}{$\begin{array}{l}\text { Indirect contact } \\
\text { (SET materials) } \\
\text { (ISO 10993-5) }\end{array}$} & Cell Viability & MTT assay (at $24 \& 48$ h) & $\begin{array}{c}24 \mathrm{~h}: \text { control } \approx \text { MTA_1:100 } \approx \text { Bd_1:100 > MTA_1:10, } \\
\text { Bd_1:10, Bd_1:1 and MTA_1:1 } \\
48 \mathrm{~h}: \text { control_ } \approx \text { MTA_1:100 } \approx \text { Bd_1:100 > MTA_1:10, } \\
\text { Bd_1:10, MTA_1:1 and Bd_1:1 }\end{array}$ \\
\hline & & & Cell differentiation & $\begin{array}{l}\text { qRT-PCR (SPP1, IBSP, DSPP, ALP 1, } \\
\text { DMP-1 and RUNX } 2 \text { (at } 24 \& 48 \text { h) }\end{array}$ & $\begin{array}{l}\text { SPP1 \& ALP1 \& RUNX2 at } 24 \mathrm{~h}: \text { Bd and MTA } \approx \text { control } \\
\text { SPP1 \& ALP1 \& RUNX2 } 48 \mathrm{~h} \text { : Bd and MTA > control } \\
\text { IBSP \& DSPP \& DMP1: } 24 \mathrm{~h} \text { and } 48 \mathrm{~h}: \text { no expression }\end{array}$ \\
\hline \multirow{4}{*}{$\begin{array}{l}\text { Widbiller M et al. } \\
\text { (2016) [68] }\end{array}$} & \multirow{4}{*}{$\begin{array}{l}\text { Biodentine (Bd; } \\
\text { Septodont) } \\
\text { GI Ketac-Molar (KM; 3M) } \\
\text { ProRoot MTA (MTA; } \\
\text { Dentsply-Sirona) }\end{array}$} & \multirow{4}{*}{$\begin{array}{l}\text { Indirect and direct contact } \\
\text { (SET materials) }\end{array}$} & $\begin{array}{c}\text { Cell } \\
\begin{array}{c}\text { morphology/attachement } \\
\text { (only Bd) }\end{array}\end{array}$ & SEM (at $24 \mathrm{~h}$ ) & $\begin{array}{l}\text { Biodentine: cells showed adhesion to and spreading onto } \\
\text { the cement surface } \\
{ }^{*} \text { Not done for the other materials. }\end{array}$ \\
\hline & & & Cell viability & $\begin{array}{c}\text { MTT assay (at } 1,3,5,7,10 \& \\
14 \text { days) }\end{array}$ & $\begin{array}{c}\text { Bd }>\text { other materials and control at } 1,3,5 \text { and } 7 \mathrm{~d} ; \\
\text { Bd } \approx \text { MTA }>\text { control }>\text { KM at } 10 \text { and } 14 \mathrm{~d} \\
\text { MTA }<\text { viability than the control at } 1 \mathrm{~d} ; \mathrm{MTA} \approx \text { cell } \\
\text { viability as the control at } 3-5-7 \mathrm{~d} ; \\
\mathrm{KM}<\text { cell viability than all the materials tested and the } \\
\text { control at all time points }\end{array}$ \\
\hline & & & $\begin{array}{l}\text { Cell differentiation } \\
\text { Not performed on KM }\end{array}$ & $\begin{array}{l}\text { RT-qPCR (ALP, Col-I A1, DSPP, } \\
\text { RUNX 2) (at } 7,14 \& 21 \text { days) }\end{array}$ & $\begin{array}{l}\text { Col-I A1 \& ALP: upregulated at 7d, especially for MTA, } \\
\text { and decreased steadily until 21d } \\
\text { DSPP: upregulated for MTA and BD at } 14 \text { and 21d } \\
\text { RUNX2: downregulated for MTA and BF throughout the } \\
\text { whole study period }\end{array}$ \\
\hline & & & & ALP activity (at 3, $7 \& 14$ days) & $\begin{array}{l}\text { ALP activity was downregulated for Bd at all times: } \\
\text { MTA } \approx \text { control }>\text { Bd }\end{array}$ \\
\hline
\end{tabular}


Table 2. Cont

\begin{tabular}{|c|c|c|c|c|c|}
\hline Studies & Materials & Type of Exposure & Parameters & Methods & Results \\
\hline \multirow{2}{*}{$\begin{array}{l}\text { Jeanneau C et al. } \\
\text { (2017) [28] }\end{array}$} & \multirow{2}{*}{$\begin{array}{c}\text { Biodentine (Bd; } \\
\text { Septodont) } \\
\text { Theracal LC (Th; Bisco) } \\
\text { Xeno III (Dentsply-Sirona) }\end{array}$} & \multirow[t]{2}{*}{$\begin{array}{l}\text { Indirect contact } \\
\text { (SET materials) }\end{array}$} & Cell differentiation & $\begin{array}{l}\text { Immunofluorescence } \\
\text { (DSP and Nestin at day 7) }\end{array}$ & $\begin{array}{r}\text { Bd_0.05 } \mathrm{cm}^{2} / \mathrm{mL}>\text { Th_ } 0.05 \mathrm{~cm}^{2} / \mathrm{mL}(p<0.05) \approx \text { control at } \\
3,5 \text { and } 7 \text { days } \\
\text { Bd_0.5 } \mathrm{cm}^{2} / \mathrm{mL}>\text { Th_ } 0.5 \mathrm{~cm}^{2} / \mathrm{mL}(p<0.05) \approx \text { control at } 3, \\
5 \text { and } 7 \text { days } \\
\text { Bd increased the expression of both markers, while Th } \\
\text { had no effect }\end{array}$ \\
\hline & & & Inflammatory effect & ELISA (IL-8; 24 and 48 h) & 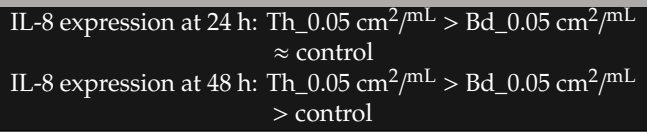 \\
\hline \multirow[t]{2}{*}{ Jun S-K et al. (2017) [69] } & \multirow{2}{*}{$\begin{array}{l}\text { Activa Bioactive (Activa; } \\
\text { Pulpdent) } \\
\text { Dycal (Dy; } \\
\text { Dentsply-Sirona) } \\
\text { Theracal LC (Th; Bisco) }\end{array}$} & \multirow[t]{2}{*}{$\begin{array}{l}\text { Indirect contact (SET } \\
\text { materials) } \\
\text { (ISO 10993-12) }\end{array}$} & Cell viability & $\begin{array}{c}\text { MTS assay (at } 24 \mathrm{~h} \text { ) } \\
\text { Live/dead assay (at } 24 \mathrm{~h} \text { ) }\end{array}$ & $\begin{array}{c}\text { 3.125\% eluates: Dy }>\mathrm{Th}>\text { Activa } \approx \text { control; } 6.25 \% \text { eluates: } \\
\text { Dy }>\mathrm{Th} \approx \text { Activa } \approx \text { control } \\
12.5 \% \text { eluates: Dy } \approx \mathrm{Th} \approx \text { Activa } \approx \text { control; } 25 \% \text { eluates: } \\
\text { Dy }<\text { Activa }<\text { Th }<\text { control } \\
50 \% \text { eluates: } \text { Dy } \approx \text { Activa }<\text { Th }<\text { control } \\
50 \% \text { eluates: } \text { Dy }<\text { Activa }<\text { Th }<\text { control }\end{array}$ \\
\hline & & & Cell differentiation & $\begin{array}{l}\text { ALP (at days } 14 \text { and 21) } \\
\text { ARS (at } 21 \text { days) }\end{array}$ & $\begin{array}{l}14 \mathrm{~d}: \text { Th }>\text { Dy }>\text { Activa } \approx \text { Osteogenic medium } \\
21 \mathrm{~d}: \text { Th } \approx \text { Dy }>\text { Activa }>\text { Osteogenic medium } \\
\text { Th } \approx \text { Dy }>\text { Activa } \approx \text { Osteogenic medium }\end{array}$ \\
\hline \multirow[b]{2}{*}{ Lee B-N et al. (2017) [70] } & \multirow[b]{2}{*}{$\begin{array}{l}\text { ProRoot MTA (MTA; } \\
\text { Dentsply-Sirona) } \\
\text { Theracal LC (Th; Bisco) }\end{array}$} & \multirow[b]{2}{*}{$\begin{array}{l}\text { Indirect contact } \\
\text { (SET materials) }\end{array}$} & Cell viability & WST- 1 assay (at 24 h) & $\begin{array}{c}100 \% \text { concentration: Th }>\text { MTA; At } 50 \%, 25 \% \text { and } 10 \% \\
\text { dilutions: Th } \approx \text { MTA } \\
\text { At } 100 \% \text { MTA: cell viability }<70 \% \text { and significantly lower } \\
\text { than Th. }\end{array}$ \\
\hline & & & Cell differentiation & $\begin{array}{l}\text { RT-PCR (DSPP, DMP- } 1 \text { at } 1 \& 3 \mathrm{~d} \text { ) } \\
\text { Q-PCR(DSPP, DMP- } 1 \text { at } 2,5 \& \text { d }) \\
\text { ALP staining (at day } 7 \text { ) } \\
\text { ARS (at day } 14 \text { ) }\end{array}$ & $\begin{array}{c}\text { DSPP } 1 \text { d: MTA }>\text { Th } \approx \text { control; } 3 \mathrm{~d}: \text { MTA } \approx \text { Th }>\text { control } \\
\text { DMP-1 at } 1 \text { and } 3 \mathrm{~d}: \text { MTA } \approx \text { Th } \approx \text { control } \\
\text { DSPP \& DMP-1: upregulated for both materials, } \\
\text { especially at day } 7 . \\
\text { MTA }>\text { Th } \approx \text { control } \\
\text { MTA }>\text { Th }>\text { control }\end{array}$ \\
\hline \multirow[t]{2}{*}{$\begin{array}{l}\text { Mestieri LB et al. } \\
\quad \text { (2017) [71] }\end{array}$} & \multirow[t]{2}{*}{$\begin{array}{l}\text { White MTA Angelus } \\
\text { (MTA-A; Angelus) } \\
\text { White Portland Cement } \\
\text { (PC; Votoran) }\end{array}$} & \multirow[t]{2}{*}{$\begin{array}{l}\text { Indirect contact } \\
\text { (SET materials) }\end{array}$} & Cell viability & Trypan blue assay & $\begin{array}{c}\text { 1:2, 1:3, } 1: 4 \text { and } 1: 8 \text { dilutions: control }>\text { W-MTA-A }>\text { W-PC } \\
1: 6 \text { dilution: } \text { MTA-A } \approx \text { control }>\text { PC } \\
1: 2 \text { dilution: control }>\text { MTA-A }>\text { PC } \\
1: 3 \text { dilution: control }>\text { PC }>\text { MTA-A } \\
\text { 1:4 and 1:6 dilutions: control }>\text { PC }>\text { MTA-A } \\
\text { 1:8 dilution: control }>\text { PC } \approx \text { MTA-A }\end{array}$ \\
\hline & & & Cell Differentiation & ALP activity (at $1,3 \& 7 \mathrm{~d}$ ) & 1,3 and 7 d: MTA-A $\approx \mathrm{PC} \approx$ control \\
\hline \multirow[b]{2}{*}{$\begin{array}{l}\text { Rodrigues EM et al. } \\
\text { (2017) [72] }\end{array}$} & \multirow[b]{2}{*}{$\begin{array}{l}\text { MTA-Plus (MTA-P; } \\
\text { Prevest Denpro) } \\
\text { White MTA Angelus } \\
\text { (MTA-A; Angelus) }\end{array}$} & \multirow[b]{2}{*}{$\begin{array}{c}\text { Indirect contact } \\
\text { (SET } \\
\text { materials)(ISO-10993) }\end{array}$} & Cell viability & $\begin{array}{l}\text { MTT Assay } \\
\text { Flow cytometry- } \\
\text { Annexin V-PI }\end{array}$ & $\begin{array}{c}1: 2 \text { concentration: MTA-P }>\text { MTA-A } \approx \text { control } \\
1: 4 \text { and } 1: 8 \text { concentrations: MTA-P } \approx \text { MTA-A }>\text { control } \\
\text { MTA-A }>\text { live cells than MTA-P } \approx \text { control } \\
\text { MTA-A }>\text { necrotic cells than MTA-P }>\text { control }\end{array}$ \\
\hline & & & Cell differentiation & $\begin{array}{c}\text { ALP activity (at } 1,3 \& 7 \text { days) } \\
\text { ARS (14d) }\end{array}$ & $\begin{array}{c}\text { MTA-A < control < MTA-P after } 7 \text { days. } \\
\text { MTA-A }>\text { MTA-P }>\text { control } \\
\text { Day 1_BMP2 \& OC: MTA-A }>\text { MTA-P > control; ALP: } \\
\text { MTA-A } \approx \text { MTA-P }<\text { control } \\
\text { Day 3_ BMP2: MTA-A > MTA-P > control; OC \& ALP: } \\
\text { MTA-A } \approx \text { MTA-P }<\text { control }\end{array}$ \\
\hline
\end{tabular}


Table 2. Cont

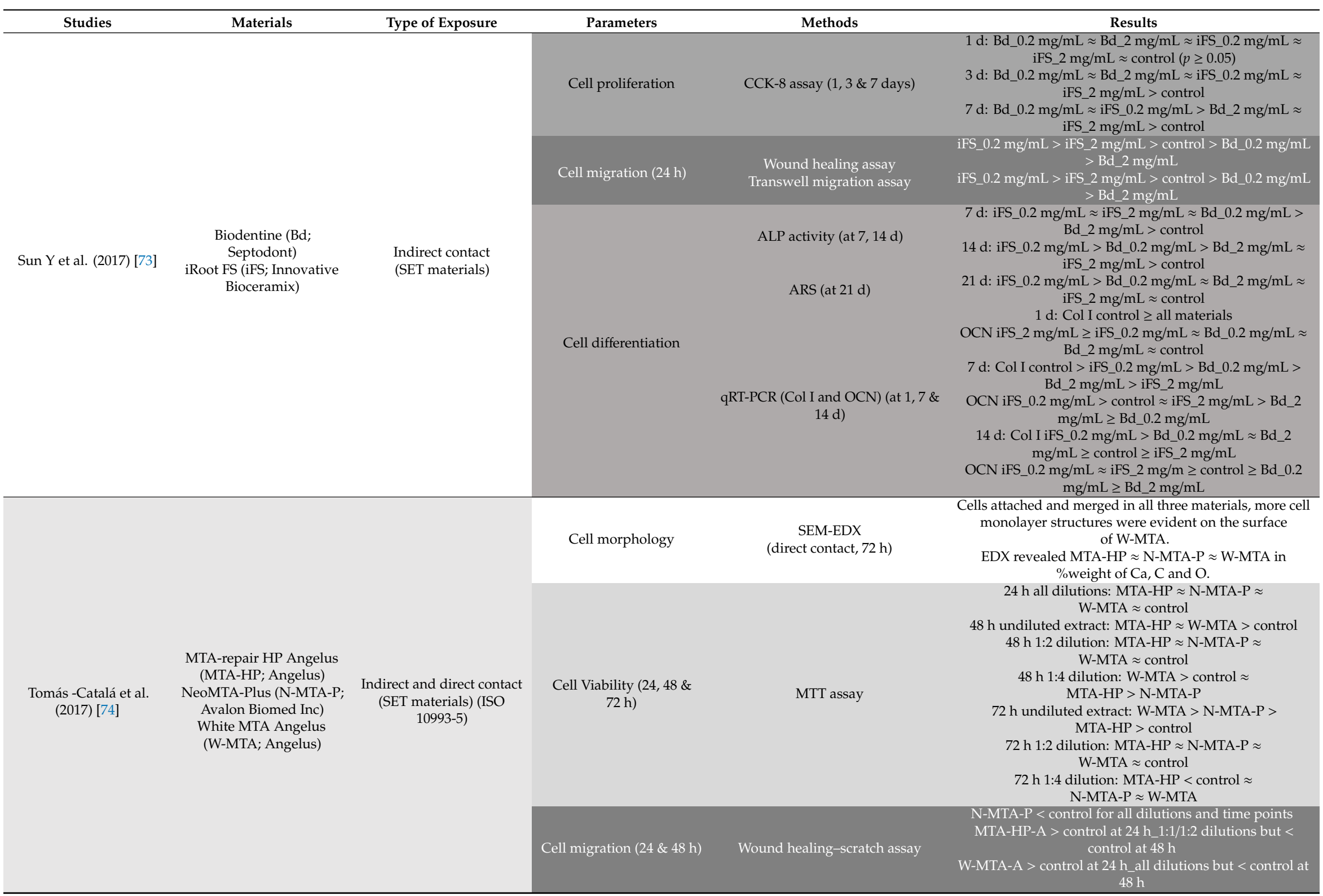


Table 2. Cont

\begin{tabular}{|c|c|c|c|c|c|}
\hline Studies & Materials & Type of Exposure & Parameters & Methods & Results \\
\hline \multirow{5}{*}{$\begin{array}{l}\text { Collado-González M } \\
\text { et al. (2018) [75] }\end{array}$} & \multirow{5}{*}{$\begin{array}{l}\text { GI Equia Forte (EF; GC) } \\
\text { GI Ionostar Molar } \\
\text { (IoM;Voco) }\end{array}$} & \multirow{5}{*}{$\begin{array}{l}\text { Indirect and direct contact } \\
\text { (SET materials) } \\
\text { (ISO 10993-5) }\end{array}$} & $\begin{array}{l}\text { Cell morphology } \\
\text { (indirect contact, } 24 \text { h) }\end{array}$ & $\begin{array}{l}\text { Confocal microscopy } \\
\text { (cytoskeletal F-actin) }\end{array}$ & $\begin{array}{c}1: 1 \text { extracts } E F \approx \begin{array}{c}\text { control (an organized and stretched } \\
\text { stress fiber) }\end{array} \\
\begin{array}{c}1: 1 \text { extracts IoM }<\begin{array}{c}\text { control (cell numbers and stretched } \\
\text { stress fiber) }\end{array}\end{array}\end{array}$ \\
\hline & & & $\begin{array}{l}\text { Cell morphology } \\
\text { (direct contact, } 72 \mathrm{~h})\end{array}$ & SEM & $\mathrm{EF}>\mathrm{IoM}$ (cell attachment, morphology and growth) \\
\hline & & & $\begin{array}{l}\text { Cell Viability (at } 24,48 \& \\
72 \text { h) }\end{array}$ & MTT assay & $\begin{array}{l}24 \text { h all concentrations: Control }>\mathrm{EF} \approx \mathrm{IoM} \\
48 \text { h } 1: 1 \text { dilution: Control } \approx \mathrm{IoM}>\mathrm{EF} \\
48 \text { h } 1: 2 \text { dilution: } \mathrm{IoM} \approx \mathrm{EF} \approx \text { control } \\
48 \text { h } 1: 4 \text { dilution: } \mathrm{IoM} \approx \mathrm{EF} \approx \text { control } \\
72 \text { h } 1: 1 \text { dilution: } \mathrm{EF} \approx \text { control }>\mathrm{IoM} \\
72 \text { h } 1: 2 \text { dilution: control }>\mathrm{IoM} \approx \mathrm{EF} \\
72 \text { h } 1: 4 \text { dilution: control }>\mathrm{EF}>\mathrm{IoM}\end{array}$ \\
\hline & & & $\begin{array}{l}\text { Cell migration (24 and } \\
48 \mathrm{~h})\end{array}$ & Scratch assay & $\begin{array}{c}\text { Control > EF > IoM for all concentrations and } \\
\text { study periods }\end{array}$ \\
\hline & & & Cell differentiation & $\begin{array}{c}\text { Flow cytometry- } \\
\text { Annexin V/7-AAD staining }\end{array}$ & $\begin{array}{l}\text { IoM and } \mathrm{EF} \approx \text { control (the percentage of positive } \\
\text { expression of mesenchymal markers) }\end{array}$ \\
\hline \multirow{4}{*}{$\begin{array}{l}\text { Pedano MS et al. } \\
\text { (2018) [76] }\end{array}$} & \multirow{4}{*}{$\begin{array}{l}\text { Exp-PPL (PPL) } \\
\text { Biodentine (Bd; } \\
\text { Septodont) } \\
\text { Nex-Cem MTA } \\
\text { (Nex-MTA; GC) } \\
\text { Zinc-oxide eugenol } \\
\text { Alganol (ZnO; Kemdent) }\end{array}$} & \multirow{4}{*}{$\begin{array}{l}\text { Indirect contact } \\
\text { (FRESH materials) }\end{array}$} & Cell viability (24 h) & XTT assay & $\begin{array}{l}10 \% \text { eluates: } \mathrm{Bd}>\mathrm{PPL} \approx \mathrm{Nex}-\mathrm{MTA}>\mathrm{ZnO} \\
25 \% \text { eluates: } \mathrm{PPL}>\mathrm{Nex}-\mathrm{MTA}>\mathrm{Bd}>\mathrm{ZnO} \\
50 \% \text { eluates: } \mathrm{PPL} \approx \mathrm{Nex}-\mathrm{MTA}>\mathrm{Bd}>\mathrm{ZnO} \\
100 \% \text { eluates: Nex-MTA }>\text { PPL }>\mathrm{Bd}>\mathrm{ZnO}\end{array}$ \\
\hline & & & $\begin{array}{l}\text { Cell proliferation }(1,4 \& \\
7 \mathrm{~d})\end{array}$ & XTT assay & $\begin{array}{l}10 \% \text { eluates 7d: } \mathrm{PPL} \approx \mathrm{Bd} \approx \text { control }>\text { Nex-MTA }>\mathrm{ZnO} \\
25 \% \text { eluates } 7 \mathrm{~d} \text { : control }>\mathrm{Bd}>\mathrm{PPL}>\mathrm{Nex}-\mathrm{MTA}>\mathrm{ZnO} \\
50 \% \text { eluates } 7 \mathrm{~d} \text { : control }>\mathrm{Bd}>\mathrm{PPL}>\mathrm{Nex}-\mathrm{MTA}>\mathrm{ZnO} \\
100 \% \text { eluates } 7 \mathrm{~d} \text { : control }>\mathrm{PPL} \approx \mathrm{Bd} \approx \text { Nex-MTA }>\mathrm{ZnO}\end{array}$ \\
\hline & & & Cell migration (24 h) & Scratch-wound healing assay & $\begin{array}{l}10 \% \text { and } 25 \% \text { eluates: control } \approx \text { PPL } \approx \text { Nex-MTA }>\text { Bd } \\
50 \% \text { eluates: control } \approx \text { Nex-MTA } \approx \text { PPL }>\text { Bd } \\
100 \% \text { eluates: control }>\text { PPL }>\text { Nex-MTA }>\text { Bd } \\
\text { ALP } 4 \text { d: differentiation medium }>\text { PPL } \approx \text { Bd } \approx \text { Nex-MTA } \\
10 \text { d: differentiation medium } \approx \text { PPL } \approx \text { Bd } \approx \text { Nex-MTA } \\
14 \text { d: differentiation medium }>\text { PPL }>\text { Bd } \approx \text { Nex-MTA }\end{array}$ \\
\hline & & & $\begin{array}{l}\text { Cell differentiation }(4,10 \\
\quad \& 14 \mathrm{~d})\end{array}$ & RT-PCR (ALP, OCN, DSPP) & $\begin{array}{c}\text { OCN } 14 \mathrm{~d}: \text { PPL } \approx \mathrm{Bd}>\text { Nex-MTA } \approx \\
\text { differentiation medium } \\
\text { DSPP } 10 \mathrm{~d}: \text { PPL } \approx \mathrm{Bd} \approx \text { Nex-MTA } \approx \\
\text { differentiation medium } \\
14 \mathrm{~d}: \mathrm{Bd}>\mathrm{PPL}>\text { differentiation medium }>\text { Nex-MTA }\end{array}$ \\
\hline
\end{tabular}


Table 2. Cont

\begin{tabular}{|c|c|c|c|c|c|}
\hline Studies & Materials & Type of Exposure & Parameters & Methods & Results \\
\hline \multirow{3}{*}{$\begin{array}{l}\text { Tomás-Catalá CJ } \\
\text { et al. (2018) [77] }\end{array}$} & \multirow{3}{*}{$\begin{array}{l}\text { Biodentine (Bd; } \\
\text { Septodont)MTA Repair } \\
\text { HP Angelus } \\
\text { (MTA-HP-A; Angelus) } \\
\text { NeoMTA Plus (N-MTA-P; } \\
\text { Avalon Biomed Inc) }\end{array}$} & \multirow{3}{*}{$\begin{array}{l}\text { Indirect and direct contact } \\
\text { (SET materials) (ISO } \\
\text { 10993-5) }\end{array}$} & Cell attachment & SEM-EDX (direct contact, 72 h) & $\begin{array}{l}\text { SEM showed Bd revealed more cells and with better } \\
\text { morphology than MTA-HP-A and N-MTA-P. The EDX } \\
\text { revealed that Bd, MTA-HP-A and N-MTA-P had similar } \\
\text { percentages of Ca, C and O. }\end{array}$ \\
\hline & & & Cell viability & MTT assay $(24,48 \& 72 \mathrm{~h})$ & $\begin{array}{c}\text { Undiluted extract: } \mathrm{Bd}>\mathrm{MTA}-\mathrm{HP}-\mathrm{A}>\mathrm{N}-\mathrm{MTA}-\mathrm{P}> \\
\text { control at } 48 \mathrm{~h} \text { and } 72 \mathrm{~h} \\
\text { 1:2 dilution: } \mathrm{Bd}>\mathrm{MTA}-\mathrm{HP}-\mathrm{A} \approx \mathrm{N}-\mathrm{MTA}-\mathrm{P} \approx \text { control } \\
(p<0.01) \text { at } 48 \mathrm{~h} \text { and } 72 \mathrm{~h} \\
\text { 1:4 dilution: Bd }>\text { N-MTA-P } \approx \text { control }>\text { MTA-HP-A at } \\
72 \mathrm{~h}\end{array}$ \\
\hline & & & Cell migration & Scratch assay (at $24 \& 48$ h) & 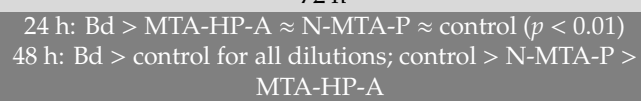 \\
\hline \multirow[t]{2}{*}{ Lee S-M et al. (2019) [78] } & \multirow[t]{2}{*}{$\begin{array}{l}\text { Smart Dentin } \\
\text { Replacement (SDR; } \\
\text { Dentsply-Sirona) } \\
\text { Venus Bulk-fill (VBF; } \\
\text { Hereaus Kulzer) } \\
\text { Beautifil Bulk flowable } \\
\text { (BBF; Shofu) } \\
\text { Filtek Z350 XT Flowable } \\
\text { (ZFF; 3M) }\end{array}$} & \multirow[t]{2}{*}{$\begin{array}{l}\text { Indirect contact } \\
\text { (Set materials) } \\
\text { (ISO 10993-5) }\end{array}$} & Cell viability & $\begin{array}{c}\text { WST assay }(24 \mathrm{~h}) \\
\text { Live/dead Assay } \\
\text { (direct visualization with confocal } \\
\text { microscopy) }\end{array}$ & $\begin{array}{l}\text { 2-mm-cured composite: } \approx 100 \% \text { cell-viability except for } \\
\text { BFF }(49 \%) \\
\text { 4-mm-cured composite: SDR not cytotoxic at all dilutions. } \\
\text { VBF \& BBF statistically different values ( } 71.05 \% \text { and } \\
64.43 \% \text {, respectively) of cell viability at } 100 \% \\
\text { concentration compared to control }(p<0.05) \text { but no } \\
\text { statistically different cell viability compared to control at } \\
25 \% \text { and } 12.5 \% \text { concentrations, respectively }(\sim 100 \% \text {, } \\
\qquad>0.05) \\
6 \text {-mm-cured composite: SDR and BBF were } \sim 69 \% \text { and } \\
\sim 6 \% \text { at } 100 \% \text { concentration }(p<0.05) \text {, and these resins did } \\
\text { not show statistically different cell viability compared to } \\
\text { control at } 25 \% \text { and } 12.5 \%(\sim 100 \%, p>0.05) \text {, respectively. } \\
\text { In contrast, VBF and ZFF did not reach non-cytotoxic } \\
\text { levels }(\sim 100 \%) \text { even at } 12.5 \% \text { dilution. } \\
\text { At } 100 \% \text { concentrations of SDR, VBF, and ZFF, } 6 \text {-mm } \\
\text { cured composite showed } 5 \sim 60 \% \text { live cell numbers } \\
\text { compared to the 2-mm cured group. Another bulk-fill } \\
\text { resin, BBF, had } 5 \sim 35 \% \text { live cells with some dead cells in all } \\
\text { groups. At } 12.5 \% \text {, there were full of live cells at all groups } \\
\text { while the } 4 \text {-mm cured ZFF and the } 6 \text {-mm cured VBF and } \\
\text { ZFF revealed fewer live cells }(\sim 75 \%) \text { than the control. }\end{array}$ \\
\hline & & & $\begin{array}{l}\text { Cell differentiation } \\
\text { ( } 7 \text { days) }\end{array}$ & ALP staining & $\begin{array}{l}\text { 6-mm-cured bulk-fill resins showed significantly lower } \\
\text { ALP staining than the differentiation media control } \\
(p<0.05) \text {, while all 2-mm and 4-mm cured bulk-fill resins } \\
\text { showed similar ALP staining, except for 4-mm-cured BBF. } \\
\text { ALP staining from the bulk-fill resins was ranked as } \\
\text { follows: } 2 \text {-mm }>4 \text {-mm }>6 \text {-mm cured. The flowable resin, } \\
\text { ZFF, exhibited the least amount of ALP staining between } \\
\text { the experimental groups. }\end{array}$ \\
\hline
\end{tabular}


Table 2. Cont

\begin{tabular}{|c|c|c|c|c|c|}
\hline Studies & Materials & Type of Exposure & Parameters & Methods & Results \\
\hline \multirow{4}{*}{$\begin{array}{l}\text { López-García et al. } \\
\text { (2019) [79] }\end{array}$} & \multirow{4}{*}{$\begin{array}{l}\text { Activa Kids (Activa; } \\
\quad \text { Pulpdent) } \\
\text { GI Ionolux (Voco) } \\
\text { Riva Light Cure } \\
\text { (Riva; SDI) }\end{array}$} & \multirow{4}{*}{$\begin{array}{l}\text { Indirect and direct contact } \\
\text { (Set materials) } \\
\text { (ISO 10993-5) }\end{array}$} & $\begin{array}{l}\text { Cell morphology } \\
\text { (indirect contact) }\end{array}$ & Immunofluorescence & Activa $>$ cell density and spreading than Riva $>$ Inolux \\
\hline & & & $\begin{array}{l}\text { Cell attachment/adhesion } \\
\text { (direct contact) }\end{array}$ & SEM & $\begin{array}{l}\text { Activa showed well-adhered fibroblastic cells with } \\
\text { multiple cytoplasmic extensions. } \\
\text { Riva showed less density and fewer cells than Activa. } \\
\text { Ionolux induced drastic reduction in cell density } \\
\text { and attachement. }\end{array}$ \\
\hline & & & Cell viability & MTT assay (1, $2 \& 4$ days) & $\begin{array}{c}24 \mathrm{~h} \text { - Undiluted extracts: Activa } \approx \text { control }>\text { Riva }> \\
\text { Ionolux }(p<0.01) \\
24 \text { h-1:2 dilution: Activa } \approx \text { control } \approx \text { Riva }>\text { Ionolux } \\
24 \text { h-1:4 dilution: Activa } \approx \text { control }>\text { Riva }>\text { Ionolux } \\
48 \text { h-Undiluted extracts: Ionolux }<\text { Activa \& Riva } \\
(p<0.01)<\text { control }(p<0.01) \\
48 \text { h-1:2 dilution: Activa \& Riva \& Ionolux } \approx \text { control } \\
48 \text { h-1:4 dilution: Activa \& Riva \& Ionolux } \approx \text { control } \\
72 \text { h-Undiluted extracts: Control }>\text { Activa }> \\
\text { Riva }>\text { Ionolux } \\
72 \text { h-1:2 dilution: Control }>\text { Activa }>\text { Riva }>\text { Ionolux } \\
72 \text { h-1:4 dilution: Activa } \approx \text { control; Riva \& } \\
\text { Ionolux }<\text { control }\end{array}$ \\
\hline & & & Cell migration & Wound healing assay & $\begin{array}{c}\text { Activa } \approx \text { control at all dilutions except } 1: 2 \text { at } 72 \mathrm{~h} \\
\text { Riva }<\text { migration than control except 1:4 dilution } \\
\text { Ionolux }<\text { migration than control except 1:4 dilution at } \\
24 \mathrm{~h} \text { and } 48 \mathrm{~h}\end{array}$ \\
\hline \multirow{3}{*}{ Dou L et al. (2020) [80] } & \multirow{3}{*}{$\begin{array}{l}\text { Dycal (Dentsply-Sirona) } \\
\text { Pro-Root MTA (MTA; } \\
\text { Dentsply-Sirona) } \\
\text { iRoot BP (iRoot; } \\
\text { Innovative Bioceramix) } \\
\text { Platelet-rich Fibrin (PRF) } \\
\text { Concentrated Growth } \\
\text { Factors (CGF) }\end{array}$} & \multirow{3}{*}{$\begin{array}{l}\text { Indirect contact } \\
\text { (Set materials) }\end{array}$} & Cell viability & $\begin{array}{l}\text { Trypan Blue Staining } \\
\text { (1, } 3 \text { \& } 7 \text { days) } \\
\text { Flow cytometry- } \\
\text { Annexin V-PI } \\
\text { (1, } 3 \& 7 \text { days }) \\
\text { Cell Cycle( } 1,3 \text { \& } 7 \text { days })\end{array}$ & $\begin{array}{l}\text { Dycal }<\text { cell viability than } \mathrm{MTA} \approx \mathrm{iRoot} \approx \mathrm{PRF} \approx \mathrm{CGF} \approx \\
\quad \text { control at } 1,3 \& 7 \text { days } \\
\text { Dycal }>\text { apoptotic cells than MTA } \approx \mathrm{iRoot} \approx \mathrm{CGF} \approx \\
\quad \text { control at } 1,3 \& 7 \text { days } \\
\text { Days } 1 \text { \& } 3 \text { : no significant differences among the groups } \\
\text { Day 7: CGF showed less cells in } \mathrm{G}_{0} / \mathrm{G}_{1} \text {-phase compared } \\
\text { to MTA \& Dycal }\end{array}$ \\
\hline & & & Cell proliferation & CCK-8 & $\begin{array}{l}\text { Day 1: Dycal < cell proliferation than all groups; MTA } \approx \\
\quad \text { iRoot } \approx \text { PRF } \approx \text { CGF } \approx \text { control. } \\
\text { Day 3: PRF \& CGF }>\text { cell proliferation than control \& } \\
\text { MTA, but } \approx \text { iRoot; Dycal < all groups } \\
\text { Day 7: CGF > cell proliferation than iRoot \& MTA, but } \approx \\
\quad \text { control \&PRF; Dycal < all groups }\end{array}$ \\
\hline & & & $\begin{array}{l}\text { Cell differentiation } \\
\text { (1,3 \& } 7 \text { days) }\end{array}$ & ALP staining & $\begin{array}{l}\text { Days } 1 \& 3 \text { : MTA }>\text { ALP-activity than control; Control } \approx \\
\text { iRoot } \approx \text { PRF } \approx \text { CGF } \approx \text { Dycal } \\
\text { Day 7: Dycal }<\text { ALP-activity than CGF; CGF } \approx \text { control } \approx \\
\text { MTA } \approx \text { iRoot } \approx \text { PRF }\end{array}$ \\
\hline
\end{tabular}

* Direct contact was considered when the cells were seeded on top of the materials. When the material was placed on a transwell insert or materials' eluates were used, it was considered

INDIRECT contact. 
Table 3. Included in vivo studies (immature permanent teeth).

\begin{tabular}{|c|c|c|c|c|c|c|c|c|}
\hline Authors & Study Type & Hemostasia & Materials Used & Etched Pulp? & $\begin{array}{l}\text { Evaluation } \\
\text { Period(s) }\end{array}$ & Bridge Formation & Inflammation & Sample \\
\hline $\begin{array}{l}\text { Hebling J et al. } \\
\text { (1999) [81] }\end{array}$ & $\mathrm{CCT}$ & $\begin{array}{l}\text { Sterile cotton } \\
\text { pellets + sterile } \\
\text { saline }\end{array}$ & $\begin{array}{l}\text { Calcium-hydroxide } \\
\text { saline paste (CH) + } \\
\text { calcium-hydroxide } \\
\text { cement (Dycal; } \\
\text { Dentsply-Sirona) } \\
\text { All Bond 2 } \\
\text { (AB2; Bisco) }\end{array}$ & $\begin{array}{l}\text { No (CH), } \\
\text { Yes (AB2) }\end{array}$ & $\begin{array}{l}7 \text { days } \\
30 \text { days } \\
60 \text { days }\end{array}$ & $\begin{array}{l}\text { CH: } 3 / 4 \text { teeth showed complete } \\
\text { bridge formation at } 30 \text { days. } \\
4 / 4 \text { teeth showed complete bridge } \\
\text { below exposed area at } 60 \text { days. } \\
\text { AB2: } 0 / 6 \text { teeth showed completed } \\
\text { bridge at } 30 \text { or } 60 \text { days }(0 / 12 \text { in } \\
\text { total). All of them showed modest } \\
\text { bridge formation at } 60 \text { days. }\end{array}$ & $\begin{array}{l}\text { CH: } 1 / 4 \text { teeth showed moderate and } \\
3 / 4 \text { slight inflammation at day } 7 \text {. } \\
\text { At day } 30,4 / 4 \text { teeth showed slight } \\
\text { inflammatory reaction. No tooth } \\
\text { showed severe inflammation } \\
\text { AB2: } 1 / 6 \text { teeth showed severe } \\
\text { inflammatory reaction, } 3 / 6 \text { moderate } \\
\text { and } 2 / 6 \text { slight inflammation at day } 7 \text {. } \\
\text { At day } 30,3 / 6 \text { showed moderate and } \\
\text { 3/6 slight inflammatory reaction. }\end{array}$ & $\begin{array}{l}32 \text { premolars } \\
\text { (12-15 years } \\
\text { old patients) }\end{array}$ \\
\hline $\begin{array}{c}\text { Do Nascimento } \\
\text { AB et al. } \\
\text { (2000) [82] }\end{array}$ & $\mathrm{CCT}$ & $\begin{array}{l}\text { Sterile paper } \\
\text { cones + sterile } \\
\text { saline }\end{array}$ & $\begin{array}{l}\text { Calcium-hydroxide } \\
\text { saline paste (CH; } \\
\text { Pathfinder associates) } \\
\text { + calcium hydroxide } \\
\text { cement (Dycal; } \\
\text { Dentsply-Sirona)Resin- } \\
\text { modified } \\
\text { glass-ionomer cement } \\
\text { (Vit; Vitrebond; 3M } \\
\text { Oral Care) }\end{array}$ & No & $\begin{array}{c}5 \text { days } \\
30 \text { days } \\
120+\text { days }\end{array}$ & $\begin{array}{l}\text { CH: } 1 / 4 \text { teeth showed complete } \\
\text { bridge at } 30 \text { days. } 5 / 5 \text { teeth showed } \\
\text { complete bridge at } 120+\text { days. } \\
\text { Vit: } 0 / 6 \text { teeth showed complete } \\
\text { bridge at } 30 \text { days. } 0 / 5 \text { teeth showed } \\
\text { complete bridge at } 120+\text { days. }\end{array}$ & $\begin{array}{l}\text { CH: at day } 5,0 / 6 \text { teeth showed no } \\
\text { inflammation, } 4 / 6 \text { teeth showed } \\
\text { slight, } 2 / 6 \text { moderate and } 0 / 6 \text { severe } \\
\text { inflammation. At day } 30, \\
3 / 4 \text { showed slight and } \\
\text { 1/4 moderate inflammation. } \\
\text { Vit: } 0 / 5 \text { teeth showed none } \\
\text { inflammatory reaction, } 1 / 5 \text { teeth } \\
\text { showed slight and } 4 / 5 \text { moderate } \\
\text { inflammation at day } 5 \text {. At day } 30, \\
\text { 2/6 showed slight and } 4 / 6 \text { moderate } \\
\text { inflammatory reaction. }\end{array}$ & $\begin{array}{l}34 \text { premolars } \\
\text { (11-17 years } \\
\text { old patients) }\end{array}$ \\
\hline $\begin{array}{c}\text { Costa CAS et al. } \\
\text { (2001) [83] }\end{array}$ & $\mathrm{CCT}$ & $\begin{array}{l}\text { Sterile paper } \\
\text { cones + sterile } \\
\text { saline }\end{array}$ & $\begin{array}{l}\text { Calcium-hydroxide } \\
\text { saline paste (CH; } \\
\text { Pathfinder associates) } \\
\text { + calcium hydroxide } \\
\text { cement (Dycal; } \\
\text { Dentsply-Sirona) } \\
\text { Clearfil Liner } \\
\text { Bond 2 (CLB2; } \\
\text { Kuraray Noritake) }\end{array}$ & No & $\begin{array}{c}5 \text { days } \\
30 \text { days } \\
120+\text { days }\end{array}$ & $\begin{array}{l}\text { CH: } 1 / 4 \text { teeth showed complete } \\
\text { bridge at } 30 \text { days. } 5 / 5 \text { teeth showed } \\
\text { complete bridge at } 120+\text { days. } \\
\text { CLB2: } 0 / 5 \text { teeth showed complete } \\
\text { bridge at } 30 \text { days. } 0 / 6 \text { teeth showed } \\
\text { complete bridge at } 120+\text { days. }\end{array}$ & $\begin{array}{l}\text { CH: at day } 5,1 / 6 \text { teeth showed none } \\
\text { inflammatory reaction, } 3 / 6 \text { teeth } \\
\text { showed slight, } 2 / 6 \text { moderate and } 0 / 6 \\
\text { severe inflammation. At day } 30 \text {, } \\
\text { 3/4 showed slight and } \\
\text { 1/4 moderate inflammation. } \\
\text { CLB2: } 4 / 6 \text { teeth showed slight and } \\
2 / 6 \text { moderate inflammation at day } 5 \text {. } \\
\text { At day } 30,2 / 5 \text { showed slight and } 3 / 5 \\
\text { moderate inflammatory reaction. }\end{array}$ & $\begin{array}{l}36 \text { premolars } \\
\text { (11-17 years } \\
\text { old patients) }\end{array}$ \\
\hline $\begin{array}{l}\text { Ersin EK et al. } \\
\text { (2005) [84] }\end{array}$ & $\mathrm{CCT}$ & $3 \% \mathrm{H}_{2} \mathrm{O}_{2}$ & $\begin{array}{l}\text { Calcium-hydroxide } \\
\text { saline paste }(\mathrm{CH})+ \\
\text { calcium hydroxide } \\
\text { cement (Dycal; } \\
\text { Dentsply-Sirona) } \\
\text { Prime\&Bond } \\
2.1 \text { (P\&B2.1; } \\
\text { Dentsply-Sirona) }\end{array}$ & No & $\begin{array}{l}7 \text { days } \\
90 \text { days }\end{array}$ & $\begin{array}{l}\text { CH: } 5 / 5 \text { teeth showed complete } \\
\text { bridge after } 90 \text { days. } \\
\text { P\&B2.1: 0/5 teeth showed } \\
\text { complete bridge after } 90 \text { days. }\end{array}$ & $\begin{array}{l}\text { CH: } 5 / 5 \text { teeth showed slight } \\
\text { inflammatory reaction at day } 7 \text {. } \\
\text { P\&B2.1: at } 7 \text { days, } 5 / 5 \text { teeth showed } \\
\text { moderate acute inflammatory } \\
\text { reaction. } 0 / 5 \text { teeth showed severe } \\
\text { inflammation or necrotic tissue. }\end{array}$ & $\begin{array}{l}20 \text { premolars } \\
\text { (mean age } \\
12.6 \text { years) Age } \\
\text { range not } \\
\text { available }\end{array}$ \\
\hline
\end{tabular}


Table 3. Cont.

\begin{tabular}{|c|c|c|c|c|c|c|c|c|}
\hline Authors & Study Type & Hemostasia & Materials Used & Etched Pulp? & $\begin{array}{l}\text { Evaluation } \\
\text { Period(s) }\end{array}$ & Bridge Formation & Inflammation & Sample \\
\hline $\begin{array}{l}\text { Olsson H et al. } \\
\text { (2005) [85] }\end{array}$ & $\mathrm{RCT}$ & $\begin{array}{l}\text { Continuous } \\
\text { irrigation with } \\
\text { sterile saline }\end{array}$ & $\begin{array}{l}\text { Calcium-hydroxide } \\
\text { saline paste }(\mathrm{CH}) \\
\text { Enamel matrix } \\
\text { derivative (EMD; } \\
\text { Emdogain, BIORA) }\end{array}$ & No & 12 weeks & $\begin{array}{l}\text { CH: } 9 / 9 \text { teeth showed complete } \\
\text { bridge at } 12 \text { weeks. } \\
\text { EMD: } 0 / 9 \text { teeth showed complete } \\
\text { bridge at } 12 \text { weeks. }\end{array}$ & 12 weeks & $\begin{array}{l}18 \text { premolars } \\
\text { (12-16 years } \\
\text { old patients) }\end{array}$ \\
\hline $\begin{array}{l}\text { Silva GAB et al. } \\
\text { (2006) [86] }\end{array}$ & $\mathrm{RCT}$ & $\begin{array}{l}\text { Sterile cotton } \\
\text { pellets + sterile } \\
\text { saline }\end{array}$ & $\begin{array}{c}\text { Calcium-hydroxide } \\
\text { powder (CH; PA } \\
\text { Biodinamica) + } \\
\text { calcium hydroxide } \\
\text { cement (Dycal; } \\
\text { Dentsply-Sirona) } \\
\text { Single Bond (SB; 3M } \\
\text { Oral Care) }\end{array}$ & $\begin{array}{l}\text { No (CH), Yes } \\
(10 \%-37 \% ; \mathrm{SB})\end{array}$ & $\begin{array}{l}1 \text { day } \\
3 \text { days } \\
7 \text { days } \\
30 \text { days }\end{array}$ & $\begin{array}{l}\text { CH: } 5 / 5 \text { teeth showed complete } \\
\text { bridge at day } 30 . \\
\text { SB-10\% Etch: 0/5 teeth showed } \\
\text { complete bridge at day } 30 . \\
\text { SB-37\% Etch: } 0 / 5 \text { teeth showed } \\
\text { complete bridge at day } 30 .\end{array}$ & $\begin{array}{l}\text { CH: 0/20 showed severe } \\
\text { inflammatory reaction at days 1-3-7 } \\
\text { or } 30 \text { (5/time period). } 10 / 20 \text { teeth } \\
\text { showed slight and } 10 / 20 \text { teeth } \\
\text { showed moderate inflammation at } \\
1-3-7 \text { or } 30 \text { days. } \\
\text { SB-10\% Etch: } 0 / 20 \text { showed severe } \\
\text { inflammatory reaction at days } 1-3-7 \\
\text { or } 30 \text { (5/time period). } 1 / 20 \text { teeth } \\
\text { showed none/few inflammatory cells } \\
\text { at d1. } 5 / 20 \text { teeth showed slight and } \\
14 / 20 \text { moderate } \\
\text { inflammatory reaction. } \\
\text { SB-37\% Etch: } 0 / 20 \text { showed severe } \\
\text { inflammatory reaction at days } 1-3-7 \\
\text { or } 30 \text { (5/time period). } 1 / 20 \text { teeth } \\
\text { showed none/few inflammatory cells } \\
\text { at d1. 10/20 teeth showed slight and } \\
\text { 9/20 moderate inflammation at 1-3-7 } \\
\text { or 30d. }\end{array}$ & $\begin{array}{l}81 \text { premolars } \\
\text { (12-17 years } \\
\text { old patients) }\end{array}$ \\
\hline $\begin{array}{l}\text { Sawicki L et al. } \\
\text { (2008) [87] }\end{array}$ & RCT & $\begin{array}{l}\text { Sterile cotton } \\
\text { pellets + sterile } \\
\text { saline }\end{array}$ & $\begin{array}{c}\text { Calcium-hydroxide } \\
\text { cement (Life; Kerr) } \\
\text { White Pro-Root MTA } \\
\text { (W-MTA; } \\
\text { Dentsply-Sirona) }\end{array}$ & No & $47+$ days & $\begin{array}{c}\text { Life: } 11 / 14 \text { teeth showed complete } \\
\text { bridge after } 47+\text { days. } \\
\text { WMTA: } 28 / 30 \text { teeth } \\
\text { complete bridge. } \\
4 \text { teeth lost (not reported) }\end{array}$ & $47+$ days! & $\begin{array}{l}48 \text { premolars } \\
\text { (10-18 years } \\
\text { old patients) }\end{array}$ \\
\hline $\begin{array}{l}\text { Azimi S et al. } \\
\text { (2014) [88] }\end{array}$ & RCT & $\begin{array}{l}\text { Sterile cotton } \\
\text { pellets + sterile } \\
\text { saline }\end{array}$ & $\begin{array}{c}\text { White Pro-Root MTA } \\
\text { (W-MTA; } \\
\text { Dentsply-Sirona) } \\
\text { iRoot BP (iRoot; } \\
\text { Innovative } \\
\text { Bioceramix) }\end{array}$ & No & 6 weeks & $\begin{array}{l}\text { WMTA: 8/12 teeth showed } \\
\text { complete bridge formation at } \\
6 \text { weeks. } \\
\text { iRoot: } 7 / 12 \text { teeth showed complete } \\
\text { bridge at } 6 \text { weeks. }\end{array}$ & 6 weeks!! & $\begin{array}{c}24 \text { premolars } \\
(12-16 \\
\text { years old })\end{array}$ \\
\hline
\end{tabular}


Table 3. Cont

\begin{tabular}{|c|c|c|c|c|c|c|c|c|}
\hline Authors & Study Type & Hemostasia & Materials Used & Etched Pulp? & $\begin{array}{c}\text { Evaluation } \\
\text { Period(s) }\end{array}$ & Bridge Formation & Inflammation & Sample \\
\hline $\begin{array}{c}\text { Swarup SJ et al. } \\
\text { (2014) [89] }\end{array}$ & RCT & $\begin{array}{l}\text { Sterile cotton } \\
\text { pellets }+ \text { sterile } \\
\text { saline }\end{array}$ & $\begin{array}{l}\text { Calcium-hydroxide } \\
\text { cement (Dycal; } \\
\text { Dentsply) } \\
\text { MTA Angelus } \\
\text { (MTA-A; Angelus) } \\
\text { Nanohydroxyapatite } \\
\text { (Hap; Orthogran) }\end{array}$ & No & $\begin{array}{l}15 \text { days } \\
30 \text { days }\end{array}$ & $\begin{array}{c}\text { Dycal: } 2 / 5 \text { had a complete bridge } \\
\text { at } 30 \mathrm{~d} \text {. } \\
\text { MTA-A: } 4 / 5 \text { had complete } \\
\text { bridge } 30 \mathrm{~d} \text {. } \\
\text { Hap: } 4 / 5 \text { teeth had complete } \\
\text { bridge. }\end{array}$ & $\begin{array}{c}\text { Dycal: } 4 / 5 \text { showed moderate and } 1 / 5 \\
\text { severe inflammatory reaction day } 15 . \\
1 / 5 \text { teeth showed moderate and } 4 / 5 \\
\text { slight inflammation at day } 30 . \\
\text { MTA-A: } 3 / 5 \text { teeth showed none and } \\
2 / 5 \text { teeth showed slight } \\
\text { inflammatory reaction at day } 15 . \\
\text { At day } 30,4 / 5 \text { teeth showed none or } \\
\text { few inflammations and } 1 / 5 \text { showed } \\
\text { slight inflammatory reaction. } \\
\text { Hap: } 3 / 5 \text { showed moderate } \\
\text { inflammatory reaction and } 2 / 5 \text { severe } \\
\text { inflammation (day } 15) .3 / 5 \text { showed } \\
\text { no inflammation and } 2 / 5 \text { slight } \\
\text { inflammatory reaction at day } 30 \text {. }\end{array}$ & $\begin{array}{c}30 \text { premolar } \\
(11-15 \\
\text { years old })\end{array}$ \\
\hline $\begin{array}{c}\text { Banava S et al. } \\
\text { (2015) [90] }\end{array}$ & RCT & $\begin{array}{l}\text { Sterile cotton } \\
\text { pellets + sterile } \\
\text { saline }\end{array}$ & $\begin{array}{l}\text { Calcium-hydroxide } \\
\text { cement (Dycal; } \\
\text { Dentsply-Sirona) } \\
\text { White-MTA (WMTA; } \\
\text { Dentsply-Sirona)Pulpdent } \\
\text { Multi-Cal Liner } \\
\text { (PML; Pulpdent) }\end{array}$ & t & 6 weeks & $\begin{array}{c}\text { Dycal: } 7 / 8 \text { teeth showed bridge } \\
\text { at } 6 \mathrm{w} . \\
\text { WMTA: } 12 / 16 \text { teeth sowed bridge } \\
\text { formation at } 6 \mathrm{w} \text {. } \\
\text { PML: } 2 / 8 \text { teeth showed bridge } \\
\text { formation at } 6 \mathrm{w} .\end{array}$ & 6 weeks!! & $\begin{array}{c}32 \text { premolar } \\
(13-20 \\
\text { years old })\end{array}$ \\
\hline
\end{tabular}

Table 4. Included in vivo studies (mature permanent teeth).

\begin{tabular}{|c|c|c|c|c|c|c|c|c|}
\hline Authors & Study Type & Hemostasia & Materials Used & Etched Pulp? & $\begin{array}{c}\text { Evaluation } \\
\text { Period(s) }\end{array}$ & Bridge Formation & Inflammation & Sample \\
\hline $\begin{array}{l}\text { Demarco FF } \\
\text { et al. (2001) [91] }\end{array}$ & RCT & $\begin{array}{l}\text { Sterile cotton } \\
\text { pellets + sterile } \\
\text { saline }\end{array}$ & $\begin{array}{c}\text { Calcium-hydroxide } \\
\text { powder (CH; Labrynth } \\
\text { Produtos) + } \\
\text { calcium-hydroxide cement } \\
\text { Hydro C (Dentsply-Sirona) } \\
\text { Scotchbond Multipurpose } \\
\text { (SBMP; 3M) } \\
\text { Clearfil Liner Bond 2 } \\
\text { (CLB2; Kuraray Noritake) }\end{array}$ & $\begin{array}{l}\text { Yes (SBMP), No } \\
\text { (CH and CLB2) }\end{array}$ & $\begin{array}{l}30 \text { days } \\
90 \text { days }\end{array}$ & $\begin{array}{l}\text { CH: } 2 / 2 \text { complete bridges formed } \\
\text { at } 30 \text { and } 2 / 2 \text { at } 90 \text { days } \\
\text { SBMP: } 0 / 4 \text { teeth with bridges } \\
\text { formed at } 30 \text { and } 0 / 4 \text { at } 90 \text { days. } \\
\text { CLB2: } 1 / 4 \text { teeth showed bridge at } \\
30 \text { days. } 2 / 4 \text { teeth showed bridge } \\
\text { formation at } 90 \text { days. }\end{array}$ & $\begin{array}{l}\text { CH: } 0 / 2 \text { teeth showed severe } \\
\text { inflammation or necrosis at } 30 \text { days. } \\
\text { SBMP: } 1 / 4 \text { teeth showed severe } \\
\text { inflammation or necrosis at } 30 \text { days. } \\
\text { CLB2: } 0 / 4 \text { teeth showed severe } \\
\text { inflammation or necrosis at } 30 \text { days. }\end{array}$ & $\begin{array}{l}20 \text { molars } \\
\text { (20-27 years } \\
\text { old patients }\end{array}$ \\
\hline
\end{tabular}


Table 4. Cont

\begin{tabular}{|c|c|c|c|c|c|c|c|c|}
\hline Authors & Study Type & Hemostasia & Materials Used & Etched Pulp? & $\begin{array}{l}\text { Evaluation } \\
\text { Period(s) }\end{array}$ & Bridge Formation & Inflammation & Sample \\
\hline $\begin{array}{l}\text { Accorinte MLR } \\
\text { et al. (2005) [92] }\end{array}$ & RCT & $\begin{array}{l}\text { Sterile cotton } \\
\text { pellets }+ \text { sterile } \\
\text { saline }\end{array}$ & $\begin{array}{c}\text { Calcium-hydroxide } \\
\text { powder (CH; Labrynth } \\
\text { Produtos) + } \\
\text { calcium-hydroxide cement } \\
\text { (Dycal; Dentsply-Sirona) } \\
\text { Scotchbond Multipurpose } \\
\text { (SBMP; 3M Oral Care) }\end{array}$ & $\begin{array}{l}\text { Yes (SBMP), } \\
\text { No (CH) }\end{array}$ & 60 days & $\begin{array}{c}\mathrm{CH}: 100 \%(5 / 5) \text { of the teeth showed } \\
\text { brige formation at day } 60 . \\
\text { SBMP: } 0 \%(0 / 5) \text { of the teeth } \\
\text { showed bridge formation }\end{array}$ & 60 days & $\begin{array}{l}25 \text { premolars } \\
\text { (15-25 years } \\
\text { old patients) }\end{array}$ \\
\hline $\begin{array}{l}\text { Accorinte MLR } \\
\text { et al. (2006) [93] }\end{array}$ & RCT & $\begin{array}{l}\text { Sterile cotton } \\
\text { pellets + sterile } \\
\text { saline }\end{array}$ & $\begin{array}{c}\text { Calcium-hydroxide } \\
\text { powder (CH; Labrynth } \\
\text { Produtos) + } \\
\text { calcium-hydroxide cement } \\
\text { (Dycal; Dentsply-Sirona) } \\
\text { Scotch Bond Multipurpose } \\
\text { (SBMP; 3M Oral Care) }\end{array}$ & $\begin{array}{l}\text { Yes (SBMP), } \\
\text { No (CH) }\end{array}$ & $\begin{array}{l}30 \text { days } \\
60 \text { days }\end{array}$ & $\begin{array}{l}\text { CH: } 100 \%(5 / 5) \text { of the teeth showed } \\
\text { brige formation at } 30 \text { and } 60 \text { days. } \\
\text { SBMP: } 0 \%(0 / 5) \text { of the teeth showed } \\
\text { bridge formation at } 30 \text { or } 60 \text { days. }\end{array}$ & $\begin{array}{l}\text { CH: no to mild inflammatory } \\
\text { reaction all teeth }(5 / 5) \text { at } 30 \text { days. } \\
\text { SBMP: } 2 / 5 \text { teeth }(40 \%) \text { showed pulp } \\
\text { necrosis at day } 30 .\end{array}$ & $\begin{array}{l}40 \text { premolars } \\
\text { (15-25 years } \\
\text { old patients) }\end{array}$ \\
\hline $\begin{array}{l}\text { Iwamoto CE et } \\
\text { al. (2006) [94] }\end{array}$ & RCT & $\begin{array}{l}\text { Sterile cotton } \\
\text { pellets }+ \text { sterile } \\
\text { saline }\end{array}$ & $\begin{array}{l}\text { Calcium-hydroxide cement } \\
\text { (Dycal; Dentsply-Sirona) } \\
\text { White Pro-Root MTA } \\
\text { (W-MTA; Dentsply-Sirona) }\end{array}$ & No & $112+$ days & $\begin{array}{c}\text { Dycal: } 18 / 23 \text { teeth developed } \\
\text { bridge formation at } 110+\text { days. } \\
\text { W-MTA: } 20 / 22 \text { teeth developed } \\
\text { bridge formation. }\end{array}$ & $112+$ days & $\begin{array}{c}48 \text { molars } \\
\text { (18-60 years } \\
\text { old patients) }\end{array}$ \\
\hline $\begin{array}{l}\text { Accorinte MLR } \\
\text { et al. (2007) [95] }\end{array}$ & СCT & $\begin{array}{l}\text { Sterile cotton } \\
\text { pellets + sterile } \\
\text { saline; sterile } \\
\text { cotton pellets + } \\
2.5 \% \text { sodium } \\
\text { hypochlorite }\end{array}$ & $\begin{array}{l}\text { Calcium-hydroxide cement } \\
\text { (Life; Kerr) }\end{array}$ & No & $\begin{array}{l}30 \text { days } \\
60 \text { days }\end{array}$ & $\begin{array}{l}\text { Life: } 2 / 10 \text { teeth }(20 \%) \text { showed } \\
\text { bridge formation at } 30 \text { days. } \\
\text { At day } 60,6 / 10 \text { teeth showed } \\
\text { complete bridge formation. At day } \\
\text { 60, only } 1 / 10 \text { teeth showed } \\
\text { absent bridge. }\end{array}$ & $\begin{array}{l}\text { Life: At day } 30,8 / 10 \text { teeth showed no } \\
\text { inflammation and } 1 / 10 \text { showed mild } \\
\text { inflammatory reaction. } \\
\text { Only } 1 / 10 \text { teeth showed severe } \\
\text { inflammation. }\end{array}$ & $\begin{array}{l}40 \text { premolars } \\
\text { (15-30 years } \\
\text { old patients) }\end{array}$ \\
\hline $\begin{array}{l}\text { Elias RV et al. } \\
\text { (2007) [96] }\end{array}$ & RCT & $\begin{array}{l}\text { Sterile cotton } \\
\text { pellets }+2.5 \% \\
\text { sodium } \\
\text { hypochlorite }\end{array}$ & $\begin{array}{c}\text { Calcium-hydroxide } \\
\text { powder (CH; Labrynth } \\
\text { Produtos) + } \\
\text { calcium-hydroxide cement } \\
\text { Hydro C (Dentsply-Sirona) } \\
\text { Clearfil SE Bond (CSE; } \\
\text { Kuraray Noritake) }\end{array}$ & No & $\begin{array}{l}30 \text { day } \\
90 \text { days }\end{array}$ & $\begin{array}{l}\text { CH: } 5 / 5 \text { complete bridge after } \\
30 \text { days and } 5 / 5 \text { at } 90 \text { days. } \\
\text { CSE: } 1 / 8 \text { specimens showed dentin } \\
\text { deposition at the interface } \\
\text { (complete bridge) at } 90 \text { days. } 0 / 8 \\
\text { teeth showed complete bridge at } \\
30 \text { days. } 3 / 8 \text { specimens showed no } \\
\text { dentin deposition at all. }\end{array}$ & $\begin{array}{c}\text { CH: } 5 / 5 \text { teeth showed none or slight } \\
\text { inflammation after } 30 \text { days for } \\
\text { all specimens. } \\
\text { CSE: } 4 / 8 \text { specimens showed no } \\
\text { inflammation; } 3 / 8 \text { specimens slight } \\
\text { inflamm; } 1 / 8 \text { severe inflammation at } \\
30 \text { days. }\end{array}$ & $\begin{array}{c}26 \text { molars } \\
\text { (average } \\
25 \text { years) } \\
\text { Age range } \\
\text { not provided }\end{array}$ \\
\hline $\begin{array}{c}\text { Accorinte MLR } \\
\text { et al. } \\
\text { (2008) [53] }\end{array}$ & CCT & $\begin{array}{l}\text { Sterile cotton } \\
\text { pellets }+ \text { sterile } \\
\text { saline }\end{array}$ & $\begin{array}{c}\text { Calcium-hydroxide cement } \\
\text { (Life; Kerr) } \\
\text { Pro-Root MTA (MTA; } \\
\text { Dentsply-Sirona) }\end{array}$ & No & $\begin{array}{l}30 \text { days } \\
60 \text { days }\end{array}$ & $\begin{array}{l}\text { Life: } 2 / 10 \text { teeth showed complete } \\
\text { bridge at } 30 \text { days and } 6 / 10 \\
\text { complete bridge after } 60 \text { days } \\
\text { MTA: } 3 / 10 \text { teeth showed complete } \\
\text { bridge at } 30 \text { days and } 5 / 10 \text { teeth } \\
\text { complete bridge after } 60 \text { days }\end{array}$ & $\begin{array}{c}\text { Life: all teeth showed absent }(8 / 10) \\
\text { or mild (2/10) inflammatory reaction } \\
\text { at } 30 \text { days. } 1 / 10 \text { teeth showed slight } \\
\text { and } 1 / 10 \text { moderate } \\
\text { inflammatory reaction. } \\
\text { No teeth showed necrosis or abscess } \\
\text { formation at } 30 \text { days.MTA: all teeth } \\
\text { showed absent }(9 / 10) \text { or mild }(1 / 10) \\
\text { inflammatory reaction at } 30 \text { days. } \\
\text { No teeth showed necrosis or abscess } \\
\text { formation at } 30 \text { days. }\end{array}$ & $\begin{array}{l}40 \text { premolars } \\
\text { (15-30 years } \\
\text { old patients) }\end{array}$ \\
\hline
\end{tabular}


Table 4. Cont

\begin{tabular}{|c|c|c|c|c|c|c|c|c|}
\hline Authors & Study Type & Hemostasia & Materials Used & Etched Pulp? & $\begin{array}{c}\text { Evaluation } \\
\text { Period(s) }\end{array}$ & Bridge Formation & Inflammation & Sample \\
\hline $\begin{array}{l}\text { Accorinte MLR } \\
\text { et al. (2008) [52] }\end{array}$ & CCT & $\begin{array}{l}\text { Sterile cotton } \\
\text { pellets + sterile } \\
\text { saline }\end{array}$ & $\begin{array}{c}\text { Calcium-hydroxide } \\
\text { powder (CH) + } \\
\text { calcium-hydroxide cement } \\
\text { (Life; Kerr) } \\
\text { MTA Angelus (MTA-A; } \\
\text { Angelus) }\end{array}$ & No & $\begin{array}{l}30 \text { days } \\
60 \text { days }\end{array}$ & $\begin{array}{c}\text { CH: } 6 / 10 \text { teeth showed complete } \\
\text { bridge at } 30 \text { days and } 8 / 10 \text { teeth } \\
\text { showed complete bridge after } \\
\quad 60 \text { days } \\
\text { MTA-A: } 4 / 10 \text { teeth showed } \\
\text { complete bridge at } 30 \text { days and } \\
\text { 7/10 complete bridge after } 60 \text { days }\end{array}$ & $\begin{array}{l}\text { CH: } 8 / 10 \text { teeth showed absent or } \\
\text { slight inflammatory reaction at } \\
30 \text { days. } \\
\text { No teeth showed necrosis or abscess } \\
\text { formation at } 30 \text { days. } \\
\text { MTA-A: } 8 / 10 \text { teeth showed absent or } \\
\text { slight inflammatory reaction at } \\
30 \text { days. } 1 / 10 \text { teeth showed severe } \\
\text { inflammation (abscess formation) at } \\
30 \text { days. }\end{array}$ & $\begin{array}{l}40 \text { premolars } \\
\text { (15-30 years } \\
\text { old patients) }\end{array}$ \\
\hline $\begin{array}{l}\text { Accorinte MLR } \\
\text { et al. (2008) [97] }\end{array}$ & $\mathrm{CCT}$ & $\begin{array}{l}\text { Sterile cotton } \\
\text { pellets + sterile } \\
\text { saline }\end{array}$ & $\begin{array}{l}\text { Calcium-hydroxide cement } \\
\text { (Dycal; Dentsply-Sirona) } \\
\text { Clearfil Liner Bond 2V } \\
\text { (C2V; Kuraray Noritake) } \\
\text { Clearfil SE Bond (CSE; } \\
\text { Kuraray Noritake) }\end{array}$ & No & $\begin{array}{l}30 \text { days } \\
90 \text { days }\end{array}$ & $\begin{array}{l}\text { Dycal: } 8 / 9 \text { teeth with complete } \\
\text { bridge formed at day } 90 \text {. No } \mathrm{CH} \\
\text { treated teeth extracted at } 30 \text { days. } \\
\text { C2V: } 1 / 4 \text { teeth complete bridge at } \\
30 \text { days. } 1 / 6 \text { teeth with complete } \\
\text { bridge at day } 90 \text { and } 2 / 6 \text { with } \\
\text { absence or discrete bridge } \\
\text { CSE: } 0 / 5 \text { teeth complete bridge at } \\
30 \text { days. } 1 / 6 \text { teeth with complete } \\
\text { bridge at day } 90 \text { and } 4 / 6 \text { with } \\
\text { absence or discrete bridge }\end{array}$ & $\begin{array}{c}\text { Dycal: no teeth were extracted at } \\
30 \text { days for } \mathrm{CH} \text { group. } \\
\text { C2V: All teeth had slight }(2 / 6) \text { or } \\
\text { moderate }(3 / 6) \text { inflammation after } \\
30 \text { days including } 1 / 6 \text { teeth } \\
\text { with abscess. } \\
\text { CSE: All teeth had slight }(3 / 6) \text { or } \\
\text { moderate }(2 / 6) \text { inflammation after } \\
30 \text { days including } 1 / 6 \text { teeth } \\
\text { with abscess. }\end{array}$ & $\begin{array}{l}34 \text { premolars } \\
\text { (15-30 years } \\
\text { old patients) }\end{array}$ \\
\hline $\begin{array}{l}\text { Lu Y et al. } \\
\text { (2008) [98] }\end{array}$ & RCT & $\begin{array}{l}\text { Sterile cotton } \\
\text { pellets }+2 \% \\
\text { chlorhexidine }+ \\
\text { sterile saline }\end{array}$ & $\begin{array}{l}\text { Calcium-hydroxide cement } \\
\text { (Dycal; Dentsply-Sirona) } \\
\text { Clearfil SE Bond (CSE; } \\
\text { Kuraray Noritake) }\end{array}$ & No & $\begin{array}{l}7 \text { days } \\
30 \text { days } \\
90 \text { days }\end{array}$ & $\begin{array}{l}\text { Dycal: } 1 / 6 \text { teeth complete bridge at } \\
\text { day } 30.5 / 7 \text { teeth complete bridge } \\
\text { deposits after } 90 \text { days. } \\
\text { CSE: } 0 / 7 \text { teeth complete bridge } \\
\text { after } 30 \text { and } 0 / 7 \text { at } 90 \text { days. }\end{array}$ & $\begin{array}{l}\text { Dycal: } 2 / 7 \text { teeth showed slight, } 4 / 7 \\
\text { moderate and } 1 / 7 \text { teeth showed } \\
\text { severe inflammatory reaction at day } \\
\text { 7. At day } 30,5 / 6 \text { teeth showed slight } \\
\text { and } 1 / 6 \text { moderate } \\
\text { inflammatory reaction. } \\
\text { CSE: } 6 / 7 \text { teeth showed slight and } 1 / 7 \\
\text { moderate inflammatory reaction at } \\
\text { both } 7 \text { and } 30 \text { days. }\end{array}$ & $\begin{array}{c}45 \text { molars } \\
\text { (20-25 years } \\
\text { old patients) }\end{array}$ \\
\hline $\begin{array}{l}\text { Min K-S et al. } \\
(2008) \text { [99] }\end{array}$ & RCT & $\begin{array}{c}\text { Sterile cotton } \\
\text { pellets + sterile } \\
\text { saline }\end{array}$ & $\begin{array}{l}\text { Calcium-hydroxide cement } \\
\text { (Dycal; Dentsply-Sirona) } \\
\text { Pro-Root MTA (MTA; } \\
\text { Dentsply-Sirona) }\end{array}$ & No & 2 months & $\begin{array}{l}\text { Dycal: } 6 / 10 \text { teeth complete bridge. } \\
\text { 4/10 total absence of bridge. } \\
\text { MTA: } 100 \% \text { complete } \\
\text { bridge formation. } \\
\text { Bridges were significantly thicker } \\
\text { with MTA }\end{array}$ & 2 months!! & $\begin{array}{c}20 \text { molars } \\
(2-50 \text { years } \\
\text { old patients) }\end{array}$ \\
\hline
\end{tabular}


Table 4. Cont

\begin{tabular}{|c|c|c|c|c|c|c|c|c|}
\hline Authors & Study Type & Hemostasia & Materials Used & Etched Pulp? & $\begin{array}{l}\text { Evaluation } \\
\text { Period(s) }\end{array}$ & Bridge Formation & Inflammation & Sample \\
\hline $\begin{array}{l}\text { Nair PNR et al. } \\
\text { (2008) [100] }\end{array}$ & RCT & $\begin{array}{l}1 \% \text { sodium } \\
\text { hypochlorite }+ \\
\text { sterile saline }+ \\
\text { paper points }\end{array}$ & $\begin{array}{l}\text { Calcium-hydroxide cement } \\
\text { (Dycal; Dentsply-Sirona) } \\
\text { Pro-Root MTA (MTA; } \\
\text { Dentsply-Sirona) }\end{array}$ & No & $\begin{array}{l}7 \text { days } \\
30 \text { days } \\
90 \text { days }\end{array}$ & $\begin{array}{l}\text { Dycal: } 1 / 5 \text { teeth complete bridge } \\
\text { and } 3 / 5 \text { partial bridge at day } 30 \text {. } \\
\text { At day } 90,2 / 4 \text { teeth partial bridge } \\
\text { and } 0 / 4 \text { complete bridge at } 90 \text { days. } \\
\text { Thick bridges with tunnel defects. } \\
\text { MTA: } 3 / 6 \text { teeth with complete } \\
\text { bridge formation at day } 30.4 / 5 \\
\text { teeth complete bridge formation at } \\
\text { day } 90 \text {. } \\
\text { MTA showed thicker and less } \\
\text { variable bridges than Dycal. }\end{array}$ & $\begin{array}{c}\text { Dycal: at day } 7,2 / 4 \text { teeth showed } \\
\text { inflammatory reaction, } 1 \text { of them } \\
\text { with severe inflammation. } \\
\text { At day } 30,1 / 5 \text { teeth showed severe } \\
\text { inflammatory reaction. } \\
\text { MTA: } 5 / 6 \text { teeth showed absence of } \\
\text { inflammatory reaction and } 1 / 6 \\
\text { showed slight inflammation at day } 7 \text {. } \\
\text { At day } 30,6 / 6 \text { samples showed no } \\
\text { inflammatory reaction. }\end{array}$ & $\begin{array}{l}33 \text { molars } \\
\text { (18-30 years } \\
\text { old patients) }\end{array}$ \\
\hline $\begin{array}{l}\text { Accorinte } \\
\text { MLR et al. } \\
\text { (2009) [101] }\end{array}$ & RCT & $\begin{array}{l}\text { Sterile cotton } \\
\text { pellets + sterile } \\
\text { saline }\end{array}$ & $\begin{array}{l}\text { Pro-Root MTA (MTA; } \\
\text { Dentsply-Sirona) } \\
\text { MTA Angelus } \\
\text { (MTA-A; Angelus) }\end{array}$ & No & $\begin{array}{l}30 \text { days } \\
60 \text { days }\end{array}$ & $\begin{array}{c}\text { MTA: } 5 / 8 \text { teeth had complete } \\
\text { bridge at day } 30 \text {. } \\
\text { 5/9 teeth showed complete bridge } \\
\text { at day } 60 . \text { MTA-A: } 5 / 8 \text { teeth had } \\
\text { complete bridge at day } 30 . \\
\text { 6/10 teeth showed complete bridge } \\
\text { at day } 60 .\end{array}$ & $\begin{array}{l}\text { MTA: } 2 / 8 \text { teeth showed no } \\
\text { inflammation and } 6 / 8 \text { slight } \\
\text { inflammatory reaction at day } 30 \text {. } \\
\text { MTA-A: } 3 / 8 \text { teeth showed no } \\
\text { inflammation at day } 30.4 / 8 \text { showed } \\
\text { mild inflammatory reaction and } 1 \\
\text { tooth showed abscess at day } 30 .\end{array}$ & $\begin{array}{l}35 \text { premolars } \\
\text { (25-42 years } \\
\text { old patients) }\end{array}$ \\
\hline $\begin{array}{c}\text { Kiatwateeratana } \\
\text { T et al. } \\
\text { (2009) [102] }\end{array}$ & RCT & $\begin{array}{l}\text { Moistened } \\
\text { sterile cotton } \\
\text { pellets }\end{array}$ & $\begin{array}{c}\text { Calcium-hydroxide } \\
\text { powder (CH) } \\
\text { Enamel matrix derivative } \\
\text { (EMD; Emdogain, BIORA) } \\
\end{array}$ & No & 6 months & $\begin{array}{c}\text { CH: 10/13 teeth showed complete } \\
\text { bridge formation } \\
\text { EMD: no tooth showed } \\
\text { bridge formation }\end{array}$ & 6 months! & $\begin{array}{c}26 \text { premolars } \\
(13-22 \\
\text { years old })\end{array}$ \\
\hline $\begin{array}{l}\text { Parolia A et al. } \\
\text { (2010) [103] }\end{array}$ & ССТ & $\begin{array}{l}\text { Moistened } \\
\text { sterile cotton } \\
\text { pellets }\end{array}$ & $\begin{array}{l}\text { Calcium-hydroxide cement } \\
\text { (Dycal; Dentsply-Sirona) } \\
\text { Pro-Root MTA (MTA; } \\
\text { Dentsply-Sirona)Propolis } \\
\text { powder (Propolis; } \\
\text { Ecuadorian Rainforest LLC) } \\
\text { mixed with 70\% ethanol } \\
\end{array}$ & No & $\begin{array}{l}15 \text { days } \\
45 \text { days }\end{array}$ & $\begin{array}{l}\text { Dycal: } 5 / 6 \text { teeth showed bridge } \\
\text { formation at } 45 \text { days. } \\
\text { MTA: } 6 / 6 \text { teeth showed bridge } \\
\text { formation at day } 45 \text {. } \\
\text { Propolis: } 6 / 6 \text { teeth showed bridge } \\
\text { formation at } 45 \text { days. }\end{array}$ & $\begin{array}{l}\text { Dycal: } 6 / 6 \text { teeth showed none or } \\
\text { mild inflammation at day } 15 . \\
\text { MTA: all teeth }(6 / 6) \text { showed none or } \\
\text { mild inflammation at day } 15 . \\
\text { Propolis: } 6 / 6 \text { teeth showed none or } \\
\text { mild inflammation at day } 15 .\end{array}$ & $\begin{array}{c}36 \text { premolars } \\
(15-25 \\
\text { years old })\end{array}$ \\
\hline $\begin{array}{l}\text { Nowicka A et al. } \\
\text { (2013) [104] }\end{array}$ & $\mathrm{CCT}$ & $\begin{array}{l}\text { Sterile cotton } \\
\text { pellets + sterile } \\
\text { saline }\end{array}$ & $\begin{array}{c}\text { White Pro-Root MTA } \\
\text { (W-MTA; Dentsply-Sirona) } \\
\text { Biodentine (Biodentine; } \\
\text { Septodont) }\end{array}$ & No & 6 weeks & $\begin{array}{l}\text { W-MTA: } 7 / 11 \text { teeth had complete } \\
\text { bridge. All teeth formed bridges. } \\
\text { Biodentine: } 6 / 11 \text { teeth had } \\
\text { complete bridge. All teeth } \\
\text { formed bridges. }\end{array}$ & 6 weeks! & $\begin{array}{l}28 \text { molars } \\
\text { (19-28 years } \\
\text { old patients) }\end{array}$ \\
\hline $\begin{array}{l}\text { Nowicka A et al. } \\
\text { (2016) [105] }\end{array}$ & RCT & $\begin{array}{l}\text { Sterile cotton } \\
\text { pellets + sterile } \\
\text { saline }\end{array}$ & $\begin{array}{c}\text { Calcium hydroxide paste } \\
\text { (Calcipast; Cerkamed) + } \\
\text { Calcium hydroxide cement } \\
\text { (Life, Kerr) } \\
\text { Single Bond Universal } \\
\text { (SBU; 3M Oral Care) }\end{array}$ & No & 6 weeks & $\begin{array}{l}\text { Calcipast: all teeth showed bridge } \\
\text { formation with } 5 / 11 \text { teeth showing } \\
\text { complete bridge at } 6 \mathrm{wk} \text { SBU: none } \\
\text { of the teeth showed complete } \\
\text { bridge formation. } 7 / 11 \text { teeth } \\
\text { showed no bridge formation at } \\
6 \text { wks. }\end{array}$ & 6 weeks! & $\begin{array}{l}28 \text { molars } \\
\text { (19-28 years } \\
\text { old patients) }\end{array}$ \\
\hline
\end{tabular}


Table 4. Cont

\begin{tabular}{|c|c|c|c|c|c|c|c|c|}
\hline Authors & Study Type & Hemostasia & Materials Used & Etched Pulp? & $\begin{array}{l}\text { Evaluation } \\
\text { Period(s) }\end{array}$ & Bridge Formation & Inflammation & Sample \\
\hline $\begin{array}{l}\text { Bakhtiar H et al. } \\
\text { (2017) [106] }\end{array}$ & RCT & $\begin{array}{l}\text { Sterile cotton } \\
\text { pellets }\end{array}$ & $\begin{array}{l}\text { Theracal LC (Theracal; } \\
\text { Bisco) } \\
\text { Biodentine (Biodentine; } \\
\text { Septodont) } \\
\text { Pro-Root MTA (W-MTA; } \\
\text { Dentsply-Sirona) }\end{array}$ & No & 8 weeks & $\begin{array}{l}\text { Theracal: } 2 / 9 \text { teeth showed no } \\
\text { bridge formation at } 8 \text { wks. } 1 / 9 \\
\text { teeth showed complete bridge at } \\
8 \text { wks. } \\
\text { Biodentine: All teeth }(9 / 9) \text { showed } \\
\text { a complete bridge formation at } \\
8 \text { wks. } \\
\text { W-MTA: } 5 / 9 \text { teeth showed a } \\
\text { complete bridge at } 8 \text { wks. } 4 / 9 \text { teeth } \\
\text { showed an incomplete bridge at } \\
\quad 8 \text { wks. }\end{array}$ & 8 weeks! & $\begin{array}{l}27 \text { molars } \\
\text { (18-32 years } \\
\text { old patients) }\end{array}$ \\
\hline $\begin{array}{l}\text { Jalan AL et al. } \\
\text { (2017) [107] }\end{array}$ & RCT & $\begin{array}{l}\text { Sterile cotton } \\
\text { pellets + sterile } \\
\text { saline }\end{array}$ & $\begin{array}{c}\text { Calcium-hydroxide cement } \\
\text { (Dycal; Dentsply-Sirona) } \\
\text { Biodentine (Biodentine; } \\
\text { Septodont) }\end{array}$ & No & 45 days & $\begin{array}{c}\text { Dycal: } 1 / 20 \text { teeth showed no } \\
\text { bridge formation. } 4 / 20 \text { teeth } \\
\text { showed a complete bridge. } \\
\text { Biodentine: all teeth showed } \\
\text { bridge formation. } 16 / 20 \text { teeth } \\
\text { complete bridge. }\end{array}$ & 45 days & $\begin{array}{c}40 \text { premolars } \\
\text { (15-25 } \\
\text { years old) }\end{array}$ \\
\hline $\begin{array}{l}\text { Mehrvarzfar } \\
\text { P et al. } \\
\text { (2018) [108] }\end{array}$ & RCT & $\begin{array}{l}\text { Sterile cotton } \\
\text { pellets + sterile } \\
\text { saline }\end{array}$ & $\begin{array}{c}\text { White Pro-Root MTA } \\
\text { (W-MTA; Dentsply-Sirona) } \\
\text { W-MTA + Treated Dentin } \\
\text { Matrix (W-MTA/TDM; } \\
\text { Dentsply-Sirona) }\end{array}$ & No & 6 weeks & $\begin{array}{l}\text { W-MTA: } 11 / 11 \text { complete bridge } \\
\text { was present in all specimens at } \\
6 \mathrm{wk} \text {. } \\
\text { W-MTA/TDM: } 11 / 11 \text { complete } \\
\text { bridge was present in all } \\
\text { specimens at } 6 \mathrm{wks} .\end{array}$ & 6 weeks! & $\begin{array}{l}26 \text { molars } \\
\text { (15-31 years } \\
\text { old patients) }\end{array}$ \\
\hline
\end{tabular}




\subsection{Risk of Bias in Individual Studies}

For the risk of bias of in vivo studies and for the meta-analysis, the Cochrane Handbook for Systematic Reviews of Interventions 6 [109] and the Review Manager 5.3 software [110] provided by the Cochrane collaboration (www.cochrane.org) were followed. The risk of bias of each individual study can be found next to each forest plot in the meta-analysis (Figures 2-5).

\subsection{Data Analysis}

\subsubsection{Data Synthesis}

For the in vivo studies, quantitative analysis was performed with Review Manager 5.3 (Revman) software provided by the Cochrane Collaboration (www.cochrane.org). Dichotomous data were presented in forest plots as odds ratios (OR) with 95\% confidence intervals (CIs) (Figures 2-5). When the heterogeneity of the studies was considered "low" $\left(\mathrm{I}^{2}<50 \%\right)$, "fixed effects" were considered. When heterogeneity was "high" or when it was not possible to measure (no direct comparisons available), "random effects" were evaluated. The difference in the effect between different materials was considered statistically significant when $p<0.05$. Instead of the "odds ratio," the "relative risk" (RR) was presented in Tables 5-8 for the sake of simplicity in the interpretation of the results [109].

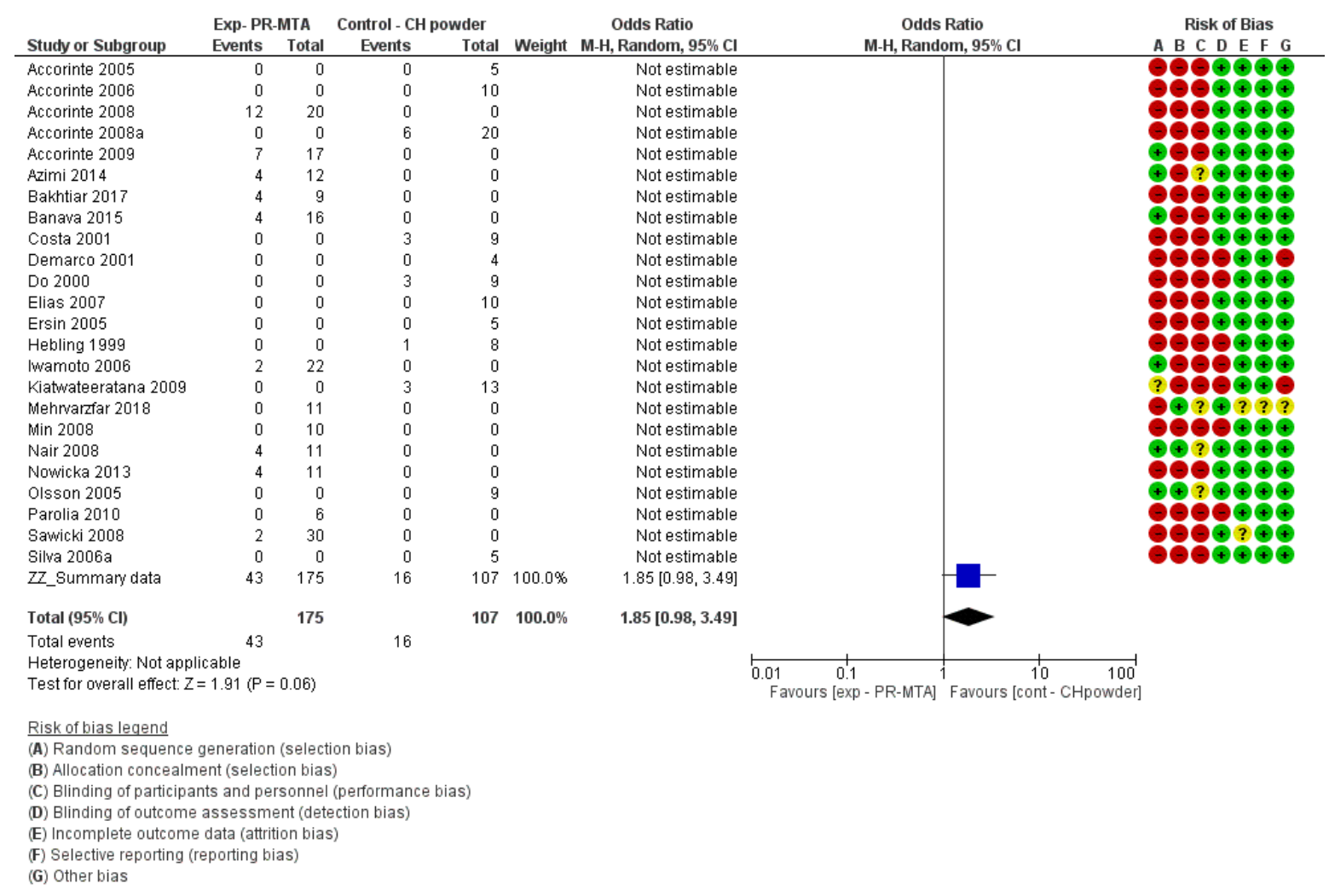

Figure 2. Forest plot and risk of bias of the studies evaluating the formation of a complete reparative bridge comparing calcium-hydroxide powder ( $\mathrm{CH}$ powder) with Pro-Root MTA (Dentsply-Sirona) (odds ratio (95\% CI), random effects). An "event" was considered the lack of a complete hard bridge after 30 days of pulp-capping. 


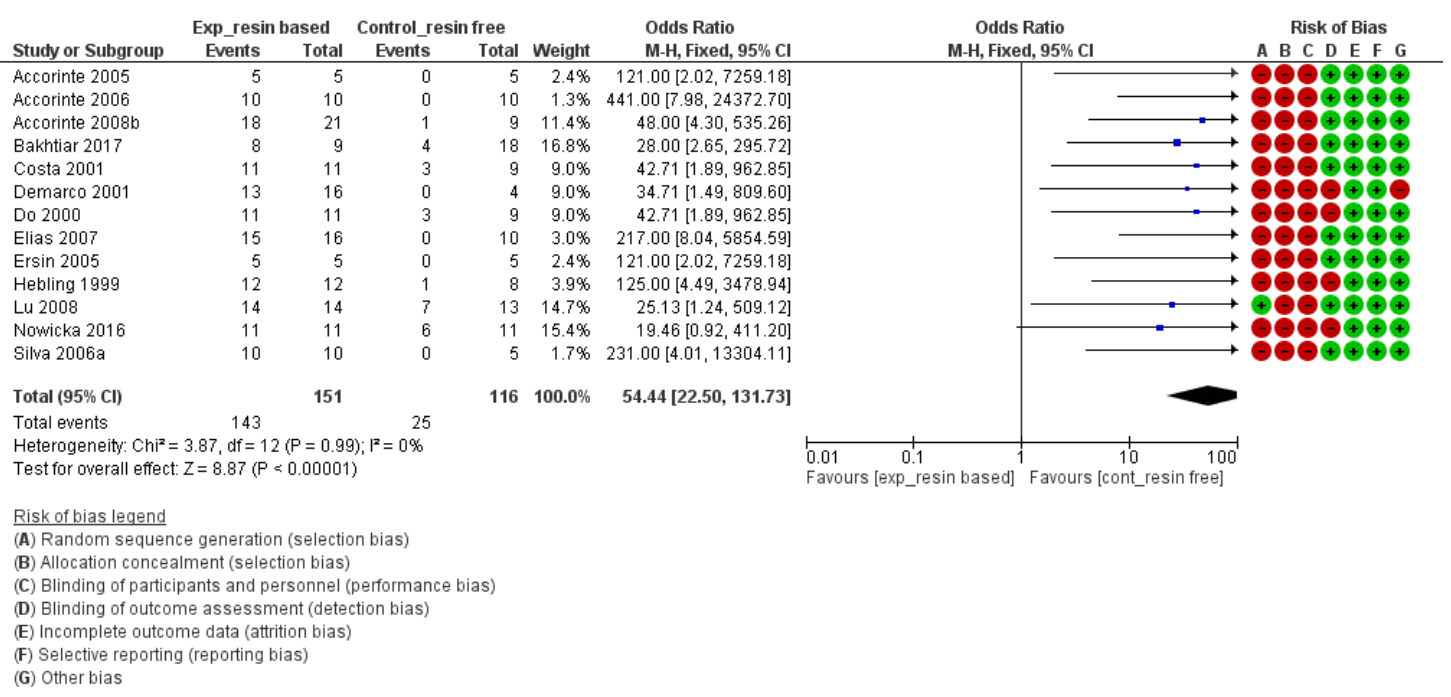

Figure 5. Forest plot and risk of bias of the studies directly comparing the formation of a complete reparative bridge with resin-based and resin-free materials (including pure calcium hydroxide powder, calcium hydroxide cements and hydraulic Calcium-Silicate cements) (odds ratio (95\% CI), random effects). An "event" was considered the lack of a complete hard bridge after 30 days of pulp-capping.

Table 5. Relative risks (95\% CIs) and certainty in the evidence for pulp inflammation at day 30 after direct pulp-capping treatment on human pulp tissue.

\begin{tabular}{|c|c|c|c|c|}
\hline \multirow{2}{*}{ Comparison } & \multicolumn{2}{|c|}{ Direct Comparison } & \multicolumn{2}{|c|}{ Indirect Comparison } \\
\hline & Relative Risk (95\% CIs) & Evidence Level & Relative Risk (95\% CIs) & Evidence Level \\
\hline $\begin{array}{l}\text { Pro-Root MTA vs. } \\
\text { calcium-hydroxide } \\
(\mathrm{CH}) \text { powder }\end{array}$ & No studies available & Not available & No studies available ${ }^{1}$ & Not available \\
\hline $\begin{array}{l}\text { Pro-Root MTA vs. CH cements } \\
\text { (Dycal, Life, Calcipast, ... ) }\end{array}$ & $0.29[0.01,5.79]$ & VERY LOW ${ }^{2 b}$ & $0.39[0.04,3.47]$ & $\mathrm{LOW}^{\mathrm{a}}$ \\
\hline $\begin{array}{c}\text { MTA, Angelus MTA, ... ) vs. } \\
\text { CH powder }\end{array}$ & $3.00[0.14,65.90]^{3}$ & VERY LOW 3 b & $2.67[0.29,24.24]$ & $\mathrm{LOW}^{\mathrm{a}}$ \\
\hline $\begin{array}{l}\text { MTA-like cements vs. } \\
\text { CH cements }\end{array}$ & $0.29[0.01,5.79]$ & VERY LOW ${ }^{b}$ & $0.87[0.16,4.71]$ & $\mathrm{LOW}^{\mathrm{a}}$ \\
\hline $\begin{array}{l}\text { MTA-like cements vs. ALL } \\
\text { CH-based materials (CH } \\
\text { cements + powder) }\end{array}$ & $0.93[0.15,5.79]$ & LOW & $1.17[0.26,5.26]$ & $\mathrm{LOW}^{\mathrm{a}}$ \\
\hline $\begin{array}{l}\mathrm{CH} \text { cements vs. } \mathrm{CH} \text { powder } \\
\text { Calcium-silicate cements }\end{array}$ & No studies available & Not available & $5.41[0.27,108.93]$ & $\mathrm{LOW}^{\mathrm{a}}$ \\
\hline $\begin{array}{l}\text { (Biodentine, iRoot, ... ) vs. } \\
\text { MTA-like cements }\end{array}$ & No studies available ${ }^{4}$ & Not available & No studies available ${ }^{4}$ & Not available \\
\hline $\begin{array}{l}\text { Resin-based vs. } \\
\text { resin-free materials }\end{array}$ & $2.41[0.48,12.03]$ & VERY LOW ${ }^{b}$ & $1.33[0.44,4.04]$ & MODERATE $^{a}$ \\
\hline
\end{tabular}

${ }^{1} 11$ studies available for indirect comparison between Pro-Root MTA (3/11 studies) vs. CH powder (8/11 studies). However, all of them retrieved 0 events (no severe inflammation or necrosis/abscess formation reported). Therefore, quantitative analysis is not possible. ${ }^{2}$ Only 2 studies available for direct comparison between Pro-Root MTA vs. CH cements $[53,100] .{ }^{3}$ Only 1 study available for direct comparison between MTA-like cements vs. CH powder [52]. ${ }^{4}$ No studies available evaluating inflammation of calcium-silicate cements (Biodentine, iRoot, ... ) at 30 days or before. ${ }^{a}$ Level of the evidence downgraded one level due to high risk of bias in all studies and due to imprecision as very few studies are available with very few events. ${ }^{b}$ Level of the evidence very low due to high risk of bias in all studies and due to imprecision as very few studies are available with very few events. 
Table 6. Relative risks (95\% CIs) and certainty in the evidence for pulp inflammation at day 15 after direct pulp-capping treatment on human pulp tissue.

\begin{tabular}{|c|c|c|c|c|}
\hline \multirow{2}{*}{ Comparison } & \multicolumn{2}{|c|}{ Direct Comparison } & \multicolumn{2}{|c|}{ Indirect Comparison } \\
\hline & Relative Risk (95\% CIs) & Evidence Level & Relative Risk (95\% CIs) & Evidence Level \\
\hline $\begin{array}{l}\text { Pro-Root MTA vs. } \\
\text { calcium-hydroxide } \\
(\mathrm{CH}) \text { powder }\end{array}$ & No studies available $^{1}$ & Not available $^{1}$ & No studies available ${ }^{1}$ & Not available $^{1}$ \\
\hline $\begin{array}{l}\text { Pro-Root MTA vs. CH cements } \\
\text { (Dycal, Life, Calcipast, ... ) }\end{array}$ & Not available $^{2}$ & Not available & $0.54[0.02,15.30]$ & VERY LOW ${ }^{a}$ \\
\hline $\begin{array}{l}\text { MTA-like cements (Pro-Root } \\
\text { MTA, Angelus MTA, ... ) vs. } \\
\text { CH powder }\end{array}$ & No studies available ${ }^{3}$ & Not available & No studies available ${ }^{3}$ & Not available ${ }^{3}$ \\
\hline $\begin{array}{l}\text { MTA-like cements vs. } \\
\text { CH cements }\end{array}$ & $0.33[0.02,6.65]^{3}$ & VERY LOW ${ }^{a}$ & Not available ${ }^{3}$ & VERY LOW a \\
\hline $\begin{array}{l}\text { MTA-like cements vs. ALL } \\
\text { CH-based materials (CH } \\
\text { cements + powder) }\end{array}$ & $0.33[0.02,6.65]^{3}$ & VERY LOW a & Not available ${ }^{3}$ & VERY LOW a \\
\hline $\mathrm{CH}$ cements vs. $\mathrm{CH}$ powder & No studies available & Not available & No studies available ${ }^{4}$ & Not available \\
\hline $\begin{array}{l}\text { Calcium-sillcate cements } \\
\text { (Biodentine, iRoot, ...) vs. } \\
\text { MTA-like cements }\end{array}$ & No studies available ${ }^{5}$ & Not available & No studies available ${ }^{5}$ & Not available \\
\hline $\begin{array}{l}\text { Resin-based vs. } \\
\text { resin-free materials }\end{array}$ & No studies available ${ }^{6}$ & Not available & No studies available ${ }^{6}$ & Not available \\
\hline \multicolumn{5}{|c|}{$\begin{array}{l}{ }^{1} \text { Only one study available for Pro-Root MTA (none for CH powder) [103]. }{ }^{2} \text { One study for direct comparison } \\
\text { between Pro-Root MTA and CH cements (Life, Dycal, Calcipast, etc.) at day } 15 \text {. However, it gave } 0 \text { events } \\
\text { (no severe inflammation or necrosis/abscess formation reported). Therefore, quantitative analysis was not possible. } \\
{ }^{3} \text { Only two studies available for MTA-like cements (both of them direct comparison against CH cements, none for } \\
\mathrm{CH} \text { powder) [89,103]. }{ }^{4} \text { Only two studies available, both for CH cements [89,103]. }{ }^{5} \text { No studies available evaluating } \\
\text { inflammation of calcium-silicate cements (Biodentine, iRoot, etc.) at } 30 \text { days or before. }{ }^{6} \text { No studies available } \\
\text { evaluating inflammation of resin-based materials at } 15 \text { days. a Level of the evidence very low due to high risk of } \\
\text { bias in all studies and due to imprecision as very few studies are available with very few events. }\end{array}$} \\
\hline
\end{tabular}

Table 7. Relative risks (95\% CIs) and certainty in the evidence for pulp inflammation up to day 7 after direct pulp-capping treatment on human pulp tissue.

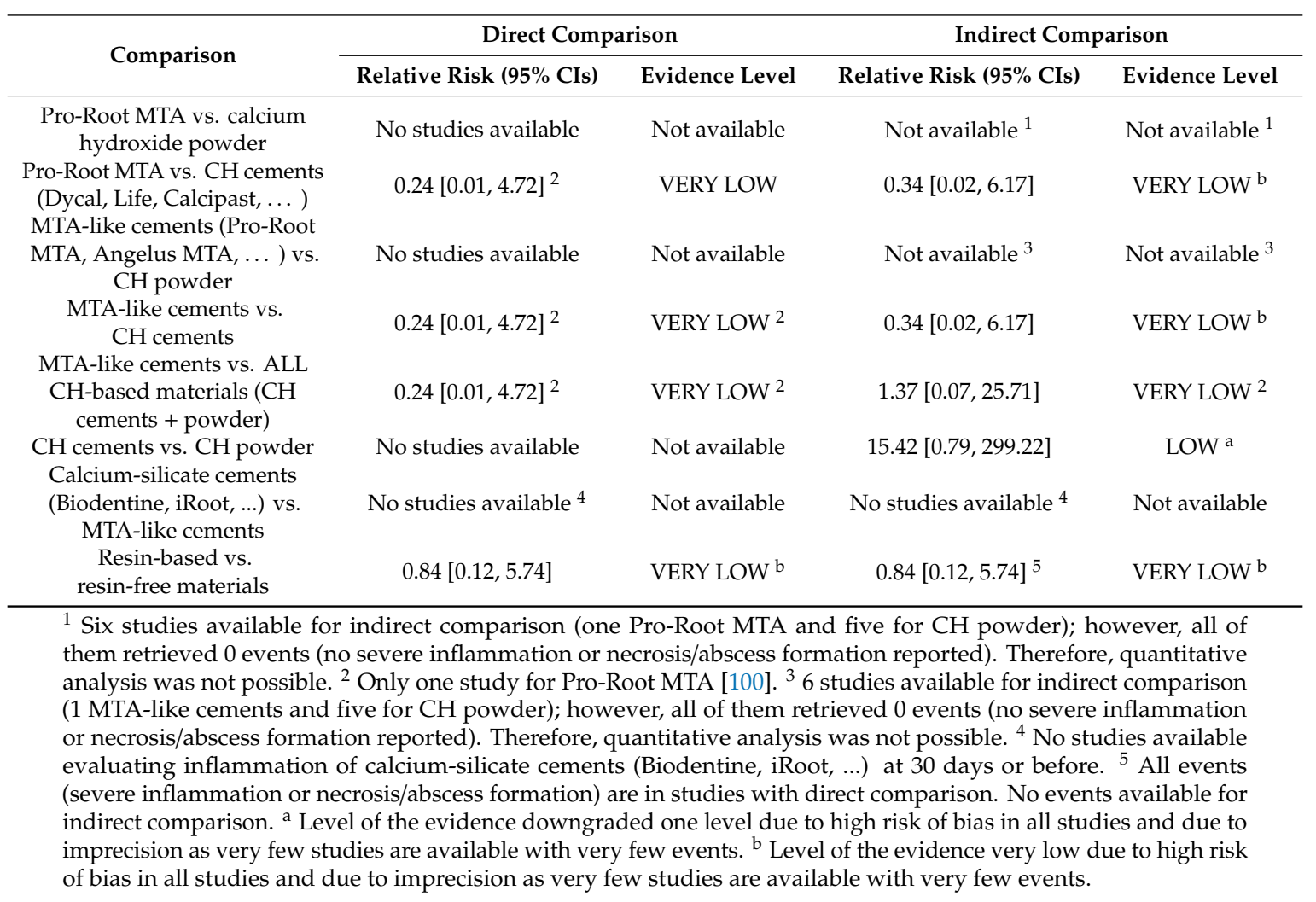


Table 8. Relative risks (95\% CIs) and certainty in the evidence for bridge formation after direct pulp-capping treatment on human pulp tissue.

\begin{tabular}{|c|c|c|c|c|}
\hline \multirow{2}{*}{ Comparison } & \multicolumn{2}{|c|}{ Direct Comparison } & \multicolumn{2}{|c|}{ Indirect Comparison } \\
\hline & Relative Risk (95\% CIs) & Evidence Level & Relative Risk (95\% CIs) & Evidence Level \\
\hline $\begin{array}{l}\text { Pro-Root MTA vs. } \\
\text { Calcium-hydroxide } \\
(\mathrm{CH}) \text { powder }\end{array}$ & No studies available & Not available & $1.64[0.98,2.77]$ & MODERATE $^{a}$ \\
\hline $\begin{array}{c}\text { Pro-Root MTA vs. CH } \\
\text { cements (Dycal, Life, } \\
\text { Calcipast, ... ) }\end{array}$ & $0.39[0.22,0.67]^{* *}$ & HIGH & $0.56[0.42,0.74]^{* * *}$ & HIGH \\
\hline $\begin{array}{l}\text { MTA-like cements (Pro-Root } \\
\text { MTA, Angelus MTA, ... ) vs. } \\
\text { CH powder }\end{array}$ & $1.50[0.66,3.43]^{1}$ & VERY LOW ${ }^{1}$ & $2.02[1.21,3.36]^{* *}$ & MODERATE ${ }^{a}$ \\
\hline $\begin{array}{l}\text { MTA-like cements vs. } \\
\text { CH cements }\end{array}$ & $0.41[0.23,0.73]^{* *}$ & HIGH & $0.59[0.45,0.78]^{* * *}$ & HIGH \\
\hline $\begin{array}{l}\text { MTA-like cements vs. ALL } \\
\text { CH-based materials (CH } \\
\text { cements + powder) }\end{array}$ & $0.66[0.47,0.92] *$ & HIGH & $0.80[0.62,1.03]$ & HIGH \\
\hline $\mathrm{CH}$ cements vs. $\mathrm{CH}$ powder & Not available & Not available & $3.23[2.00,5.20]^{* * *}$ & MODERATE $^{a}$ \\
\hline $\begin{array}{l}\text { Calcium-silicate cements } \\
\text { (Biodentine, iRoot, ...) vs. } \\
\text { MTA-like cements }\end{array}$ & $0.84[0.43,1.65]$ & MODERATE $^{a}$ & $0.81[0.47,1.40]$ & MODERATE $^{a}$ \\
\hline $\begin{array}{c}\text { Biodentine vs. Pro-Root } \\
\text { MTA }\end{array}$ & $0.50[0.04,6.43]$ & VERY LOW $3 \mathrm{~b}$ & $0.86[0.36,2.02]$ & $\mathrm{LOW}^{\mathrm{b}}$ \\
\hline $\begin{array}{l}\text { Calcium-silicate cements vs. } \\
\text { CH powder }\end{array}$ & Not available & Not available & $1.80[0.95,3.40]$ & LOW $a b$ \\
\hline $\begin{array}{l}\text { Calcium-silicate cements vs. } \\
\text { CH cements }\end{array}$ & $0.25[0.10,0.62] * 2$ & VERY LOW ${ }^{2}$ & $0.47[0.15,1.44]$ & LOW $a b$ \\
\hline $\begin{array}{l}\text { Resin-based vs. } \\
\text { resin-free materials }\end{array}$ & $3.69[2.23,6.12]]^{* * *}$ & $\mathrm{HIGH}^{\mathrm{a}}$ & Not performed ${ }^{4}$ & Not performed \\
\hline \multicolumn{5}{|c|}{$\begin{array}{l}{ }^{1} \text { Only one study available for direct comparison between MTA-like cements vs. CH powder [52]. }{ }^{2} \text { Only } 1 \text { study } \\
\text { available for direct comparison between calcium-silicate cements vs. CH cements }[107] .{ }^{3} \text { Only two studies available } \\
\text { for direct comparison between Biodentine vs. Pro-Root MTA [104,106]. }{ }^{4} \text { The direct comparison was strong enough } \\
\text { (13 studies). }{ }^{a} \text { Level of the evidence downgraded one level due to high risk of bias in all studies. }{ }^{b} \text { Level of the } \\
\text { evidence downgraded one level due to high risk of bias in all studies and imprecision. }{ }^{*} \text { The result is statistically } \\
\text { significant }(p<0.05) ;{ }^{* *} \text { The result is statistically significant }(p<0.01) ;{ }^{* * *} \text { The result is statistically significant } \\
(p<0.001 \text { or lower). }\end{array}$} \\
\hline
\end{tabular}

\subsubsection{Heterogeneity Assessment}

The chi-square test and $\mathrm{I}^{2}$ statistic were used to assess heterogeneity. The fixed-effect model is suitable to estimate the typical effect for studies with low heterogeneity $\left(\mathrm{I}^{2}<50 \%\right)$, whereas the random-effects model is used to assess the average distribution for studies with substantial unexplained heterogeneity $\left(\mathrm{I}^{2} \geq 50 \%\right.$ or $\left.p \leq 0.05\right)[109]$.

\subsubsection{Assessment of Publication Bias}

If more than 10 articles were included, publication bias was analysed by visual inspection of funnel plots. An asymmetrical distribution of funnel plot data may suggest the possibility of publication bias [109].

\subsubsection{Summary Measures}

For the parameter "inflammation" of the included in vivo studies, inflammation was noted only when it was scored as severe or when the pulp tissue was defined as necrotic or abscess formation (severe inflammation and/or necrosis/abscess formation were considered as "event" in the Revman 3.5 software). As the parameter "inflammation" is dynamic (it might change over time), it was evaluated separately for different time points (i.e., inflammation up to 7 days, at day 15 and at day 30). Periods longer than 30 days were not taken into account, as inflammation due to material toxicity occurs normally within a short-day range. Inflammation occurring at longer time periods may be due to bacterial infiltration or trauma. 
To evaluate the parameter "bridge formation" of the included in vivo studies, only the presence of a complete bridge was taken into account. When the bridge was incomplete or not present, this was considered as an "event" in the Revman 3.5 software.

The results were expressed as the odds ratios and 95\% CIs (the "relative risk" (RR) was presented in Tables 5-8 for the sake of simplicity in the interpretation of the results). When possible, heterogeneity of the studies and bias publication were also recorded. As many materials have been tested over time and no direct comparison was always available between them, an indirect meta-analysis was performed. Moreover, a network meta-analysis graph was made for all the available studies and their respective interactions (Figure 6).

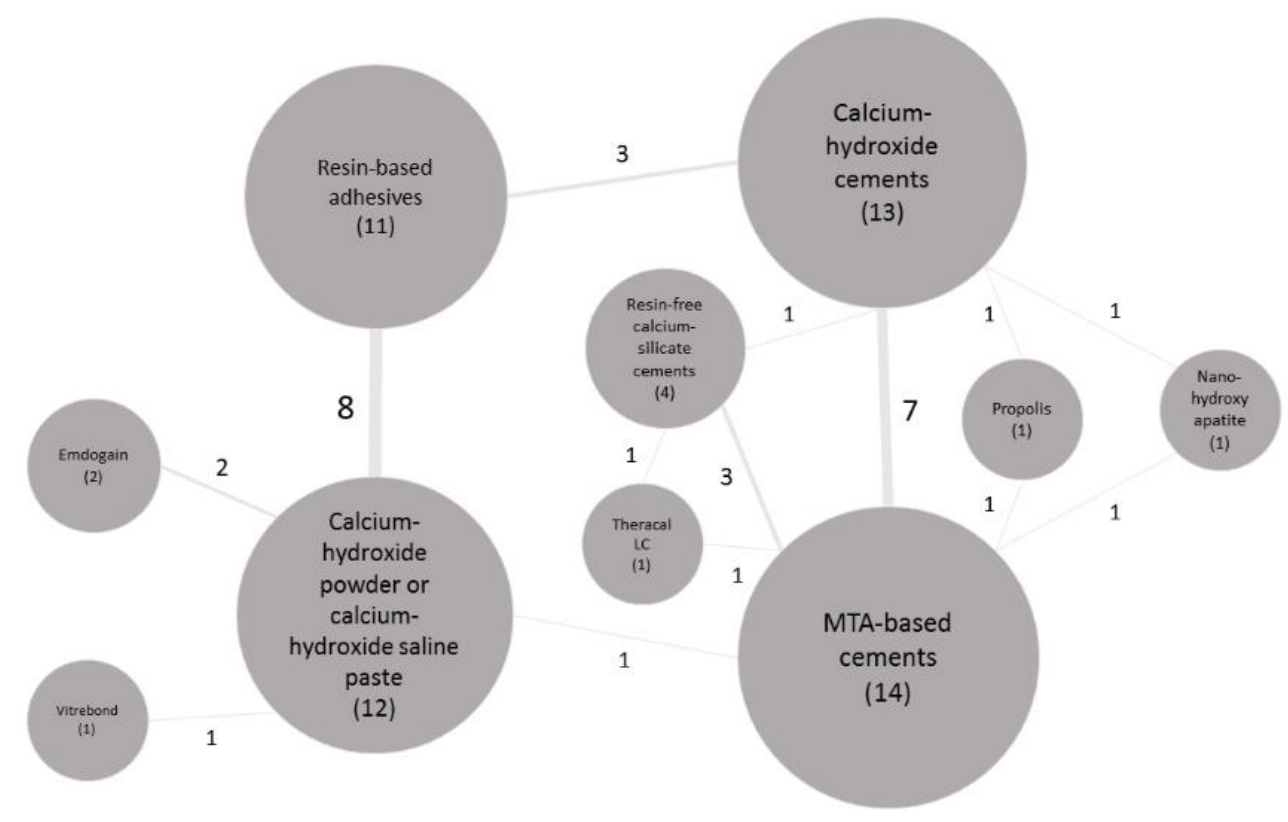

Figure 6. Schematic representation (network) of the interaction among the in vivo studies $(\mathrm{n}=30)$. The balls (nodes) represent the materials and the times each material was studied. The line thickness and the number connected to the lines (edges), linking two materials, represent the frequency of interactions between them.

\subsection{Statistical Analysis}

We conducted a meta-analysis to obtain estimates of the relative effectiveness of all interventions on the primary outcome by combining direct and indirect evidence using a fixed-effects or random-effects model.

\subsection{Risk of Bias Across Studies (Certainty in the Evidence)}

The quality of the evidence was assessed using the "Grading of Recommendations Assessment, Development and Evaluation" (GRADE) approach at the outcome level for each comparison between interventions [111]. The certainty in the evidence can be high, moderate, low or very low. When the certainty is derived from direct comparisons, randomized controlled trials provide high-certainty evidence. However, some issuese such as serious risk of bias, imprecision, inconsistency or publication bias can reduce the certainty [111].

\section{Results}

\subsection{Search Results and Study Selection}

The PRISMA flowchart with the search results and the study selection process can be seen in Figure 1. The PubMed, EMBASE and Web of Science searchs provided 6490, 4369 and 5682 results, 
respectively. The total sum of 16,541 articles was stored in a reference manager (Endnote X9, Clarivate Analytics). Twelve results found by hand searching through the reference lists of the articles and from other sources were added. Duplicates were removed manually with Endnote X9 (Clarivate Anaytics) reference manager, resulting in 10,469 unique articles. From these 10,469 studies, 1536 were excluded because they were published before 1993 (date of the first publication on MTA [15]). From the remaining 8933 articles, 7628 and 1117 articles were excluded as being non-relevant by screening the titles and abstracts, respectively. Finally, 188 articles were eligible for full-text screening (Figure 1). The year of publication ranged from 1946 to 2020 (later reduced to 1993-2020). The selection process is detailed in the PRISMA flow chart (Figure 1). Two in vivo studies that met de inclusion criteria were finally not included (after contacting the authors) in the qualitative/quantitative analysis because the data had been partially used in previous studies (Figure 1) [112,113]. Finally, 56 articles were included in the analysis, 26 involving in vitro studies and 30 in vivo studies. The reasons for exclusion are listed in Figure 1 and a full list of excluded articles (with reason for exclusion) is provided in Supplementary Table S1 (Table S1). Moreover, these 30 in vivo studies were included for quantitative meta-analysis, among which there were 10 controlled clinical trials (CCT) and 20 randomized controlled trials (RCT). The sample sizes of these studies were $n=355$ (premolars) for immature permanent teeth and $n=657$ for mature permanent teeth ( 301 molars and 356 premolars) for a total of 1012 teeth in all 30 included in vivo studies.

\subsection{Study Characteristics}

The characteristics of the included in vitro and in vivo studies are listed in Tables $2-4$. The in vivo studies were subdivided depending on whether they included mature or immature permanent teeth (Tables 3 and 4, respectively).

Risk of Bias within Studies

The risk of bias of the in vivo studies is presented along with the meta-analysis (forest plots; Figures 2-5) and was determined following the Cochrane recommendations using the software Revman 5.3 (Cochrane). In general, except for two studies [85,100], most of the included in vivo studies were deemed as having high risk of bias. The most frequent types of bias were selection and performance biases, as in most studies no "random sequence generation" nor "allocation concealment" were described. It is true that "blinding of participants and personnel" is difficult to achieve as the materials have different appearances. Therefore, when this was the only risk of bias present, we did not consider it a high bias risk. However, this was the case in only two out of 30 studies.

\subsection{Synthesis of Results}

The results for each individual in vitro and in vivo study are presented in Tables 2-4, respectively. For the in vivo studies, a quantitative meta-analytical analysis was performed when possible (Tables 5-8). By preference, the meta-analysis was conducted using studies where direct comparison was evaluated. However, when direct comparison was not available (or not enough), an indirect evaluation was performed. The two parameters quantitatively analysed in vivo were: (1) occurrence of severe inflammation (or necrosis/abscess formation) in the pulp tissue up to a period of 30 days (Tables 5-7), and (2) formation of a complete bridge between the material and the pulp tissue after 30 or more days (Table 8).

\subsubsection{Qualitative Analysis of In Vitro Studies}

The qualitative analysis of the included in vitro studies showed that the materials that were studied the most in contact with human dental pulp cells of primary origin were the hydraulic calcium-silicate cements (hCSCs), Pro-Root MTA (Dentsply-Sirona; 9/26 studies) and Biodentine (Septodont; 7/26 articles) (Table 2). These were followed by MTA-Angelus (Angelus, Londrina, Brazil; 5/26 studies), the calcium-hydroxide ( $\mathrm{CH}$ ) cement Dycal (Dentsply-Sirona, Konstanz, Germany; 
5/26 studies), the resin-free hCSC iRoot BP (Innovative Bioceramix; 4/26 studies), the resin-based calcium-silicate cement (Rb-CSC) Theracal LC (Bisco; 4/26 studies) and Portland cement (Ssangyong, Seoul, Korea; 4/26 studies). The other included materials were tested in three or less studies (Table 2). From the analysis, in general hCSCs (Pro-Root MTA, Dentsply-Sirona; Biodentine, Septodont; MTA-Angelus, Angelus; iRoot BP, Innovative Bioceramix; Portland cement, Ssangyong) were found to be non-cytotoxic when in (in)direct contact with human dental pulp cells, while they also exhibited bioactivity (migration, proliferation, mineralization capacities) towards human tooth pulp cells they were exposed to $[28,59,61-63,65-68,70,74,77,80]$. Moreover, the CH cement Dycal (Dentsply-Sirona) was in general deemed cytotoxic (in some studies cytotoxicity could not be tested because most of the cells died in contact with this calcium-hydroxide cement), and when directly compared, Dycal (Dentsply-Sirona) was more cytotoxic than the hCSCs tested $[58,59,66,80]$. The Rb-CSC Theracal LC (Bisco) seemed not to be as cytotoxic as Dycal (Dentsply-Sirona) [69], but when compared to hCSCs, contradictory results were reported as some authors showed similar results with both materials [70], while other authors showed more cytotoxicity and less bioactivity with Theracal LC (Bisco) [28,62].

3.3.2. Meta-Analysis of the Effect of Pulp-Capping Agents on the Inflammatory Reaction Induced in Human Pulp Tissue

Inflammatory Reaction at Day 30

The inflammatory reaction induced by the different materials at day 30 is shown in Table 5 . No significant difference was found in any of the direct or indirect comparisons/combinations tested. However, the quality/certainty of the evidence was very low or low following the GRADE recommendations. This was mostly due to the lack of studies directly comparing materials or the few studies available.

The materials that were tested the most were Pro-Root MTA (Dentsply-Sirona) and CH powder/saline-11 and eight studies, respectively. For these two materials, severe inflammation or necrosis/abscess formation were not reported in any of the included studies. Other studies investigated CH cements, such as Dycal (Dentsply-Sirona) or Life (Kerr, Orange, CA, USA); other MTA-like products, such as MTA-Angelus (Angelus); and resin-based adhesives.

Inflammatory Reaction at Day 15

The inflammation induced by the different materials at day 15 is shown in Table 6 . No significant difference was found in any of the direct or indirect comparisons/combinations tested. However, not many studies were found in this time-period category and therefore not many comparisons could be included. In this case, the quality/certainty of the evidence was very low or low following the GRADE recommendations. This is mostly due to the lack of studies directly comparing materials or the few studies available.

Inflammatory Reaction up to Day 7

The inflammatory reaction induced by the different materials at day 7 is shown in Table 7 . No significant difference was found in any of the direct or indirect comparisons/combinations tested. However, the quality/certainty of the evidence was very low or low following the GRADE recommendations. Likewise, as explained above for the 15 and 30-day periods, this must mostly be attributed to the lack of studies directly comparing materials or the few studies available.

Meta-Analysis of the Effect of Pulp-Capping Materials on Hard-Tissue Formation upon Capping Exposed Human Pulp Tissue

The ability of the each pulp-capping agent to induce dentin-bridge formation is shown in Table 8 . Some comparisons between materials did reveal significant differences (Table 8 ). The most frequently tested materials were: (1) resin-free MTA-like cements within 14 studies [12 studies tested Pro-Root 
MTA (Dentsply-Sirona) and two studies Angelus-MTA (Angelus)], (2) calcium-hydroxide cements within 13 studies [nine studies tested Dycal (Dentsply-Sirona), three studies Life (Kerr), one study Calcimed (Cerkamed, Stalowa Wola, Poland) and one study Multi-Cal Liner (Pulpdent, Watertown, MA, USA)], (3) pure calcium-hydroxide powder/saline paste within 12 studies and (4) resin-based adhesives within 11 studies [three studies tested Scotchbond Multipurpose (3M, Seefeld, Germany), three studies Clearfil SE Bond (Kuraray Noritake, Tokyo, Japan), three studies Clearfil Liner Bond 2 (Kuraray Noritake, Tokyo, Japan), one study Single Bond (3M, Seefeld, Germany), one study Single Bond Universal (3M, Seefeld, Germany), one study All-Bond 2 (Bisco) and one study Prime\&Bond NT 2.1 (Dentsply-Sirona, Konstanz, Germany)]. To have a better overview of how frequently the different capping agents were tested and their interactions, a graphic network analysis is shown in Figure 6.

The materials that more often induced dentin-bridge formation were calcium-hydroxide powder/saline and Pro-Root MTA (Dentsply-Sirona). Unfortunately, the two cements were not directly compared and the reported results originate from indirect comparisons (Table 8). However, when both materials were indirectly compared (Table 8), no significant difference in dentin-bridge formation was found (Relative risk $(95 \% \mathrm{CI})=1.64(0.98,2.77))$, although the difference was statistically borderline non-significant in favour of calcium-hydroxide powder/saline $(p=0.06)$ (Figure 2$)$. The quality/certainty of the evidence was ranked as moderate, since the data were gathered from many different studies conducted by many different authors (Table 8 and Figure 2). When MTA-Angelus (Angelus) was also taken into account, the difference between the materials was significant in favour of calcium-hydroxide powder/saline (relative risk $(95 \% \mathrm{CI})=2.24(1.19,4.20 ; p=0.007)$; one study that directly compared materials was then included [52] (Table 8).

On the other hand, calcium-hydroxide cements, such as Dycal (Dentsply-Sirona) and Life (Kerr), performed significantly worse than calcium-hydroxide powder/saline (Relative risk $(95 \% \mathrm{CI})=3.23$ $(2.00,5.20), p<0.00001)$ and MTA-like cements (relative risk $(95 \% \mathrm{CI})=0.41(0.23,0.73), p<0.0001)$ (Figures 3 and 4, respectively).

When calcium-hydroxide cements were indirectly compared to hydraulic calcium-silicate cements (hCSCs), such as Biodentine (Septodont) and iRoot BP (Innovative Bioceramix, Vancouver, Canada), no significant difference was found in favour of hCSCs (relative risk $(95 \% \mathrm{CI})=0.47(0.15,1.44), p=0.12$ ) (Table 8$)$. The difference was significant in favour of hCSC $(p<0.001)$ when the materials were directly compared. However, we found only one study where these two materials were directly compared, because of which this finding should be taken with caution [107]. The quality of evidence varied among the different comparisons from high (MTA-like materials vs. calcium-hydroxide $(\mathrm{CH})$ cements), to moderate ( $\mathrm{CH}$ cements vs. $\mathrm{CH}$ powder/saline) to low (hCSCs vs. $\mathrm{CH}$ cements) depending on the amount and quality of studies available.

Furthermore, the comparison between resin-based vs. resin-free materials was significantly different in favour of the resin-free materials (relative risk $(95 \% \mathrm{CI})=3.69(2.23,6.12), p<0.0001)$ (Table 8 and Figure 5). The result was significantly different independently of which resin-free material was used (CH powder, $\mathrm{CH}$ cements, resin-free MTA-like cements or resin-free hCSCs).

\subsection{Risk of Bias Across Studies}

The risk of bias across studies (interpreted as the certainty of quality of evidence) was analysed using the GRADE recommendation. For most of the comparisons done between material categories for the parameter "Inflammatory reaction," the certainty/quality in evidence was mostly evaluated as "very low" or "low." This must mostly be ascribed to high risk of bias in individual studies but also to the few articles present in most categories increasing the imprecision and inaccuracy levels (Tables 5-7).

For the parameter "reparative bridge formation," after pulp capping with different materials, most comparisons were categorized as of "moderate" or "high" quality. In this case, the number of articles that directly compared materials was higher than for the "inflammation" parameter. Moreover, the total number of cases available was also high, reducing the total imprecision and inaccuracy levels (Table 8). 


\section{Discussion}

\subsection{Summary of Results}

The results of this systematic review showed that cell viability and bioactivity of the pulp-capping materials exposed to human dental pulp cells in vitro differ among the materials tested (Table 2). Therefore, the first null-hypothesis that there is no difference among the materials tested, was rejected.

Moreover, the outcomes of the meta-analysis regarding in vivo inflammatory effect of the different pulp-capping agents did not reveal differences among the materials tested (Tables 5-7). Therefore, we failed to reject the second null-hypothesis.

In vivo, the formation of a complete hard-tissue bridge in healthy human teeth after exposure and subsequent pulp capping varied as a function of the materials used (Table 8). In this way, the third null-hypothesis that there is no difference in hard-tissue formation among the materials tested, was also rejected.

The main conditions for a successful vital pulp therapy are: (1) a healthy patient with a good healing capacity, (2) a pulp-tissue environment free of bacteria and (3) a biocompatible material $[45,114-116]$. Of these three characteristics, we were interested in studying the effect of biocompatibility of the different pulp-capping materials on human pulp tissue. However, biocompatibility is a broad term requiring both in vitro and in vivo tests to be conducted. This is the reason why we included studies evaluating cytotoxicity and bioactivity of materials by exposing human pulp tissue in vitro and in vivo to these materials. There are also many studies evaluating biocompatibility towards pulp tissue in animals. However, it has been shown that there might be differences in reaction of pulp tissue from animals as compared to humans due to different metabolisms and immune system responses [117,118]. Therefore, because of the relatively high availability of human studies, we decided to include only studies performed on human dental pulp cells or human teeth.

The main reason for failure after vital pulp therapy is (re)infection of pulp tissue by bacteria $[9,10,45,116,119]$. Therefore, in order to selectively evaluate the effectiveness of different pulp-capping agents, we included only studies that performed vital pulp therapy on sound teeth from healthy volunteers using a strict aseptic environment (i.e., rubber dam isolation). By doing so, we intended to avoid cofounding factors like environmental contamination or lack of healing capacity of patients.

\subsubsection{Systematic Review of In Vitro Studies}

The systematic review of the included in vitro studies showed that not much research has been done evaluating the direct effect of dental adhesives and resin composites on human dental pulp cells. Only two out of 26 studies (one for adhesives [28] and one for resin composites [78]) (Table 2) met the inclusion criteria (Table 1). The reason for this might be that the dental scientific community [based mostly on the results from in vitro studies and histology involving animal and human teeth (Tables 3-5)] has accepted that resins and monomers eluted from adhesives and composites are toxic for pulp cells $[29,81,83,84,86,92,93,97,98,105,120-125]$. However, this is in contradiction with the desire and increasing tendency from industry and researchers to develop resin-based pulp-capping materials, which are more user and patient friendly. Some examples of this kind of material are the resin-based calcium-hydroxide cements Prisma VLC (Dentsply-Sirona), Calcimol (Voco, Cuxhave, Germany) and Lime-Lite (Dentsply-Sirona), and the resin-modified calcium-silicate materials Theracal LC (Bisco) and Biocal Cap (Harvard).

It is difficult to make much out of the results of in vitro studies because most of them each use a different method to prepare the materials or to expose the cells. Moreover, not all of them refer to the ISO-standards (only 10/26 studies explicitly mentioned the use of ISO-standards) and the ISO-standards have been changing over time. This is important because if the data cannot be compared, the results are difficult to interpret and extrapolate. Furthermore, companies and researchers have to adhere to 
ISO-standards to develop/evaluate their materials. If the experiments are not done following these recommendations, the results might be less applicable.

Another particular issue with research involving hCSCs is that most researchers used "set" materials to conduct their experiments (only 2/26 included in vitro studies used "freshly-mixed" cements in their experimental protocol; Table 2) $[66,76]$. On the one hand, a protocol involving "set" materials is very useful, as it allows an easier set-up and more standardization of the experiments. However, care should be taken when designing the protocols, as hCSCs are very sensitive to drought (they need water to set and achieve their physio-mechanical properties), and, more importantly, in the clinic they are brought into contact with the pulp tissue when they are still setting ("freshly mixed"). Therefore, the use of "freshly-mixed" cements has a benefit in the case of these pulp-capping agents.

\subsubsection{Systematic Review and Meta-Analysis of In Vivo Studies}

\section{Inflammatory Reaction}

From the analysis of the inflammatory reactions of human pulp tissue exposed to the biomaterials tested, we may conclude that there exists no difference in inflammatory reaction induced by the materials tested (Tables 5-7). However, we have to be cautious because evaluating inflammatory reaction can be very subjective. This is the reason why we decided to count as "events" only very strong inflammatory reactions, such as severe inflammation or necrosis/abscess formation. Even though in most of studies an independent examiner was selected to avoid risk of bias, the difference between slight and moderate inflammatory reaction is very subjective. Severe inflammation or necrosis are in principle more objective outcomes. We should also bear in mind that hard-tissue histology, particularly tooth histology, is a difficult technique, even for pathologists (as they are not often confronted with hard tissues such as teeth). Much methodological experience is needed before quality sections can be prepared and trustable information gathered. It may happen that artefacts caused by incorrect fixation or induced during demineralization and cutting procedures are confused with pulp-tissue damage. Moreover, in most studies a limited number of sections are obtained. This is not ideal, as pulp tissue may appear normal in one part but inflamed in another part. Therefore, it is recommended that the whole pulp tissue is cut and sections from different areas are stained and analysed to guarantee representativeness [126].

\section{Complete Reparative Bridge Formation}

For the in vivo quantitative analysis, different comparisons were conducted. Comparison between resin-based and resin-free materials clearly elicited that the latter produce more frequently complete dentin bridges than the former $(p<0.001$; Table 8 and Figure 5). Comparison of Pro-Root MTA (Dentsply-Sirona) with calcium hydroxide $(\mathrm{CH})$ yielded contradictory results. When we analysed studies comparing pure $\mathrm{CH}$ powder with Pro-Root MTA (Dentsply-Sirona), pure $\mathrm{CH}$ powder produced more frequently complete bridges than Pro-Root MTA (Dentsply-Sirona), although no statistically significant difference was recorded $(p=0.06$; odds ratio $(95 \% \mathrm{CI})=1.85(0.98,3.49))$ (Figure 2). However, when we evaluated studies comparing $\mathrm{CH}$ cements, such as Dycal (Dentsply-Sirona) vs. Pro-Root MTA (Dentsply-Sirona), a significant difference in the formation of complete bridges was found in favour of MTA ( $p<0.01$; Table 8 and Figure 3). This is relevant, especially in places were the high cost and/or lack of a national insurance system make it difficult for clinicians and patients to afford materials like Biodentine (Septodont) or Pro-Root MTA (Dentsply-Sirona). Moreover, many researchers and clinicians consider CH cements, such as Dycal (Dentsply-Sirona) and Life (Kerr), to be the same as or similar to as pure $\mathrm{CH}$ powder, and therefore, as gold-standard materials for vital pulp therapy [69]. This has led to many clinical trials evaluating pulp-capping treatment with $\mathrm{CH}$ cements serving as control ("gold-standard") materials, with disastrous results $[7,16,17,127,128]$. Therefore, as previous research had already highlighted, and considering the results of this meta-analysis, pure $\mathrm{CH}$ powder and $\mathrm{CH}$ cements should not be considered to be the same $[52,129,130]$. Even though the quantity/quality of 
mineral bridge formation seems higher when using resin-free hCSCs [131], pure $\mathrm{CH}$ powder is a very cheap alternative with excellent efficiency for vital pulp therapy. Its efficacy has been backed up with almost 100 years of scientific evidence behind it, including in vitro, ex vivo and in vivo studies on animal and human teeth [126,132-134].

\subsection{Comparison with Previous Studies and Limitations}

Recently, there have been many review articles about this topic [48-51,135]. However, some limitations of these reviews were that, (1) they focused on the comparison between $\mathrm{CH}$ compounds and MTA [50]; (2) they only included in their analyses studies with a direct comparison between the materials tested [48,49] or (3) they only compared in vitro articles [51,135]. In our systematic review, we wanted to go further, and we included all types of pulp-capping materials and, both in vitro and in vivo studies. Moreover, we also did an indirect comparison between the different materials tested, similarly to a network meta-analysis [136]. By doing so, we were able to include a comparison between pure $\mathrm{CH}$ powder and Pro-root MTA (Dentsply-Sirona), as there has been, to our knowledge, no research performed in human teeth comparing both materials directly $[112,137]$.

Some of the limitations of this study are the narrow scope of the review by including only in vitro studies performed on human dental pulp cells from primary sources and in vivo studies performed on healthy permanent human teeth using strict aseptic criteria. However, we think that there have been other recent reviews studying the effect of these type of materials on other cell lines (i.e., human dental (stem) cells from the apical papilla (SCAPs) or (stem) cells from human exfoliated deciduous teeth (SHEDs)) and also using animal cell lines/tissue or teeth $[48,135]$. Other limitations may be the limited timespan of the review, as we took into consideration only articles from 1993, and the short-term follow-up of the in vivo studies. Regarding the timespan of the review, we did so because the first hydraulic calcium-silicate cement (Pro-Root MTA, Dentsply-Sirona) was developed in 1993 and the first article was published in 1995. Moreover, the use of human dental pulp cells of primary origin became popular from the year 2000 and on, after publication that stem cells could be harvested from human teeth [138-140]. In relation to the short-term follow-up of the in vivo studies, we wanted to evaluate the histological characteristics of the inflammatory reaction and the reparative bridge formation after exposure with different pulp-capping agents. In the long-term, other factors like the health status of the patient or the quality of the coronal restoration play important roles. Therefore, in order to exclude or minimize these co-founding factors, we wanted to focus solely in the (short-term) histological features of the healing after pulp-capping. However, care should be taken when interpreting the results of this systematic review and meta-analysis, as some articles studying the effects of dental materials on human cells or teeth might have been left aside for those reasons.

\section{Conclusions and Recommendations for Future Research}

In conclusion, we want to stress that materials such as pure calcium-hydroxide $(\mathrm{CH})$ powder and Pro-Root MTA (Dentsply-Sirona) have shown excellent biocompatibility in vitro and in vivo when tested on human cells and teeth. Their use after many years of research and clinical experience seems safe and proven for vital pulp therapy in healthy individuals given that an aseptic environment (rubber dam isolation) is maintained. More recently introduced hCSCs like Biodentine (Septodont) and iRoot BP (Innovative Bioceramix) showed promising results, but more studies are necessary to confirm their suitability. However, in general, all these hCSCs have many disadvantages, such as long setting times, lack of bonding capacity to tooth structures, high solubility and high risk of discoloration, which make them difficult to handle and less user and patient friendly.

Newer, setting-on-command materials, such as resin-based calcium-silicate cements, are therefore highly desirable. Nevertheless, before these innovative materials can be used in patients, improved bioactivity and biocompatibility are needed and mandatory. Moreover, for these materials to be successful in the clinical practice, better bonding capacity to hard dental tissues and enhanced physio-mechanical properties are also needed. On the one hand, by having a strong bonding ability 
to dentin, better sealing ability and reduced long-term bacterial leakage is expected. On the other hand, increased physio-mechanical properties may ensure that these materials can be used also as definitive restorations. We highly encourage clinicians not to use new materials in patients until sufficient scientific evidence has been provided. Even minor changes in composition may have drastic consequences in the clinical outcomes of our treatments.

Supplementary Materials: The following are available online at http://www.mdpi.com/1996-1944/13/12/2670/s1. Figure S1: Details of the PubMed electronic search strategy, Figure S2: Details of the EMBASE electronic search strategy, Figure S3: Details of the WEB OF SCIENCE electronic search strategy, Table S1. Excluded full-text in vivo studies and the reasons for exclusion.

Author Contributions: M.S.P. and X.L. contributed to the conception of the work and acquisition, analysis and interpretation of data; Moreover, they drafted the work and have approved the submitted version. K.Y., K.V.L. and B.V.M. contributed to the conception of the work and interpretation of the data. Moreover, they drafted the manuscript, substantively revised it and approved the submitted version. All authors have read and agreed to the published version of the manuscript.

Funding: MSP is appointed as PhD fellow ("Aspirant") of the Research Foundation-Flanders (FWO; 11B4320N). This research was supported by the FWO research grant G.0893.15.

Acknowledgments: The authors wish to thank Magdalena Jans, Thomas Vandendriessche, Kristel Paque and Krizia Tuand, biomedical reference librarians of the KU Leuven library "2Bergen-learning Centre Désiré Collen" (KU Leuven, Leuven, Belgium), for their help in conducting the systematic literature search. We also want to thank all the authors contacted by e-mail or Researchgate for their precious time and kind collaboration, thereby providing full details about their research papers.

Conflicts of Interest: The authors declare no conflict of interest.

\section{Abbreviations}

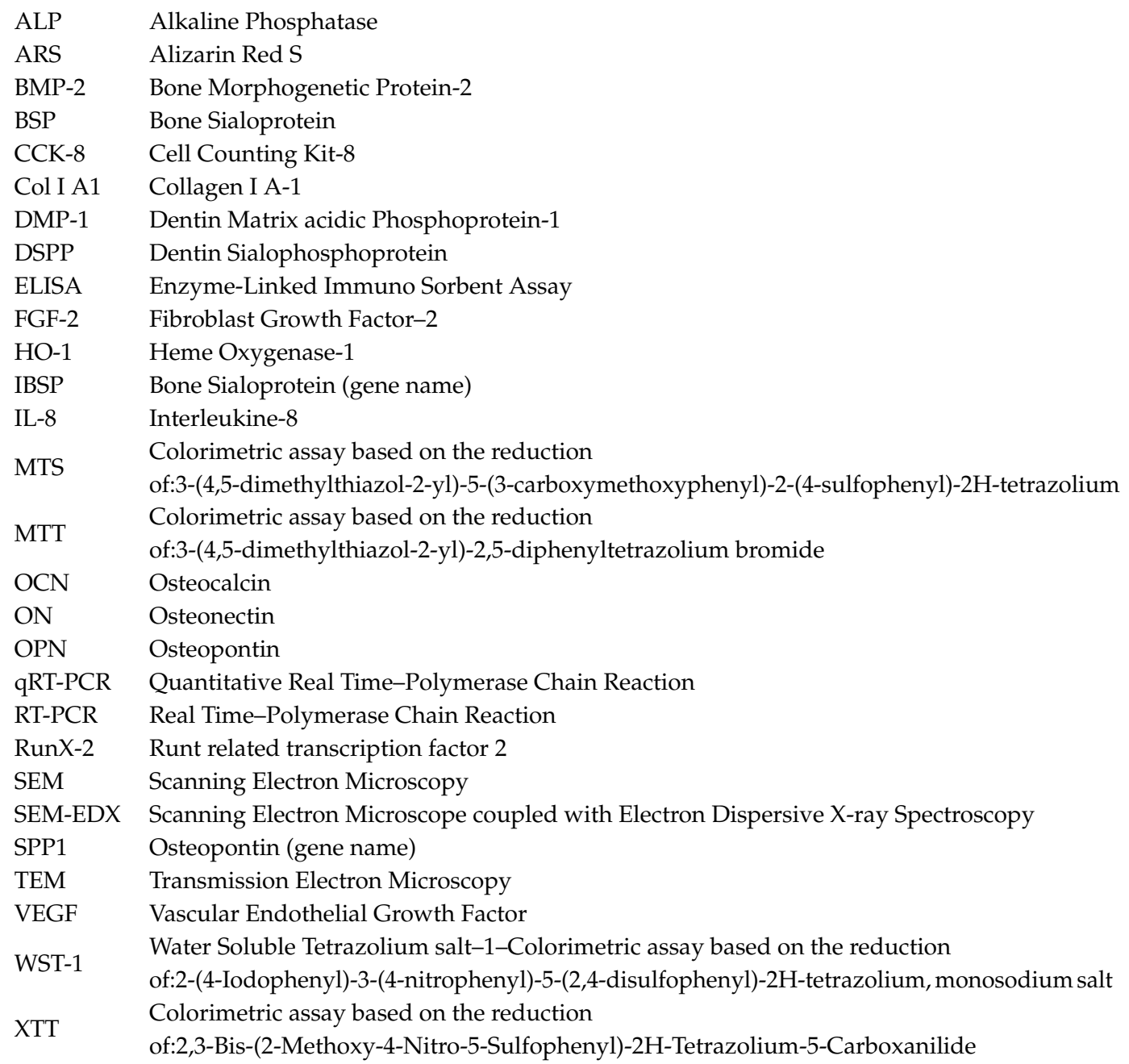




\section{References}

1. Pulp Capping and Pulpotomy Agents. 2020. Available online: https://www.ncbi.nlm.nih.gov/mesh?Db= mesh\&Term=\%22Dental\%20Pulp\%20Capping\%20\%22[MESH] (accessed on 15 May 2020).

2. Olsson, H.; Petersson, K.; Rohlin, M. Formation of a Hard Tissue Barrier after Pulp Cappings in Humans. A Systematic Review. Int. Endod. J. 2006, 39, 429-442. [CrossRef] [PubMed]

3. Schröder, U. Effects of Calcium Hydroxide-Containing Pulp-Capping Agents on Pulp Cell Migration, Proliferation, and Differentiation. J. Dent. Res. 1985, 64, 541-548. [CrossRef] [PubMed]

4. About, I. Dentin Regeneration in Vitro: The Pivotal Role of Supportive Cells. Adv. Dent. Res. 2011, 23, 320-324. [CrossRef] [PubMed]

5. Ricketts, D.; Lamont, T.; Innes, N.P.T.; Kidd, E.; Clarkson, J.E. Operative Caries Management in Adults and Children. Cochrane Database Syst. Rev. 2013, 3, CD003808. [CrossRef]

6. Schwendicke, F.; Frencken, J.E.; Bjørndal, L.; Maltz, M.; Manton, D.J.; Ricketts, D.; van Landuyt, K.; Banerjee, A.; Campus, G.; Doméjean, S.; et al. Managing Carious Lesions: Consensus Recommendations on Carious Tissue Removal. Adv. Dent. Res. 2016, 28, 58-67. [CrossRef]

7. Bjorndal, L.; Fransson, H.; Bruun, G.; Markvart, M.; Kjaeldgaard, M.; Nasman, P.; Hedenbjork-Lager, A.; Dige, I.; Thordrup, M. Randomized Clinical Trials on Deep Carious Lesions: 5-Year Follow-Up. J. Dent. Res. 2017, 96, 747-753. [CrossRef]

8. Dummer, P.M.H.; Franco, V.; Gambarini, G.; Orstavik, D.; Tjaderhane, L.; Whitworth, J.; Duncan, H.F.; Galler, K.M.; Tomson, P.L.; Simon, S.; et al. European Society of Endodontology Position Statement: Management of Deep Caries and the Exposed Pulp. Int. Endod. J. 2019, 52, 923-934.

9. Ricucci, D.; Loghin, S.; Siqueira, J.F., Jr. Correlation between Clinical and Histologic Pulp Diagnoses. J. Endod. 2014, 40, 1932-1939. [CrossRef]

10. Ricucci, D.; Loghin, S.; Lin, L.M.; Spangberg, L.S.; Tay, F.R. Is Hard Tissue Formation in the Dental Pulp after the Death of the Primary Odontoblasts a Regenerative or a Reparative Process? J. Dent. 2014, 42, 1156-1170. [CrossRef]

11. Taha, N.A.; Khazali, M.A. Partial Pulpotomy in Mature Permanent Teeth with Clinical Signs Indicative of Irreversible Pulpitis: A Randomized Clinical Trial. J. Endod. 2017, 43, 1417-1421. [CrossRef]

12. Schwendicke, F.; Stotpe, M. Direct Pulp Capping after a Carious Exposure Versus Root Canal Treatment: A Cost-Effectiveness Analysis. J. Endod. 2014, 40, 1764-1770. [CrossRef]

13. Darvell, B.W.; Wu, R.C.T. Mta-an Hydraulic Silicate Cement: Review Update and Setting Reaction. Dent. Mater. 2011, 27, 407-422. [CrossRef]

14. Prati, C.; Gandolfi, M.G. Calcium Silicate Bioactive Cements: Biological Perspectives and Clinical Applications. Dent. Mater. 2015, 31, 351-370. [CrossRef]

15. Lee, S.J.; Monsef, M.; Torabinejad, M. Sealing Ability of a Mineral Trioxide Aggregate for Repair of Lateral Root Perforations. J. Endod. 1993, 19, 541-544. [CrossRef]

16. Hilton, T.J.; Ferracane, J.L.; Mancl, L. Comparison of Caoh with Mta for Direct Pulp Capping: A Pbrn Randomized Clinical Trial. J. Dent. Res. 2013, 92 (Suppl. S7), 16s-22s. [CrossRef]

17. Mente, J.; Hufnagel, S.; Leo, M.; Michel, A.; Gehrig, H.; Panagidis, D.; Saure, D.; Pfefferle, T. Treatment Outcome of Mineral Trioxide Aggregate or Calcium Hydroxide Direct Pulp Capping: Long-Term Results. J. Endod. 2014, 40, 1746-1751. [CrossRef] [PubMed]

18. Parirokh, M.; Torabinejad, M.; Dummer, P.M.H. Mineral Trioxide Aggregate and Other Bioactive Endodontic Cements: An Updated Overview_Part I: Vital Pulp Therapy. Int. Endod. J. 2018, 51, 177-205. [CrossRef] [PubMed]

19. Parirokh, M.; Torabinejad, M. Mineral Trioxide Aggregate: A Comprehensive Literature Review-Part Iii: Clinical Applications, Drawbacks, and Mechanism of Action. J. Endod. 2010, 36, 400-413. [CrossRef] [PubMed]

20. Camilleri, J.; Laurent, P.; About, I. Hydration of Biodentine, Theracal Lc, and a Prototype Tricalcium Silicate-Based Dentin Replacement Material after Pulp Capping in Entire Tooth Cultures. J. Endod. 2014, 40, 1846-1854. [CrossRef] [PubMed]

21. Gandolfi, M.G.; Siboni, F.; Prati, C. Chemical-Physical Properties of Theracal, a Novel Light-Curable Mta-Like Material for Pulp Capping. Int. Endod. J. 2012, 45, 571-579. [CrossRef] [PubMed] 
22. Sauro, S.; Babbar, A.; Gharibi, B.; Feitosa, V.P.; Carvalho, R.M.; Rodrigues, L.K.A.; Banerjee, A.; Watson, T. Cellular Differentiation, Bioactive and Mechanical Properties of Experimental Light-Curing Pulp Protection Materials. Dent. Mater. Off. Publ. Acad. Dent. Mate. 2018, 34, 868-878. [CrossRef]

23. Rodrigues, S.B.; Collares, F.M.; Leitune, V.C.; Schneider, L.F.; Ogliari, F.A.; Petzhold, C.L.; Samuel, S.M. Influence of Hydroxyethyl Acrylamide Addition to Dental Adhesive Resin. Dent. Mater. 2015, 31, 1579-1586. [CrossRef]

24. Rodrigues, S.B.; Petzhold, C.L.; Gamba, D.; Leitune, V.C.B.; Collares, F.M. Acrylamides and Methacrylamides as Alternative Monomers for Dental Adhesives. Dent. Mater. 2018, 34, 1634-1644. [CrossRef]

25. Yoshihara, K.; Nagaoka, N.; Okihara, T.; Irie, M.; Matsukawa, A.; Pedano, M.S.; Maruo, Y.; Yoshida, Y.; van Meerbeek, B. Development of Self-Adhesive Pulp-Capping Agents Containing a Novel Hydrophilic and Highly Polymerizable Acrylamide Monomer. J. Mater. Chem. B 2000. [CrossRef]

26. Meraji, N.; Camilleri, J. Bonding over Dentin Replacement Materials. J. Endod. 2017, 43, 1343-1349. [CrossRef]

27. Giraud, T.; Jeanneau, C.; Bergmann, M.; Laurent, P.; About, I. Tricalcium Silicate Capping Materials Modulate Pulp Healing and Inflammatory Activity in Vitro. J. Endod. 2018, 44, 1686-1691. [CrossRef]

28. Jeanneau, C.; Laurent, P.; Rombouts, C.; Giraud, T.; About, I. Light-Cured Tricalcium Silicate Toxicity to the Dental Pulp. J. Endod. 2017, 43, 2074-2080. [CrossRef]

29. Schertl, P.; Volk, J.; Perduns, R.; Adam, K.; Leyhausen, G.; Bakopoulou, A.; Geurtsen, W. Impaired Angiogenic Differentiation of Dental Pulp Stem Cells During Exposure to the Resinous Monomer Triethylene Glycol Dimethacrylate. Dent. Mater. 2019, 35, 144-155. [CrossRef]

30. Yu, L.; Katherine, D.; Lin, L. Polymer Blends and Composites from Renewable Resources. Prog. Polym. Sci. 2006, 31, 576-602. [CrossRef]

31. Pillai, C.K.S. Challenges for Natural Monomers and Polymers: Novel Design Strategies and Engineering to Develop Advanced Polymers. Des. Monomers Polym. 2010, 13, 87-121. [CrossRef]

32. Poulopoulou, N.; Smyrnioti, D.; Nikolaidis, G.N.; Tsitsimaka, I.; Christodoulou, E.; Bikiaris, D.N.; Charitopoulou, M.A.; Achilias, D.S.; Kapnisti, M.; Papageorgiou, G.Z. Sustainable Plastics from Biomass: Blends of Polyesters Based on 2,5-Furandicarboxylic Acid. Polymers 2020, 12, 225. [CrossRef]

33. Steven-Friso, K.; Ruijten, D.; Trullemans, L.; Renders, T.; van Puyvelde, P.; Witters, H.; Sels, B.F. Regioselective Synthesis, Isomerisation, in Vitro Oestrogenic Activity, and Copolymerisation of Bisguaiacol F (Bgf) Isomers. Green Chem. 2019, 21, 6622-6633.

34. Zamboulis, A.; Nakiou, E.A.; Christodoulou, E.; Bikiaris, D.N.; Kontonasaki, E.; Liverani, L.; Boccaccini, A.R. Polyglycerol Hyperbranched Polyesters: Synthesis, Properties and Pharmaceutical and Biomedical Applications. Int. J. Mol. Sci. 2019, 20, 6210. [CrossRef]

35. Park, J.H.; Gillispie, G.J.; Copus, J.S.; Zhang, W.; Atala, A.; Yoo, J.J.; Yelick, P.C.; Lee, S.J. The Effect of Bmp-Mimetic Peptide Tethering Bioinks on the Differentiation of Dental Pulp Stem Cells (Dpscs) in 3d Bioprinted Dental Constructs. Biofabrication 2020. [CrossRef]

36. Lizarraga Valderrama, L.D.R.; Nigmatullin, R.; Ladino, B.; Taylor, C.S.; Boccaccini, A.R.; Knowles, J.C.; Claeyssens, F.; Haycock, J.W.; Roy, I. Modulation of Neuronal Cell Affinity of Composites Scaffolds Based on Polyhydroxyalkanoates and Bioactive Glasses. Biomed. Mater. 2020. [CrossRef]

37. Okamoto, M.; Matsumoto, S.; Sugiyama, A.; Kanie, K.; Watanabe, M.; Huang, H.; Ali, M.; Ito, Y.; Miura, J.; Hirose, Y.; et al. Performance of a Biodegradable Composite with Hydroxyapatite as a Scaffold in Pulp Tissue Repair. Polymers 2020, 12, 937. [CrossRef]

38. Asgary, S.; Eghbal, M.J. Treatment Outcomes of Pulpotomy in Permanent Molars with Irreversible Pulpitis Using Biomaterials: A Multi-Center Randomized Controlled Trial. Acta Odontol. Scand. 2013, 71, 130-136. [CrossRef]

39. Eghbal, M.J.; Asgary, S.; Baglue, R.A.; Parirokh, M.; Ghoddusi, J. Mta Pulpotomy of Human Permanent Molars with Irreversible Pulpitis. Aust. Endod. J. 2009, 35, 4-8. [CrossRef] [PubMed]

40. Asgary, S.; Eghbal, M.J.; Fazlyab, M.; Baghban, A.A.; Ghoddusi, J. Five-Year Results of Vital Pulp Therapy in Permanent Molars with Irreversible Pulpitis: A Non-Inferiority Multicenter Randomized Clinical Trial. Clin. Oral Investig. 2015, 19, 335-341. [CrossRef]

41. Taha, N.A.; Ahmad, M.B.; Ghanim, A. Assessment of Mineral Trioxide Aggregate Pulpotomy in Mature Permanent Teeth with Carious Exposures. Int. Endod. J. 2017, 50, 117-125. [CrossRef]

42. Caliskan, M.K. Pulpotomy of Carious Vital Teeth with Periapical Involvement. Int. Endod. J. 1995, 28, 172-176. [CrossRef] [PubMed] 
43. Asgary, S. Calcium-Enriched Mixture Pulpotomy of a Human Permanent Molar with Irreversible Pulpitis and Condensing Apical Periodontitis. J. Conserv. Dent. 2011, 14, 90-93. [CrossRef]

44. Asgary, S.; Eghbal, M.J.; Bagheban, A.A. Long-Term Outcomes of Pulpotomy in Permanent Teeth with Irreversible Pulpitis: A Multi-Center Randomized Controlled Trial. Am. J. Dent. 2017, 30, 151-155. [PubMed]

45. Ricucci, D.; Siqueira, J.F., Jr.; Li, Y.; Tay, F.R. Vital Pulp Therapy: Histopathology and Histobacteriology-Based Guidelines to Treat Teeth with Deep Caries and Pulp Exposure. J. Dent. 2019, 86, 41-52. [CrossRef]

46. Schwendicke, F.; Brouwer, F.; Schwendicke, A.; Paris, S. Different Materials for Direct Pulp Capping: Systematic Review and Meta-Analysis and Trial Sequential Analysis. Clin. Oral Investig. 2016, 20, 1121-1132. [CrossRef]

47. Sangwan, P.; Sangwan, A.; Duhan, J.; Rohilla, A. Tertiary Dentinogenesis with Calcium Hydroxide: A Review of Proposed Mechanisms. Int. Endod. J. 2013, 46, 3-19. [CrossRef]

48. Emara, R.; Elhennawy, K.; Schwendicke, F. Effects of Calcium Silicate Cements on Dental Pulp Cells: A Systematic Review. J. Dent. 2018, 77, 18-36. [CrossRef]

49. Paula, A.B.; Laranjo, M.; Marto, C.M.; Paulo, S.; Abrantes, A.M.; Casalta-Lopes, J.; Marques-Ferreira, M.; Botelho, M.F.; Carrilho, E. Direct Pulp Capping: What Is the Most Effective Therapy?-Systematic Review and Meta-Analysis. J. Evid. Based. Dent. Pract. 2018, 18, 298-314. [CrossRef]

50. Li, Z.; Cao, L.; Fan, M.; Xu, Q. Direct Pulp Capping with Calcium Hydroxide or Mineral Trioxide Aggregate: A Meta-Analysis. J. Endod. 2015, 41, 1412-1417. [CrossRef]

51. Sanz, J.L.; Forner, L.; Almudéver, A.; Guerrero-Gironés, J.; Llena, C. Viability and Stimulation of Human Stem Cells from the Apical Papilla (Hscaps) Induced by Silicate-Based Materials for Their Potential Use in Regenerative Endodontics: A Systematic Review. Materials 2020, 13, 974. [CrossRef]

52. Accorinte, M.L.R.; Loguercio, A.D.; Reis, A.; Carneiro, E.; Grande, R.H.M.; Murata, S.S.; Holland, R. Response of Human Dental Pulp Capped with Mta and Calcium Hydroxide Powder. Oper Dent. 2008, 33, 488-495. [CrossRef] [PubMed]

53. Accorinte, M.D.R.; Holland, R.; Reis, A.; Bortoluzzi, M.C.; Murata, S.S.; Dezan, E.; Souza, V.; Alessandro, L.D. Evaluation of Mineral Trioxide Aggregate and Calcium Hydroxide Cement as Pulp-Capping Agents in Human Teeth. J. Endod. 2008, 34, 1-6. [CrossRef] [PubMed]

54. Liberati, A.; Altman, D.G.; Tetzlaff, J.; Mulrow, C.; Gotzsche, P.C.; Ioannidis, J.P.; Clarke, M.; Devereaux, P.J.; Kleijnen, J.; Moher, D. The Prisma Statement for Reporting Systematic Reviews and Meta-Analyses of Studies That Evaluate Health Care Interventions: Explanation and Elaboration. Ann. Intern. Med. 2009, 151, W65-W94. [CrossRef] [PubMed]

55. Hutton, B.; Salanti, G.; Caldwell, D.M.; Chaimani, A.; Schmid, C.H.; Cameron, C.; Ioannidis, J.P.; Straus, S.; Thorlund, K.; Jansen, J.P.; et al. The Prisma Extension Statement for Reporting of Systematic Reviews Incorporating Network Meta-Analyses of Health Care Interventions: Checklist and Explanations. Ann. Intern. Med. 2015, 162, 777-784. [CrossRef] [PubMed]

56. Alliotlicht, B.; Jean, A.; Gregoire, M. Comparative Effect of Calcium Hydroxide and Hydroxyapatite on the Cellular-Activity of Human Pulp Fibroblasts in-Vitro. Arch. Oral Biol. 1994, 39, 481-489. [CrossRef]

57. Min, K.S.; Chang, H.S.; Bae, J.M.; Park, S.H.; Hong, C.U.; Kim, E.C. The Induction of Heme Oxygenase-1 Modulates Bismuth Oxide-Induced Cytotoxicity in Human Dental Pulp Cells. J. Endod. 2007, 33, 1342-1346. [CrossRef]

58. Min, K.S.; Kim, H.I.; Park, H.J.; Pi, S.H.; Hong, C.U.; Kim, E.C. Human Pulp Cells Response to Portland Cement in Vitro. J. Endod. 2007, 33, 163-166. [CrossRef]

59. Laurent, P.; Camps, J.; de Meo, M.; Dejou, J.; About, I. Induction of Specific Cell Responses to a $\mathrm{Ca}$ (3)Sio(5)-Based Posterior Restorative Material. Dent. Mater. 2008, 24, 1486-1494. [CrossRef]

60. Min, K.S.; Lee, S.I.; Lee, Y.; Kim, E.C. Effect of Radiopaque Portland Cement on Mineralization in Human Dental Pulp Cells. Oral Surg. Oral Med. Oral Pathol. Oral Radiol. Endod. 2009, 108, e82-e86. [CrossRef]

61. Lee, J.B.; Park, S.J.; Kim, H.H.; Kwon, Y.S.; Lee, K.W.; Min, K.S. Physical Properties and Biological/Odontogenic Effects of an Experimentally Developed Fast-Setting Alpha-Tricalcium Phosphate-Based Pulp Capping Material. BMC Oral Health 2014, 14, 87. [CrossRef]

62. Bortoluzzi, E.A.; Niu, L.N.; Palani, C.D.; El-Awady, A.R.; Hammond, B.D.; Pei, D.D.; Tian, F.C.; Cutler, C.W.; Pashley, D.H.; Tay, F.R. Cytotoxicity and Osteogenic Potential of Silicate Calcium Cements as Potential Protective Materials for Pulpal Revascularization. Dent. Mater. 2015, 31, 1510-1522. [CrossRef] [PubMed] 
63. Niu, L.N.; Watson, D.; Thames, K.; Primus, C.M.; Bergeron, B.E.; Jiao, K.; Bortoluzzi, E.A.; Cutler, C.W.; Chen, J.H.; Pashley, D.H.; et al. Effects of a Discoloration-Resistant Calcium Aluminosilicate Cement on the Viability and Proliferation of Undifferentiated Human Dental Pulp Stem Cells. Sci. Rep. 2015, 5, 17177. [CrossRef] [PubMed]

64. Oncel Torun, Z.; Torun, D.; Demirkaya, K.; Yavuz, S.T.; Elci, M.P.; Sarper, M.; Avcu, F. Effects of Iroot Bp and White Mineral Trioxide Aggregate on Cell Viability and the Expression of Genes Associated with Mineralization. Int. Endod. J. 2015, 48, 986-993. [CrossRef] [PubMed]

65. Zhang, J.; Zhu, L.X.; Cheng, X.; Lin, Y.; Yan, P.; Peng, B. Promotion of Dental Pulp Cell Migration and Pulp Repair by a Bioceramic Putty Involving Fgfr-Mediated Signaling Pathways. J. Dent. Res. 2015, 94, 853-862. [CrossRef] [PubMed]

66. Chung, C.J.; Kim, E.; Song, M.; Park, J.W.; Shin, S.J. Effects of Two Fast-Setting Calcium-Silicate Cements on Cell Viability and Angiogenic Factor Release in Human Pulp-Derived Cells. Odontology 2016, 104, $143-151$. [CrossRef] [PubMed]

67. Daltoe, M.O.; Paula-Silva, F.W.; Faccioli, L.H.; Gaton-Hernandez, P.M.; de Rossi, A.; Silva, L.A.B. Expression of Mineralization Markers During Pulp Response to Biodentine and Mineral Trioxide Aggregate. J. Endod. 2016, 42, 596-603. [CrossRef]

68. Widbiller, M.; Lindner, S.R.; Buchalla, W.; Eidt, A.; Hiller, K.A.; Schmalz, G.; Galler, K.M. Three-Dimensional Culture of Dental Pulp Stem Cells in Direct Contact to Tricalcium Silicate Cements. Clin. Oral Investig. 2016, 20, 237-246. [CrossRef]

69. Jun, S.K.; Lee, J.H.; Lee, H.H. The Biomineralization of a Bioactive Glass-Incorporated Light-Curable Pulp Capping Material Using Human Dental Pulp Stem Cells. Biomed Res. Int. 2017, 2017, 2495282. [CrossRef]

70. Lee, B.N.; Lee, B.G.; Chang, H.S.; Hwang, Y.C.; Hwang, I.N.; Oh, W.M. Effects of a Novel Light-Curable Material on Odontoblastic Differentiation of Human Dental Pulp Cells. Int. Endod. J. 2017, 50, 464-471. [CrossRef]

71. Mestieri, L.B.; Gomes-Cornelio, A.L.; Rodrigues, E.M.; Faria, G.M.; Guerreiro-Tanomaru, J.; Tanomaru-Filho, M. Cytotoxicity and Bioactivity of Calcium Silicate Cements Combined with Niobium Oxide in Different Cell Lines. Braz. Dent. J. 2017, 28, 65-71. [CrossRef]

72. Rodrigues, E.M.; Cornelio, A.L.G.; Mestieri, L.B.; Fuentes, A.S.C.; Salles, L.P.; Rossa-Junior, C.; Faria, G.; Guerreiro-Tanomaru, J.M.; Tanomaru-Filho, M. Human Dental Pulp Cells Response to Mineral Trioxide Aggregate (Mta) and Mta Plus: Cytotoxicity and Gene Expression Analysis. Int. Endod. J. 2017, 50, 780-789. [CrossRef]

73. Sun, Y.; Luo, T.; Shen, Y.; Haapasalo, M.; Zou, L.; Liu, J. Effect of Iroot Fast Set Root Repair Material on the Proliferation, Migration and Differentiation of Human Dental Pulp Stem Cells in Vitro. PLoS ONE 2017, 12, e0186848. [CrossRef] [PubMed]

74. Tomas-Catala, C.J.; Collado-Gonzalez, M.; Garcia-Bernal, D.; Onate-Sanchez, R.E.; Forner, L.; Llena, C.; Lozano, A.; Castelo-Baz, P.; Moraleda, J.M.; Rodriguez-Lozano, F.J. Comparative Analysis of the Biological Effects of the Endodontic Bioactive Cements Mta-Angelus, Mta Repair Hp and Neomta Plus on Human Dental Pulp Stem Cells. Int. Endod. J. 2017, 50 (Suppl. S2), e63-e72. [CrossRef]

75. Collado-Gonzalez, M.; Pecci-Lloret, M.R.; Tomas-Catala, C.J.; Garcia-Bernal, D.; Onate-Sanchez, R.E.; Llena, C.; Forner, L.; Rosa, V.; Rodriguez-Lozano, F.J. Thermo-Setting Glass Ionomer Cements Promote Variable Biological Responses of Human Dental Pulp Stem Cells. Dent. Mater. 2018, 34, 932-943. [CrossRef] [PubMed]

76. Pedano, M.S.; Li, X.; Li, S.; Sun, Z.; Cokic, S.M.; Putzeys, E.; Yoshihara, K.; Yoshida, Y.; Chen, Z.; van Landuyt, K.; et al. Freshly-Mixed and Setting Calcium-Silicate Cements Stimulate Human Dental Pulp Cells. Dent. Mater. 2018, 34, 797-808. [CrossRef]

77. Tomas-Catala, C.J.; Collado-Gonzalez, M.; Garcia-Bernal, D.; Onate-Sanchez, R.E.; Forner, L.; Llena, C.; Lozano, A.; Moraleda, J.M.; Rodriguez-Lozano, F.J. Biocompatibility of New Pulp-Capping Materials Neomta Plus, Mta Repair Hp, and Biodentine on Human Dental Pulp Stem Cells. J. Endod. 2018, 44, 126-132. [CrossRef] [PubMed]

78. Lee, S.M.; Kim, S.Y.; Kim, J.H.; Jun, S.K.; Kim, H.W.; Lee, J.H.; Lee, H.H. Depth-Dependent Cellular Response from Dental Bulk-Fill Resins in Human Dental Pulp Stem Cells. Stem Cells Int. 2019, 2019, 1251536. [CrossRef] [PubMed] 
79. Lopez-Garcia, S.; Pecci-Lloret, M.P.; Pecci-Lloret, M.R.; Onate-Sanchez, R.E.; Garcia-Bernal, D.; Castelo-Baz, P.; Rodriguez-Lozano, F.J.; Guerrero-Girones, J. In Vitro Evaluation of the Biological Effects of Activa Kids Bioactive Restorative, Ionolux, and Riva Light Cure on Human Dental Pulp Stem Cells. Materials 2019, 12, 3694. [CrossRef]

80. Dou, L.; Yan, Q.; Yang, D. Effect of Five Dental Pulp Capping Agents on Cell Proliferation, Viability, Apoptosis and Mineralization of Human Dental Pulp Cells. Exp. Ther. Med. 2020, 19, 2377-2383. [CrossRef]

81. Hebling, J.; Giro, E.M.A.; Costa, C.A.D. Biocompatibility of an Adhesive System Applied to Exposed Human Dental Pulp. J. Endod. 1999, 25, 676-682. [CrossRef]

82. Do Nascimento, A.B.L.; Fontana, U.F.; Teixeira, H.M.; Costa, C.A.D. Biocompatibility of a Resin-Modified Glass-Ionomer Cement Applied as Pulp Capping in Human Teeth. Am. J. Dent. 2000, 13, 28-34. [PubMed]

83. Costa, C.A.D.; Nascimento, A.B.L.d.; Teixeira, H.M.; Fontana, U.F. Response of Human Pulps Capped with a Self-Etching Adhesive System. Dent. Mater. 2001, 17, 230-240. [CrossRef]

84. Ersin, N.K.; Eronat, N. The Comparison of a Dentin Adhesive with Calcium Hydroxide as a Pulp-Capping Agent on the Exposed Pulps of Human and Sheep Teeth. Quintessence Int. 2005, 36, 271-280.

85. Olsson, H.; Davies, J.R.; Holst, K.E.; Schroder, U.; Petersson, K. Dental Pulp Capping: Effect of Emdogain Gel on Experimentally Exposed Human Pulps. Int. Endod. J. 2005, 38, 186-194. [CrossRef] [PubMed]

86. Silva, G.A.B.; Lanza, L.D.; Lopes, N., Jr.; Moreira, A.; Alves, J.B. Direct Pulp Capping with a Dentin Bonding System in Human Teeth: A Clinical and Histological Evaluation. Oper. Dent. 2006, 31, 297-307. [CrossRef] [PubMed]

87. Sawicki, L.; Pameijer, C.H.; Emerich, K.; Adamowicz-Klepalska, B. Histological Evaluation of Mineral Trioxide Aggregate and Calcium Hydroxide in Direct Pulp Capping of Human Immature Permanent Teeth. Am. J. Dent. 2008, 21, 262-266. [PubMed]

88. Azimi, S.; Fazlyab, M.; Sadri, D.; Saghiri, M.A.; Khosravanifard, B.; Asgary, S. Comparison of Pulp Response to Mineral Trioxide Aggregate and a Bioceramic Paste in Partial Pulpotomy of Sound Human Premolars: A Randomized Controlled Trial. Int. Endod. J. 2014, 47, 873-881. [CrossRef]

89. Swarup, S.J.; Rao, A.; Boaz, K.; Srikant, N.; Shenoy, R. Pulpal Response to Nano Hydroxyapatite, Mineral Trioxide Aggregate and Calcium Hydroxide When Used as a Direct Pulp Capping Agent: An in Vivo Study. J. Clin. Pediatr. Dent. 2014, 38, 201-206. [CrossRef]

90. Banava, S.; Fazlyab, M.; Heshmat, H.; Mojtahedzadeh, F.; Motahhary, P. Histological Evaluation of Single and Double-Visit Direct Pulp Capping with Different Materials on Sound Human Premolars: A Randomized Controlled Clinical Trial. Iran. Endod. J. 2015, 10, 82-88.

91. Demarco, F.F.; Tarquinio, S.B.; Jaeger, M.M.; de Araújo, V.C.; Matson, E. Pulp Response and Cytotoxicity Evaluation of 2 Dentin Bonding Agents. Quintessence Int. (Berlin, Germany: 1985) 2001, 32, 211-220.

92. Accorinte Mde, L.; Loguercio, A.D.; Reis, A.; Muench, A.; de Araujo, V.C. Adverse Effects of Human Pulps after Direct Pulp Capping with the Different Components from a Total-Etch, Three-Step Adhesive System. Dent. Mater. 2005, 21, 599-607. [CrossRef] [PubMed]

93. Accorinte, M.D.L.R.; Reis, A.; Loguercio, A.D.; de Araújo, V.C.; Muench, A. Influence of Rubber Dam Isolation on Human Pulp Responses after Capping with Calcium Hydroxide and an Adhesive System. Quintessence Int. 2006, 37, 205-212.

94. Iwamoto, C.E.; Adachi, E.; Pameijer, C.H.; Barnes, D.; Romberg, E.E.; Jefferies, S. Clinical and Histological Evaluation of White Proroot Mta in Direct Pulp Capping. Am. J. Dent. 2006, 19, 85-90. [PubMed]

95. Accorinte, M.D.R.; Loguercio, A.D.; Reis, A.; Holland, R. Effects of Hemostatic Agents on the Histomorphologic Response of Human Dental Pulp Capped with Calcium Hydroxide. Quintessence Int. 2007, 38, 843-852.

96. Elias, R.V.; Demarco, F.F.; Tarquinio, S.B.; Piva, E. Pulp Responses to the Application of a Self-Etching Adhesive in Human Pulps after Controlling Bleeding with Sodium Hypochlorite. Quintessence Int. 2007, 38, e67-e77. [PubMed]

97. Accorinte, M.L.R.; Loguercio, A.D.; Reis, A.; Costa, C.A.S. Response of Human Pulps Capped with Different Self-Etch Adhesive Systems. Clin. Oral Investig. 2008, 12, 119-127. [CrossRef]

98. Lu, Y.; Liu, T.; Li, H.; Pi, G. Histological Evaluation of Direct Pulp Capping with a Self-Etching Adhesive and Calcium Hydroxide on Human Pulp Tissue. Int. Endod. J. 2008, 41, 643-650. [CrossRef] 
99. Min, K.S.; Park, H.J.; Lee, S.K.; Park, S.H.; Hong, C.U.; Kim, H.W.; Lee, H.H.; Kim, E.C. Effect of Mineral Trioxide Aggregate on Dentin Bridge Formation and Expression of Dentin Sialoprotein and Heme Oxygenase-1 in Human Dental Pulp. J. Endod. 2008, 34, 666-670. [CrossRef]

100. Nair, P.N.R.; Duncan, H.F.; Ford, T.R.P.; Luder, H.U. Histological, Ultrastructural and Quantitative Investigations on the Response of Healthy Human Pulps to Experimental Capping with Mineral Trioxide Aggregate: A Randomized Controlled Trial. Int. Endod. J. 2008, 41, 128-150.

101. Accorinte, M.L.; Loguercio, A.D.; Reis, A.; Bauer, J.R.; Grande, R.H.; Murata, S.S.; Souza, V.; Holland, R. Evaluation of Two Mineral Trioxide Aggregate Compounds as Pulp-Capping Agents in Human Teeth. Int. Endod. J. 2009, 42, 122-128. [CrossRef]

102. Kiatwateeratana, T.; Kintarak, S.; Piwat, S.; Chankanka, O.; Kamaolmatyakul, S.; Thearmontree, A. Partial Pulpotomy on Caries-Free Teeth Using Enamel Matrix Derivative or Calcium Hydroxide: A Randomized Controlled Trial. Int. Endod. J. 2009, 42, 584-592. [CrossRef] [PubMed]

103. Parolia, A.; Kundabala, M.; Rao, N.N.; Acharya, S.R.; Agrawal, P.; Mohan, M.; Thomas, M. A Comparative Histological Analysis of Human Pulp Following Direct Pulp Capping with Propolis, Mineral Trioxide Aggregate and Dycal. Aust. Dent. J. 2010, 55, 59-64. [CrossRef] [PubMed]

104. Nowicka, A.; Lipski, M.; Parafiniuk, M.; Sporniak-Tutak, K.; Lichota, D.; Kosierkiewicz, A.; Kaczmarek, W.; Buczkowska-Radlinska, J. Response of Human Dental Pulp Capped with Biodentine and Mineral Trioxide Aggregate. J. Endod. 2013, 39, 743-747. [CrossRef] [PubMed]

105. Nowicka, A.; Lagocka, R.; Lipski, M.; Parafiniuk, M.; Grocholewicz, K.; Sobolewska, E.; Witek, A.; Buczkowska-Radlinska, J. Clinical and Histological Evaluation of Direct Pulp Capping on Human Pulp Tissue Using a Dentin Adhesive System. Biomed Res. Int. 2016, 2016, 2591273. [CrossRef]

106. Bakhtiar, H.; Nekoofar, M.H.; Aminishakib, P.; Abedi, F.; Moosavi, F.N.; Esnaashari, E.; Azizi, A.; Esmailian, S.; Ellini, M.R.; Mesgarzadeh, V.; et al. Human Pulp Responses to Partial Pulpotomy Treatment with Theracal as Compared with Biodentine and Proroot Mta: A Clinical Trial. J. Endod. 2017, 43, 1786-1791. [CrossRef]

107. Jalan, A.L.; Warhadpande, M.M.; Dakshindas, D.M. A Comparison of Human Dental Pulp Response to Calcium Hydroxide and Biodentine as Direct Pulp-Capping Agents. J. Conserv. Dent. 2017, 20, 129-133. [CrossRef]

108. Mehrvarzfar, P.; Abbott, P.V.; Mashhadiabbas, F.; Vatanpour, M.; Savadkouhi, S.T. Clinical and Histological Responses of Human Dental Pulp to Mta and Combined Mta/Treated Dentin Matrix in Partial Pulpotomy. Aust. Endod. J. 2018, 44, 46-53. [CrossRef]

109. Higgins, J.P.T.; Thomas, J.; Chandler, J.; Cumpston, M.; Li, T.; Page, M.J.; Welch, V.A. (Eds.) Cochrane Handbook for Systematic Reviews of Interventions Version. 6.0.; Cochrane: London, UK, 2019.

110. The Cochrane Collaboration. Review Manager (Revman) [Computer Program]. Version 5.3.; The Nordic Cochrane Centre: Copenhagen, Denmark, 2014.

111. Guyatt, G.H.; Oxman, A.D.; Kunz, R.; Vist, G.E.; Falck-Ytter, Y.; Schünemann, H.J. What Is Quality of Evidence and Why Is It Important to Clinicians? BMJ 2008, 336, 995-998. [CrossRef] [PubMed]

112. Accorinte Mde, L.; Loguercio, A.D.; Reis, A.; Muench, A.; de Araujo, V.C. Response of Human Pulp Capped with a Bonding Agent after Bleeding Control with Hemostatic Agents. Oper. Dent. 2005, 30, 147-155. [PubMed]

113. Fransson, H.; Petersson, K.; Davies, J.R. Dentine Sialoprotein and Collagen I Expression after Experimental Pulp Capping in Humans Using Emdogain (R) Gel. Int. Endod. J. 2011, 44, 259-267. [CrossRef] [PubMed]

114. Madani, Z.S.; Haddadi, A.; Mesgarani, A.; Seyedmajidi, M.; Mostafazadeh, A.; Bijani, A.; Ashraphpour, M. Histopathologic Responses of the Dental Pulp to Calcium-Enriched Mixture (Cem) and Mineral Trioxide Aggregate (Mta) in Diabetic and Non-Diabetic Rats. Int. J. Mol. Cell. Med. 2014, 3, 263-271. [PubMed]

115. Leite, M.F.; Ganzerla, E.; Marques, M.M.; Nicolau, J. Diabetes Induces Metabolic Alterations in Dental Pulp. J. Endod. 2008, 34, 1211-1214. [CrossRef]

116. Kakehashi, S.; Stanley, H.R.; Fitzgerald, R.J. The Effects of Surgical Exposures of Dental Pulps in Germ-Free and Conventional Laboratory Rats. Oral Surg. Oral Med. Oral Pathol. 1965, 20, 340-349. [CrossRef]

117. Nyborg, H. Healing Processes in the Pulp on Capping; a Morphologic Study; Experiments on Surgical Lesions of the Pulp in Dog and Man. Acta Odontol. Scand. 1955, 13 (Suppl. S16), 1-130.

118. Martignoni, M.; Groothuis, G.M.; de Kanter, R. Species Differences between Mouse, Rat, Dog, Monkey and Human Cyp-Mediated Drug Metabolism, Inhibition and Induction. Expert Opin. Drug Metab. Toxicol. 2006, 2, 875-894. [CrossRef] [PubMed] 
119. Ricucci, D.; Siqueira, J.F.; Ricucci, D.; Siqueira, J.F., Jr. Pulp Response to Caries and Restorative Procedures. In Endodontology: An Integrated Biological and Clinical View; Edizioni Martina: Bologna, Italy, 2013.

120. Schweikl, H.; Spagnuolo, G.; Schmalz, G. Genetic and Cellular Toxicology of Dental Resin Monomers. J. Dent. Res. 2006, 85, 870-877. [CrossRef] [PubMed]

121. Caldas, I.P.; Alves, G.G.; Barbosa, I.B.; Scelza, P.; de Noronha, F.; Scelza, M.Z. In Vitro Cytotoxicity of Dental Adhesives: A Systematic Review. Dent. Mater. 2019, 35, 195-205. [CrossRef] [PubMed]

122. Van Landuyt, K.L.; Krifka, S.; Hiller, K.A.; Bolay, C.; Waha, C.; van Meerbeek, B.; Schmalz, G.; Schweikl, H. Evaluation of Cell Responses toward Adhesives with Different Photoinitiating Systems. Dent. Mater. 2015, 31, 916-927. [CrossRef]

123. Schneider, T.R.; Hakami-Tafreshi, R.; Tomasino-Perez, A.; Tayebi, L.; Lobner, D. Effects of Dental Composite Resin Monomers on Dental Pulp Cells. Dent. Mater. J. 2019, 38, 579-583. [CrossRef]

124. Costa, C.A.D.; Teixeira, H.M.; Nascimento, A.B.L.d.; Hebling, J. Biocompatibility of an Adhesive System and 2-Hydroxyethylmethacrylate. J. Dent. Child. 1999, 66, 337-342.

125. Teixeira, H.M.; Nascimento, A.B.L.D.; Hebling, J.; Costa, C.A.D. In Vivo Evaluation of the Biocompatibility of Three Current Bonding Agents. J. Oral Rehabil. 2006, 33, 542-550. [CrossRef] [PubMed]

126. Ricucci, D. Patologia E Clinica Endodontica; Edizione Martina: Bologna, Italy, 2009.

127. Bjorndal, L.; Reit, C.; Bruun, G.; Markvart, M.; Kjaeldgaard, M.; Nasman, P.; Thordrup, M.; Dige, I.; Nyvad, B.; Fransson, H.; et al. Treatment of Deep Caries Lesions in Adults: Randomized Clinical Trials Comparing Stepwise Vs. Direct Complete Excavation, and Direct Pulp Capping Vs. Partial Pulpotomy. Eur. J. Oral Sci. 2010, 118, 290-297. [CrossRef] [PubMed]

128. Barthel, C.R.; Rosenkranz, B.; Leuenberg, A.; Roulet, J.F. Pulp Capping of Carious Exposures: Treatment Outcome after 5 and 10 Years: A Retrospective Study. J. Endod. 2000, 26, 525-528. [CrossRef]

129. Holland, R.; de Souza, V.; de Mello, W.; Nery, M.J.; Bernabe, P.F.; Filho, J.A.O. Healing Process after Pulpotomy and Covering with Calcium Hydroxide, Dycal or Mpc. Histological Study in Dog Teeth. Rev. Fac. Odontol. Aracatuba 1978, 7, 185-191. [PubMed]

130. Holland, R.; de Mello, W.; Nery, M.J.; de Souza, V.; Bernabe, P.F.; Filho, J.A.O. Healing Process of Dog Dental Pulp after Pulpotomy and Pulp Covering with Calcium Hydroxide in Powder or Paste Form. Acta Odontol. Pediatr. 1981, 2, 47-51. [PubMed]

131. Nowicka, A.; Wilk, G.; Lipski, M.; Kolecki, J.; Buczkowska-Radlinska, J. Tomographic Evaluation of Reparative Dentin Formation after Direct Pulp Capping with $\mathrm{Ca}(\mathrm{Oh}) 2$, Mta, Biodentine, and Dentin Bonding System in Human Teeth. J. Endod. 2015, 41, 1234-1240. [CrossRef]

132. Glass, R.L.; Zander, H.A. Pulp Healing. J. Dent. Res. 1949, 28, 97-107. [CrossRef] [PubMed]

133. Dammaschke, T. The History of Direct Pulp Capping. J. Hist. Dent. 2008, 56, 9-23. [PubMed]

134. Shovelton, D.S.; Friend, L.A.; Kirk, E.E.; Rowe, A.H. The Efficacy of Pulp Capping Materials. A Comparative Trial. Br. Dent. J. 1971, 130, 385-391. [CrossRef]

135. Sanz, J.L.; Rodríguez-Lozano, F.J.; Llena, C.; Sauro, S.; Forner, L. Bioactivity of Bioceramic Materials Used in the Dentin-Pulp Complex Therapy: A Systematic Review. Materials 2019, 12, 1015. [CrossRef]

136. Urquhart, O.; Tampi, M.P.; Pilcher, L.; Slayton, R.L.; Araujo, M.W.B.; Fontana, M.; Guzmán-Armstrong, S.; Nascimento, M.M.; Nový, B.B.; Tinanoff, N.; et al. Nonrestorative Treatments for Caries: Systematic Review and Network Meta-Analysis. J. Dent. Res. 2019, 98, 14-26. [CrossRef] [PubMed]

137. Briso, A.L.F.; Rahal, V.; Mestrener, S.R.; Dezan, E., Jr. Biological Response of Pulps Submitted to Different Capping Materials. Braz. Oral Res. 2006, 20, 219-225. [CrossRef] [PubMed]

138. Gronthos, S.; Mankani, M.; Brahim, J.; Robey, P.G.; Shi, S. Postnatal Human Dental Pulp Stem Cells (Dpscs) in Vitro and in Vivo. Proc. Natl. Acad. Sci. USA 2000, 97, 13625-13630. [CrossRef]

139. About, I.; Bottero, M.J.; de Denato, P.; Camps, J.; Franquin, J.C.; Mitsiadis, T.A. Human Dentin Production in Vitro. Exp. Cell Res. 2000, 258, 33-41. [CrossRef] [PubMed]

140. About, I.; Laurent-Maquin, D.; Lendahl, U.; Mitsiadis, T.A. Nestin Expression in Embryonic and Adult Human Teeth under Normal and Pathological Conditions. Am. J. Pathol. 2000, 157, 287-295. [CrossRef]

(C) 2020 by the authors. Licensee MDPI, Basel, Switzerland. This article is an open access article distributed under the terms and conditions of the Creative Commons Attribution (CC BY) license (http://creativecommons.org/licenses/by/4.0/). 